JOURNAL OF THE

AMERICAN MATHEMATICAL SOCIETY

Volume 9, Number 3, July 1996

\title{
SW $\Rightarrow$ Gr: FROM THE SEIBERG-WITTEN EQUATIONS TO PSEUDO-HOLOMORPHIC CURVES
}

\author{
CLIFFORD H. TAUBES
}

\section{INTRODUCTION}

The purpose of this article is to explain how pseudo-holomorphic curves in a symplectic 4-manifold can be constructed from solutions to the Seiberg-Witten equations. As such, the main theorem proved here (Theorem 1.3) is an existence theorem for pseudo-holomorphic curves. This article thus provides a proof of roughly half of the main theorem in the announcement [T1]. That theorem, Theorem 4.1, asserts an equivalence between the Seiberg-Witten invariants for a symplectic manifold and a certain Gromov invariant which counts (with signs) the number of pseudoholomorphic curves in a given homology class.

The Seiberg-Witten invariants were introduced to mathematicians by Witten [W] based on his joint work with Nat Seiberg [SW1], [SW2]. A description of these invariants is given in Section 1. (See also [KM1], [T1].) Suffice it to say here that when $X$ is a compact, oriented, 4-dimensional manifold with

$$
b_{2}^{+} \equiv \frac{1}{2}\left(\operatorname{rank}\left(H^{2}(X ; \mathbb{R})\right)+\text { signature }\right)
$$

at least 2 , then these invariants define a diffeomorphism invariant map, SW, from the set of equivalence classes, Spin, of $\operatorname{Spin}^{\mathbb{C}}$ structures on $X$ to $\mathbb{Z}$. Note that the set Spin has naturally the structure of a principal $H^{2}(X ; \mathbb{Z})$ bundle over a point.

A symplectic 4-manifold is a pair $(X, \omega)$, where $X$ is a 4-manifold and where $\omega$ is a closed 2-form with $\omega \wedge \omega$ nowhere zero. Thus, a symplectic 4-manifold has a canonical orientation. A symplectic 4-manifold also has a complex line bundle, $K$, (called the canonical bundle) which is canonical up to isomorphism. And, as explained in [T1] or [T2], a symplectic 4-manifold has a canonical equivalence class of Spin ${ }^{\mathbb{C}}$ structure. The latter endows Spin with a base point and so gives the identification

$$
\operatorname{Spin} \approx H^{2}(X ; \mathbb{Z}) \text {. }
$$

(Both the identification of Spin and the choice of orientation do not change under a continuous deformation of the symplectic form.) In the ensuing discussion, the canonical orientation for a symplectic manifold and the identification in (0.2) will be assumed implicitly. Thus, with (0.2) understood, SW defines a map

$$
\mathrm{SW}: H^{2}(X ; \mathbb{Z}) \rightarrow \mathbb{Z} \text {. }
$$

Received by the editors June 26, 1995 and, in revised form, August 7, 1995.

1991 Mathematics Subject Classification. Primary 53C07, 53C15.

The author is supported in part by the National Science Foundation.

(C)1996 American Mathematical Society 
(Note that SW as a map from Spin is diffeomorphism invariant, but that the identification in (0.2) is not. The effect of a diffeomorphism on (0.3) depends on the behavior of $c_{1}(K)$.)

A submanifold $\Sigma$ of a symplectic manifold is called symplectic when the restriction of the symplectic form to $T \Sigma$ is non-degenerate.

With the preceding understood, the following is a corollary to Theorem 1.3 (with Proposition 7.1):

Theorem 0.1. Let $X$ be a compact, oriented, 4-dimensional symplectic manifold with $b_{2}^{+}>1$. Let $e \neq 0 \in H^{2}(X ; \mathbb{Z})$ be a class with $\mathrm{SW}(e) \neq 0$. Then the Poincaré dual to $e$ is represented by the fundamental class of an embedded, symplectic curve. And, this curve has genus $g=1+e \cdot e$.

In the preceding, · denotes the cup product pairing from $H^{2}(X ; \mathbb{Z})$ to $\mathbb{Z}$. Thus, the number $e \cdot e$ is the value of the cup of $e$ with itself on $X$ 's fundamental class.

(Remark that Simon Donaldson [Do] announced last year a theorem to the effect that classes $e \in H_{2}(X ; \mathbb{Z})$ with very large positive pairing with $\omega$ have embedded, symplectic representatives.)

Various other consequences of Theorem 1.3 and Proposition 7.1 were discussed in [T1]. In particular, consider:

Theorem 0.2. Let $X$ be a compact, oriented, 4-manifold with $b_{2}^{+}>1$ and with a symplectic form $\omega$. Then

(1) The Poincaré dual of $c_{1}(K)$ is represented by the fundamental class of an embedded, symplectic curve.

(2) Let $e \in H_{2}(X ; \mathbb{Z})$ denote a homology class which is represented by an embedded sphere with self-intersection number -1 . Then $e$ is represented by a symplectically embedded 2-sphere and $\left\langle c_{1}(K), e\right\rangle= \pm 1$.

(3) If $c_{1}(K)$ has negative square, then $X$ can be blown down along a symplectic sphere of self-intersection -1 .

(4) Suppose that $X$ cannot be blown down along a symplectic sphere of selfintersection -1. Then the signature of the intersection form of $X$ is no smaller than $-\frac{4}{3}\left(1-b_{1}\right)-\frac{2}{3} b_{2}$. (The $b_{i}$ 's are the Betti-numbers of $X$.)

(5) If $c_{1}(K)$ has square zero and $X$ has no symplectically embedded 2-spheres with self-intersection -1, then $c_{1}(K)$ is Poincaré dual to a disjoint union of embedded, symplectic tori with zero self-intersection number. In fact, any class in $H^{2}(X ; \mathbb{Z})$ with non-zero Seiberg-Witten invariant is represented by disjoint, symplectically embedded tori with square zero.

(6) Symplectic manifolds have "simple type" in that only the dimension zero Seiberg-Witten invariants are non-zero. That is, $\mathrm{SW}(e)=0$ if $c_{1}(K) \cdot e-e \cdot e \neq$ 0 .

Note that Assertion (2) above is a refinement due to Daniel Ruberman of a somewhat weaker assertion in [T1]. Also, Assertion (6) can be proved using the afore-mentioned announced results of Donaldson.

Note that symplectic submanifolds are closely related to pseudo-holomorphic submanifolds. The definition of the latter requires the introduction of an almost complex structure $J$ for $X$. (This is an endomorphism whose square is minus the identity.) A submanifold $\Sigma$ is called pseudo-holomorphic (after Gromov [Gr]) when $J$ maps $T \Sigma$ to itself. An almost complex structure is said to be $\omega$-compatible when 
the bilinear form

$$
\omega(\cdot, J(\cdot))
$$

defines a Riemannian metric on $X$. If $\Sigma$ is pseudo-holomorphic for an $\omega$-compatible almost complex structure, then $\Sigma$ is also symplectic. Conversely, if $\Sigma$ is symplectic, then there is an $\omega$-compatible almost complex structure which makes $\Sigma$ pseudoholomorphic. Thus, the preceding theorems assert the existence of certain types of pseudo-holomorphic submanifolds.

Theorem 1.3 has applications to manifolds with $b_{2}^{+}=1$ also. As noted in [T1], the following result follows from Theorem 1.3 (with Proposition 7.1) and a theorem of Gromov in $[\mathrm{Gr}]$ :

Theorem 0.3. The manifold $\mathbb{C P}^{2}$ has a unique (up to symplectomorphism) symplectic structure.

The proofs of Theorems 0.1-0.3 were sketched in [T1], and further remarks are provided in Section 7 of this article. Section 1 of this article reviews the SeibergWitten equations and it provides, in its final subsection, an overview of the contents of the subsequent sections.

At this point, the author wishes to acknowledge a large debt of gratitude to Tomasz Mrowka for his advice, suggestions and support during the production of this manuscript.

\section{The Seiberg-Witten equations}

The Seiberg-Witten invariants of a compact, oriented, dimension 4 manifold $X$ constitute a map from the set of equivalence classes of $\operatorname{Spin}^{\mathbb{C}}$ structures on $X$ (covering the frame bundle) to the integers. They are defined when the characteristic number $b_{2}^{+}$in (0.1) is greater than 1 . (There is a more complicated structure in the case where $b_{2}^{+}=1$.) These invariants were first described in [W], and the reader is also referred to [KM1] and [T2]. (A more complete description is in preparation with multiple authors.) What follows is a brief description of the invariants. This section ends with the statement of Theorem 1.3 (which is the principal result in this article), a discussion of the strategy for the proof, and an outline of the contents of subsequent sections.

(a) Some spin geometry. Here is a five part digression to review some crucial 4-dimensional geometry.

Part 1. The Lie groups $\mathrm{SO}(4)$ and $\operatorname{Spin}^{\mathbb{C}}(4)$ can be given as

$$
\mathrm{SO}(4)=(\mathrm{SU}(2) \times \mathrm{SU}(2)) /\{ \pm 1\},
$$

and

$$
\operatorname{Spin}^{\mathbb{C}}(4)=(\mathrm{U}(1) \times \mathrm{SU}(2) \times \mathrm{SU}(2)) /\{ \pm 1\},
$$

where $\{ \pm 1\}$ acts on all factors in both cases in the obvious way.

Part 2. Fix a Riemannian metric on $X$. The metric defines the principal $\mathrm{SO}(4)$ bundle of orthonormal frames on $X$. A $\operatorname{Spin}^{\mathbb{C}}$ structure (denoted by $L$ ) is simply a lift of this $\mathrm{SO}(4)$ principal bundle to a $\operatorname{Spin}^{\mathbb{C}}(4)$ principal bundle. The set of equivalence classes of such lifts has, in a natural way, the structure of a principal $H^{2}(X ; \mathbb{Z})$ bundle over a point. This principal $H^{2}(X ; \mathbb{Z})$ bundle, Spin, is canonically 
defined and independent of the original choice of metric on $X$. One should think of the Seiberg-Witten invariant as a map from Spin to $\mathbb{Z}$.

Part 3. The group $\mathrm{SO}(4)$ has two evident representations into $\mathrm{SO}(3)=\mathrm{SU}(2) /\{ \pm 1\}$, which will be called $\lambda_{+}$and $\lambda_{-}$. They are distinguished by the convention that the associated $\mathbb{R}^{3}$ bundles to the frame bundle of $X$ be isomorphic to the bundles $\Lambda_{+}$ of self-dual 2-forms and $\Lambda_{-}$of anti-self-dual 2-forms, respectively.

Likewise, the group $\operatorname{Spin}^{\mathbb{C}}(4)$ has two evident representations, $s_{+}$and $s_{-}$, into $\mathrm{U}(2)=(\mathrm{U}(1) \times \mathrm{SU}(2)) /\{ \pm 1\}$. The convention is that the composition of $s_{+}$with the quotient homomorphism $\mathrm{U}(2) \rightarrow \mathrm{U}(2) /$ Center $=\mathrm{SO}(3)$ factors through $\mathrm{SO}(4)$ via $\lambda_{+}$. Anyway, with the preceding understood, given a $\operatorname{Spin}^{\mathbb{C}}$ structure $L$ on $X$, introduce the $\mathbb{C}^{2}$-vector bundles

$$
S_{+}, S_{-} \rightarrow X
$$

which are associated to $L$ via the representations $s_{+}$and $s_{-}$, respectively. These bundles inherit natural fiber metrics.

Let $L$ and $L \cdot e$ be elements in Spin, where $e \in H^{2}(X ; \mathbb{Z})$. Then, the bundles $S_{+}$ for these two $\operatorname{Spin}^{\mathbb{C}}$ structures are related by $S_{+}(L \cdot e)=S_{+}(L) \otimes E$, where $E$ is the complex line bundle with first Chern class $c_{1}(E)=e$.

Part 4. Clifford multiplication, $c$, maps $T^{*} X$ into the skew adjoint endomorphisms of $S_{+} \oplus S_{-}$; it is characterized by the equality $c(v)^{2}$ is multiplication by $-|v|^{2}$. In particular, $c$ induces maps

$$
\sigma: S_{+} \otimes T^{*} X \rightarrow S_{-}
$$

(by duality) and also $c_{+}: \Lambda_{+} \rightarrow \operatorname{End}\left(S_{+}\right)$. The adjoint of the latter will be denoted by

$$
\tau: \operatorname{End}\left(S_{+}\right) \rightarrow \Lambda_{+} \otimes \mathbb{C}
$$

it maps a self-adjoint endomorphism into an imaginary valued form. To be more explicit, let $\left\{e^{\nu}\right\}_{\nu=1}^{4}$ be an oriented, orthonormal frame at a point of $X$. Then $\tau\left(\eta \otimes \eta^{*}\right)=-2^{-1} \cdot\left\langle\eta, c\left(e^{\nu}\right) c\left(e^{\mu}\right) \eta\right\rangle\left(e^{\nu} \wedge e^{\mu}\right)$. Here, $\langle$,$\rangle denotes the Hermitian inner$ product on $S^{+}$.

Part 5. Let $A$ be a connection on $L \equiv \operatorname{det}\left(S_{+}\right)$. The connection $A$ with the LeviCivita connection on $T^{*} X$ induces a covariant derivative, $\nabla_{A}$ on $S_{+}$. This maps sections of $S_{+}$into sections of $S_{+} \otimes T^{*} X$. The composition of this last map with $\sigma$ in (1.4) defines the Dirac operator $D_{A}$, a first order elliptic operator mapping sections of $S_{+}$to sections of $S_{-}$. That is, if $\psi$ is a section of $S_{+}$, then the action of $D_{A}$ on $\psi$ is given by

$$
D_{A} \psi \equiv \sigma\left(\nabla_{A} \psi\right) .
$$

(b) The Seiberg-Witten equations. With the preceding understood, remark that the Seiberg-Witten equations are equations for a pair $(A, \psi)$, where $A$ is a connection on $L=\operatorname{det}\left(S_{+}\right)$and where $\psi$ is a section of $S_{+}$. These equations read

$$
D_{A} \psi=0 \quad \text { and } \quad P_{+} F_{A}=\frac{1}{4} \tau\left(\psi \otimes \psi^{*}\right),
$$

where $P_{+}: \Lambda^{2} T^{*} X \rightarrow \Lambda_{+}$is the orthogonal projection. It proves useful at times to consider perturbations of (1.7) which have the form

$$
D_{A} \psi=0 \quad \text { and } \quad P_{+} F_{A}=\frac{1}{4} \tau\left(\psi \otimes \psi^{*}\right)+\mu,
$$

where $\mu$ is a fixed, imaginary valued, anti-self-dual 2-form on $X$. 
The Seiberg-Witten invariant for the given $\operatorname{Spin}^{\mathbb{C}}$ structure $L \in$ Spin is obtained by making a suitable count of solutions of (1.6) or (1.7). Remark here that the group $C^{\infty}\left(X ; S^{1}\right)$ acts on the space of solutions to $(1.7)$; a map $\varphi$ sends $(A, \psi)$ to $\left(A-2 \varphi^{-1} d \varphi, \varphi \psi\right)$. Here, $S^{1}$ is thought of as the unit sphere in $\mathbb{C}$. (This group acts freely at solutions where $\psi$ is not identically zero.) The quotient of the space of solutions to $(1.7)$ by $C^{\infty}\left(S^{1} ; X\right)$ will be denoted by $M$. (The dependence on the Spin $^{\mathbb{C}}$ structure and on the choice of $\mu$ in (1.7) will usually be suppressed.)

Here are five crucial facts about $M$ :

Fact 1. When $b_{2}^{+} \geq 1$, the space of solutions to (1.7) and (1.8) will contain no points where $\psi \equiv 0$ for a generic metric or choice of $\mu$ as long as $c_{1}(L)$ is rationally non-zero. Here, generic means off a set of codimension $b_{2}^{+}$. (This follows from a theorem of Uhlenbeck in [FU].)

Fact 2 . The space $M$ has naturally the structure of a real analytic variety. When $b_{2}^{+} \geq 1$, the space $M$ will be a smooth manifold for a generic choice of $\mu$ in (1.8). (Here, generic means a Baire subset of $C^{\infty}\left(\Lambda_{+}\right)$.) The dimension of this manifold is computed with the help of the Atiyah-Singer index theorem to be

$$
d=-\frac{1}{4}(2 \chi(X)+3 \operatorname{sign}(X))+\frac{1}{4} c_{1}(L) \cdot c_{1}(L) .
$$

Here, $\chi$ is the Euler characteristic of $X$ and $\operatorname{sign}(X)$ is the signature. Also, the notation $u \cdot v$ for classes $u, v \in H^{2}(X ; \mathbb{Z})$ denotes the evaluation of their cup product on the fundamental class of $X$.

Fact 3. A choice of orientation for the line

$$
\operatorname{det}\left(H^{0}(X ; \mathbb{R})\right) \otimes \operatorname{det}\left(H^{1}(X ; \mathbb{R})\right) \otimes \operatorname{det}\left(H^{2+}(X ; \mathbb{R})\right)
$$

serves to orient $M$. (The orientation of a point is a choice of \pm 1 assigned to said point.)

Fact 4. Fix a base point in $X$ and let $C_{0}^{\infty}\left(S^{1} ; X\right)$ denote the subset of maps which map the base point to 1 . Let $M^{0}$ denote the quotient of the space of solutions to (1.8) by the latter group. Where $M$ is a smooth manifold, the projection $M^{0} \rightarrow M$ defines a principal $S^{1}$ bundle.

Fact 5. The space $M$ is compact.

With the preceding understood, here is the definition of the Seiberg-Witten invariant.

Definition 1.1. Let $X$ be a compact, oriented 4-dimensional manifold with $b_{2}^{+}>1$ and let $L \in \operatorname{Spin}$ be a Spin ${ }^{\mathbb{C}}$ structure on $X$. Choose an orientation for (1.9). The Seiberg-Witten invariant $\mathrm{SW}(L)$ for $L$ is defined as follows:

(a) When $d<0$ in (1.9), the invariant is defined to be zero.

(b) When $d=0$ in (1.9), choose $\mu$ in (1.8) to make $M$ a smooth manifold. Then this $M$ is a finite union of signed points and the Seiberg-Witten invariant is the sum over these points of the corresponding \pm 1 's.

(c) When $d>0$ in (1.9), choose $\mu$ in (1.8) to make $M$ a smooth manifold. This $M$ is compact and oriented so has a fundamental class. The Seiberg-Witten invariant is obtained by pairing this fundamental class with the maximum cup product of the first Chern class of the line bundle $M^{0} \times{ }_{S^{1}} \mathbb{C}$. 
Note that the dimensions of $M$ and $b_{2}^{+}+b_{1}$ have opposite parity; thus, the Seiberg-Witten invariants are zero when $b_{2}^{+}+b_{1}$ is even.

The facts listed above then yield

Proposition 1.2. Let $X$ be a compact, oriented, connected, dimension 4 manifold with $b_{2}^{+}>1$. Then $\mathrm{SW}$ defines a map from Spin to $\mathbb{Z}$ which depends only on the underlying smooth structure of $X$. That is, the value of $\mathrm{SW}(L)$ is independent of the choice of metric and perturbing form $\mu$ in (1.8). It depends only on $L$ up to isomorphism. Furthermore, the assignment of $\mathrm{SW}$ to a $\mathrm{Spin}^{\mathbb{C}}$ structure is invariant under self-diffeomorphisms of $X$ in the following sense: if $\varphi$ is a diffeomorphism of $X$, then the value of $\mathrm{SW}$ on $\varphi^{*} L$ is, up to sign, the same as the value of $\mathrm{SW}$ on $L$.

(c) Symplectic manifolds. A 2-form $\omega$ on an oriented 4-manifold $X$ is symplectic when

$$
d \omega=0 \quad \text { and } \quad \omega \wedge \omega \neq 0
$$

everywhere. Furthermore, the 4 -form $\omega \wedge \omega$ will be required to orient $X$. A 4manifold $X$ with a symplectic form $\omega$ will be called a symplectic 4-manifold.

Every symplectic manifold has a canonical complex line bundle $K$ called the canonical bundle. Fix a Riemannian metric on $X$; then $K$ can be identified as the orthogonal 2-plane bundle to the projection of $\omega$ into $\Lambda_{+}$. (The fact that $\omega \wedge \omega \neq 0$ implies that this last projection is nowhere zero.) Alternately, $K$ can be defined by choosing an almost complex structure $T X$ which is compatible (in the sense of Gromov [Gr]) for $\omega$. In this case, $K$ is $\operatorname{det}\left(T^{1,0} X\right)$. The specification of such an almost complex structure is equivalent to the specification of a metric on $X$ for which $\omega$ is self-dual. Note that when $t \rightarrow \omega_{t}$ is a continuous, 1-parameter family of symplectic forms on $X$, then the canonical bundles for $\left(X, \omega_{0}\right)$ and $\left(X, \omega_{1}\right)$ will be isomorphic.

A symplectic manifold also has a canonical $\operatorname{Spin}^{\mathbb{C}}$ structure (see [T2]). Indeed, use a metric for which $\omega$ is self-dual with length $\sqrt{2}$. For such a metric, the canonical Spin $^{\mathbb{C}}$ structure is characterized by the fact that its associate bundle $S_{+}$is naturally isomorphic to $\mathbb{I} \oplus K^{-1}$, where $\mathbb{I}$ is the trivial complex line bundle. Here, $\omega$ acts by Clifford multiplication on the $\mathbb{I}$ summand with eigenvalue $-2 i$, and it acts on $K^{-1}$ summand with eigenvalue $+2 i$. (When $t \rightarrow \omega_{t}$ is a continuous, 1-parameter family of symplectic forms on $X$, then the canonical $\operatorname{Spin}^{\mathbb{C}}$ structures for $\omega_{0}$ and $\omega_{1}$ can be naturally identified.)

With the preceding understood, remark that the line bundle $K^{-1}$ has a canonical (up to gauge equivalence) connection $A_{0}$ which is characterized as follows: If $A$ is a covariant derivative on $K^{-1}$, then the spin covariant derivative $\nabla_{A}$ induces a covariant derivative on the $\mathbb{I}$ summand of $S_{+}$which is given by

$$
\nabla_{A} \equiv \frac{1}{2}\left(1+\frac{i}{2} c_{+}(\omega)\right) \nabla_{A}: C^{\infty}(\mathbb{I}) \rightarrow C^{\infty}\left(\mathbb{I} \otimes T^{*} X\right) .
$$

With (1.12) understood, note that $A_{0}$ is characterized by the requirement that $\nabla_{A_{0}}$ annihilate a non-trivial section, $u_{0}$. This $u_{0}$ will be taken to have norm one. Note that

$$
D_{A_{0}} u_{0}=0,
$$

which is a consequence of the fact that $d \omega=0$. (This is proved in [T2].) 
The defining of this canonical $\operatorname{Spin}^{\mathbb{C}}$ structure allows one to identify Spin, the set of equivalence classes of $\operatorname{Spin}^{\mathbb{C}}$ structures on $X$, with the set of equivalence classes of complex line bundles over $X$. (The latter is, of course, the same as $H^{2}(X ; \mathbb{Z})$.) The Spin ${ }^{\mathbb{C}}$ structure which corresponds to a given complex line bundle $E$ is characterized by the fact that its bundle $S_{+}$is given by

$$
S_{+}=E \oplus\left(K^{-1} \otimes E\right) .
$$

Note that Clifford multiplication by $\omega$ on $S_{+}$in (1.14) preserves the splitting with the summand $E$ having eigenvalue $-2 i$.

Note that the line bundle $L=\operatorname{det}\left(S_{+}\right)$for (1.14) is $K^{-1} \otimes E^{2}$. Thus, a connection $A$ on $L$ is determined by the canonical connection $A_{0}$ on $K^{-1}$ and by the choice of connection, $a$, on $E$. The relationship between $A$ and $a$ is characterized as follows: Let $\alpha$ be a section of $E$. To avoid confusion, $\alpha \cdot u_{0}$ will denote the corresponding section of the $E$ summand in $S_{+}$as given in (1.14). And, with this understood, the spin covariant derivative (using $\nabla_{A}$ ) of $\alpha \cdot u_{0}$ is related to the $\nabla_{a}$ covariant derivative of $\alpha$ by the formula

$$
\nabla_{A}\left(\alpha \cdot u_{0}\right)=\left(\nabla_{a} \alpha\right) \cdot u_{0}+\alpha \cdot \nabla_{A_{0}} u_{0} .
$$

(Note that $\nabla_{A_{0}} u_{0}$ is a section of the $K^{-1} \otimes T^{*} X$ summand of $S_{+} \otimes T^{*} X$.)

When $\beta$ is a section of the $K^{-1} \otimes E$ summand in (1.14), introduce the notation

$$
\nabla_{A}^{\prime} \beta \equiv \frac{1}{2}\left(1-\frac{i}{2} c_{+}(\omega)\right) \nabla_{A} \beta,
$$

which is a section of the $\left(K^{-1} \otimes E\right) \otimes T^{*} X$ summand of $S_{+} \otimes T^{*} X$.

(d) The perturbation. There is a natural 1-parameter family of choices for the 2 -form $\mu$ in (1.8) on a symplectic manifold. The family is parameterized by a real number $r \geq 0$ and is given by

$$
\mu=-\frac{i \cdot r}{4} \omega+P_{+} F_{A_{0}} .
$$

Here, $P_{+}: \Lambda^{2} T^{*} X \rightarrow \Lambda_{+}$is the metric's orthogonal projection onto the self-dual forms. With this perturbation choice, the Seiberg-Witten equations read

$$
D_{A} \psi=0 \quad \text { and } \quad P_{+} F_{A}=P_{+} F_{A_{0}}+\frac{1}{4}\left(\tau\left(\psi \otimes \psi^{*}\right)-i \cdot r \cdot \omega\right) .
$$

When analyzing (1.18), it proves useful to write $S_{+}$as in (1.14) and to write the section $\psi$ as

$$
\psi=r^{1 / 2} \cdot\left(\alpha u_{0}+\beta\right),
$$

where $\alpha$ is a section of $E$ and where $\beta$ is a section of the $K^{-1} \otimes E$ summand in (1.14). Then, with $\psi$ given as in (1.19), the Seiberg-Witten equations in (1.18) are equivalent to

$$
\begin{gathered}
\sigma\left(u_{0} \otimes \nabla_{a} \alpha\right)+D_{A} \beta=0, \\
P_{+} F_{a}=-\frac{i}{8} r \cdot\left(1-|\alpha|^{2}+|\beta|^{2}\right) \cdot \omega+\frac{i \cdot r}{4}\left(\alpha \beta^{*}+\alpha^{*} \beta\right) .
\end{gathered}
$$

Here, $\alpha \beta^{*}$ and $\alpha^{*} \beta$, being respective sections of $K$ and $K^{-1}$, are identified as summands of $\Lambda_{+} \otimes \mathbb{C}$. 
(e) The main theorem. With the preceding equations understood, here is the principal result in this paper:

Theorem 1.3. Let $X$ be a compact 4-manifold with symplectic form $\omega$. Fix a Riemannian metric which makes the symplectic form anti-self-dual and of length $\sqrt{2}$. Fix a complex line bundle $E \rightarrow X$. Let $\left\{\Omega_{i} \subset X\right\}$ be a finite (maybe empty) set of closed sets. Assume that there exists an unbounded sequence of values for the parameter $r$ in (1.20) for which (1.20) has a solution for the $\mathrm{Spin}^{\mathbb{C}}$ structure in (1.14). And, suppose that for each of these $r$ values, there is such a solution with $\Omega_{i} \cap \alpha^{-1}(0) \neq \varnothing$ for all $i$. Then there exists a smooth, compact, complex (not necessarily connected) curve $\Sigma$ and a pseudo-holomorphic map $\varphi: \Sigma \rightarrow X$ with $\varphi_{*}[\Sigma]$ equal to the Poincaré dual to $c_{1}(E)$ and with $\Omega_{i} \cap$ image $(\varphi) \neq \varnothing$ for all $i$. (The almost complex structure on $T X$ is defined by the metric and the symplectic form.)

(Remark that a map from a complex curve into $X$ is called pseudo-holomorphic when its differential everywhere intertwines the action of the complex structure on the curve with the action of $J$. Thus, the image of a pseudo-holomorphic embedding is a pseudo-holomorphic submanifold.)

Let $\left\{r_{n}\right\}$ be an unbounded sequence of $r$ values as in Theorem 1.3, and let $\left\{\left(A_{n}, \alpha_{n}, \beta_{n}\right)\right\}$ be the corresponding sequence of solutions. The proof of Theorem 1.3 will show that the sequence of subsets $\left\{\alpha_{n}^{-1}(0)\right\} \subset X$ converges to $\varphi(\Sigma)$ in the following sense: There is a subsequence with the property that

$$
\lim _{n \rightarrow \infty} \sup _{x \in \varphi(\Sigma)} \operatorname{dist}\left(x, \alpha_{n}^{-1}(0)\right)+\sup _{x \in \alpha_{n}^{-1}(0)} \operatorname{dist}(x, \varphi(\Sigma))=0 .
$$

Here is the strategy for the proof of Theorem 1.3: Take a sequence $\left\{r_{n}\right\}$ of $r$ values for Theorem 1.3 with the corresponding sequence $\left(A_{n},\left(\alpha_{n}, \beta_{n}\right)\right)$ of solutions to (1.20). Now consider the sequence $\left\{\frac{i}{2 \pi} F_{A_{n}}\right\}$ as a sequence of currents $\mathcal{F}_{n}$, where

$$
\mathcal{F}_{n}(\mu) \equiv \int \frac{i}{2 \pi} F_{A_{n}} \wedge \mu \text {. }
$$

(A current is an element in the dual space to the Frechet space of smooth sections of $\Lambda^{*} T^{*} X$.) Various properties of this sequence will be established with the ultimate goal being an assertion to the effect that the sequence $\left\{\mathcal{F}_{n}\right\}$ has a subsequence which converges to a current, $\mathcal{F}$, whose support is a closed set $C$ with finite 2dimensional Hausdorff measure. (A current has support on a set $C$ if it annihilates all forms with compact support in the complement of $C$.) The current $\mathcal{F}$ will be used to define an integer valued, homotopy invariant (called $I$ ) of a certain class of maps from 2-dimensional disks into $X$. The allowed maps are those for which $C$ intersects the closure of the image of the disk only in the image of the disk. (This $I$ is defined by taking the limit of the integrals of $\left\{\frac{i}{2 \pi} \mathcal{F}_{A_{n}}\right\}$ over the disk in question.) A crucial point is that this homotopy invariant assigns a positive integer to any symplectically embedded disk with non-trivial intersection, but compactly supported (in the preceding sense) intersection with $C$. These properties of the current $\mathcal{F}$ are established in Section 5 .

Section 6 continues the proof of Theorem 1.3 by exploiting the positivity of $I$ on symplectic disks to prove that $C$ must be the image of a complex curve by a pseudo-holomorphic map. It is pertinent to consider here the following classical 
analog:

Let $C \subset \mathbb{C}^{2}$ be a codimension 2 submanifold with positive local intersection with all complex lines. Then $C$ is complex analytic.

(See the introduction to Section 6 for a proof.) The results in Section 6 make no specific references to the Seiberg-Witten equations per se. The Seiberg-Witten equations enter only Section 5 to give the current $\mathcal{F}$.

The results in Section 6 are reminiscent of the discussion in Section 5 of [Ki].

As for Sections 2-4, they provide the essential a priori estimates on solutions to (1.20) for use in Section 5.

(f) An outline of the remaining sections. As remarked, Sections 2-4 provide the key analytic estimates for the proof of Theorem 1.3. Here are their key estimates with the relevant reference to the text:

$$
\begin{aligned}
& \text { (1) }|\alpha| \leq 1+\frac{z}{r} \quad \text { (Proposition 2.1). } \\
& \text { (2) }|\beta|^{2} \leq \frac{z}{r}\left(1-|\alpha|^{2}\right)+\frac{z^{\prime}}{r^{3}} \quad \text { (Proposition 2.3). } \\
& \text { (3) } \left.\left|P_{ \pm} F_{a}\right| \leq \frac{r}{4 \sqrt{2}}\left(1-|\alpha|^{2}\right)+z \quad \text { (Propositions } 2.4 \text { and } 3.4\right) . \\
& \text { (4) } \left.\left|\nabla_{a} \alpha\right|^{2} \leq z \cdot r \cdot\left(1-|\alpha|^{2}\right)+z^{\prime} \quad \text { (Proposition } 2.8\right) . \\
& \text { (5) }\left|\nabla_{A}^{\prime} \beta\right|^{2} \leq z \cdot\left(1-|\alpha|^{2}\right)+\frac{z^{\prime}}{r} \quad(\text { Proposition } 2.8) . \\
& \text { (6) If } q \text { is one of }\left\{r^{3 / 2} \beta, r \cdot\left(1-|\alpha|^{2}\right), F_{a}, r^{1 / 2} \nabla_{a} \alpha, r \cdot \nabla_{A}^{\prime} \beta\right\}, \text { then } \\
& |q(x)| \leq z \cdot r \cdot \exp \left(\frac{1}{z} r^{1 / 2} \operatorname{dist}\left(x, \alpha^{-1}(0)\right)\right) \quad(\text { Proposition 4.4). }
\end{aligned}
$$

Here, $z$ and $z^{\prime}$ are constants which are determined solely by $c_{1}(E)$ and the Riemannian metric.

Also key to the proof is Proposition 3.1 which provides a "monotonicity formula" for the function $e$ on $(0,1) \times X$ whose value at a pair $(s, x)$ is defined to be the integral over the ball of radius $s$ with center $x$ of $\left.\frac{r}{4}|1-| \alpha\right|^{2} \mid$. Proposition 3.1 asserts (in part) that

$$
\begin{aligned}
& \text { (1) } e(s, x) \leq z \cdot s^{2}, \\
& \text { (2) } e(s, x) \geq \frac{1}{z} s^{2} \quad \text { if } s \geq r^{-1 / 2} \text { and } \alpha(x)=0 .
\end{aligned}
$$

The estimates in (1.24) and (1.25) are used in Section 4 to provide a description of $\alpha^{-1}(0)$ at length scales which are $\mathcal{O}\left(r^{-1 / 2}\right)$. The key observation here is that for larger $r, \alpha^{-1}(0)$ is very close to being pseudo-holomorphic at these length scales. Proposition 4.2 makes this statement precise. Note that Proposition 4.1 describes the solutions to the Seiberg-Witten equations on $\mathbb{C}^{2}$. (The story here is not as simple as one might initially imagine.)

As remarked above, Section 5 uses the preceding estimates to prove various statements about a limit $\mathcal{F}$ of the distributions in (1.22). In particular, (1.25) is used to prove that the support of $\mathcal{F}$ has finite 2-dimensional Hausdorff measure. And, (1.24) is used to prove that $\mathcal{F}$ is a distribution of type 1-1 which has positive, integer pairing with any pseudo-holomorphic disk which intersects $\mathcal{F}$ 's support in its interior. 
As remarked also above, Section 6 uses the facts about $\mathcal{F}$ from Section 5 to prove that the support of $\mathcal{F}$ is the image of a complex curve by a pseudo-holomorphic map. Section 6 completes the proof of Theorem 1.3.

Section 7 starts with Proposition 7.1. This proposition asserts a genericity result which implies that the pseudo-holomorphic maps in Theorem 1.3 are embeddings if the metric is suitably generic. After proving Proposition 7.1, Section 7 goes on to discuss the applications to Theorems $0.1-0.3$ of the introduction.

\section{Estimates}

The purpose of this section is to derive some very useful estimates for solutions to (1.20) when the $\operatorname{Spin}^{\mathbb{C}}$ structure has its $S_{+}$bundle as in (1.14). For the most part, these estimates will come through applications of the maximum principle. (The reader can find baby versions of most of the estimates below in [T3].)

(a) Apriori bounds on $(\alpha, \beta)$. The starting point for the bounds on $\alpha$ and $\beta$ is the equation $D_{A} \psi=0$. Here, $\psi=r^{1 / 2}\left(\alpha u_{0}+\beta\right)$. This last equation implies that $D_{A}^{2} \psi=0$ too. It is the latter equation which is useful. After commuting derivatives, it reads

$$
\nabla_{A}^{*} \nabla_{A} \psi+\frac{R}{4} \psi+\frac{1}{2} \cdot c_{+}\left(P_{+} F_{A}\right) \psi=0
$$

(Here, $\nabla_{A}^{*}$ is the formal $L^{2}$ adjoint of $\nabla_{A}$.)

The first application of (2.1) bounds $\left(|\alpha|^{2}+|\beta|^{2}\right)$ with the analog of an argument from [KM1].

Proposition 2.1. There is a constant $z$ which depends only on the Riemannian metric and which has the following significance: Suppose that the $\operatorname{Spin}^{\mathbb{C}}$ structure is given by (1.14), and suppose that $(A,(\alpha, \beta))$ solve (1.20) for some $r \geq 0$. Then

$$
|\alpha|^{2}+|\beta|^{2} \leq\left(1+z \cdot r^{-1}\right) \text {. }
$$

Proof of Proposition 2.1. To begin, take the inner product of (2.1) with $\psi$. After employing (1.20), the result reads

$$
\frac{1}{2} d^{*} d|\psi|^{2}+\left|\nabla_{A} \psi\right|^{2}+\frac{1}{4}|\psi|^{2}\left(|\psi|^{2}-r\right)-z_{1} \cdot|\psi|^{2} \leq 0 .
$$

(Here, $d^{*}$ is the formal $L^{2}$ adjoint of the exterior derivative $d$.) In (2.2), $z_{1}$ is a constant which depends on the Riemannian metric. It follows from (2.2) and the maximum principle that $|\psi|^{2}$ can have no local maxima where $|\psi|^{2}>r+4 z_{1}$. Since $X$ is assumed compact, the lemma follows.

(b) Separate equations for $\alpha$ and for $\beta$. To progress further, it is necessary to project (2.1) onto the $E$ summand of $S_{+}$and also onto the $K^{-1} \otimes E$ summand of $S_{+}$. To consider the projection of (2.1) onto the $E$ summand, take the inner product (in $S_{+}$) of both sides of (2.1) with $\alpha u_{0}$. After an appeal to (1.20) and some simple manipulations, one finds the following equation for $|\alpha|^{2}$ :

$$
\begin{aligned}
\frac{1}{2} d^{*} d|\alpha|^{2}+\left|\nabla_{a} \alpha\right|^{2}+\frac{r}{4}|\alpha|^{2}\left(|\alpha|^{2}-1\right. & \left.+|\beta|^{2}\right) \\
& +\operatorname{Re}\left\langle\alpha u_{0}, b \cdot \nabla_{A} \beta\right\rangle+\operatorname{Re}\left\langle\alpha u_{0}, q \beta\right\rangle=0 .
\end{aligned}
$$

Here, $b$ and $q$ are defined from $\nabla \omega$; in particular, neither depends on either $(A,(\alpha, \beta))$ nor $r$. (Both $b$ and $q$ vanish when $X$ is a complex surface and $\omega$ is the Kähler form; 
up to some multiple, $b$ defines the torsion tensor for the almost complex structure and $q$ is obtained from derivatives of $b$.)

To consider the projection of (2.1) onto the $K^{-1} \otimes E$ summand of $S_{+}$, take the inner product (in $S_{+}$) of (2.1) with $\beta$. After an appeal to (1.20) and some simple manipulations, a differential inequality for $|\beta|^{2}$ appears:

$$
\frac{1}{2} d^{*} d|\beta|^{2}+\left|\nabla_{A} \beta\right|^{2}+\frac{r}{4}|\beta|^{2}\left(|\beta|^{2}+1+|\alpha|^{2}\right)-z_{1}|\beta|^{2} \leq z_{2}|\beta|\left|\nabla_{a} \alpha\right| .
$$

Here, $z_{1}$ and $z_{2}$ are constants which depend solely on the form $\omega$ and the Riemannian metric. (In particular, they are independent of the solution $(A,(\alpha, \beta))$ and of the parameter $r$.)

The lemma below turns out to be key to essentially all of the maximum principle applications in the remainder of this section:

Lemma 2.2. There are constants $\kappa_{1}, \kappa_{2}, \kappa_{3} \geq 1$ which depend only on the Riemannian metric and which have the following significance: Let the $\operatorname{Spin}^{\mathbb{C}}$ structure be given by (1.14), and let $(A,(\alpha, \beta))$ solve (1.20) for some $r \geq \kappa_{1}$. Let $\zeta \in\left(0, \frac{r}{2 \kappa_{1} \kappa_{2}}\right)$ and $\delta \geq \kappa_{3}$. Set

$$
u \equiv\left(1-|\alpha|^{2}\right)-\zeta|\beta|^{2}+\frac{\delta}{\zeta r} .
$$

Then

$$
\frac{1}{2} d^{*} d u+\frac{r}{4}|\alpha|^{2} u \geq\left(1-\frac{\zeta \cdot \kappa_{1}}{r}\right)\left|\nabla_{a} \alpha\right|^{2}+\frac{\zeta r}{8}|\beta|^{2}+\frac{\zeta}{2}\left|\nabla_{A}^{\prime} \beta\right|^{2}+\frac{\delta}{8 \zeta}|\alpha|^{2} .
$$

Proof of Lemma 2.2. It follows from (2.4) (with the help of the triangle inequality) that the function $|\beta|^{2}$ obeys the following differential inequality when $r>16 z_{1}$ with $z_{1}$ as in (2.4):

$$
\frac{1}{2} d^{*} d|\beta|^{2}+\frac{r}{4}|\alpha|^{2}|\beta|^{2} \leq-\frac{r}{8}|\beta|^{2}-\left|\nabla_{A} \beta\right|^{2}+\frac{z_{3}}{r}\left|\nabla_{a} \alpha\right|^{2},
$$

where $z_{3}$ is a constant which is independent of $(A,(\alpha, \beta))$ and $r$.

Now, with $\rho>0$ a given constant, introduce the function

$$
w \equiv 1-|\alpha|^{2}+\frac{\rho}{r} .
$$

Equation (2.3) implies the following equality for $w$ :

$$
\begin{aligned}
& \frac{1}{2} d^{*} d w+\frac{r}{4}|\alpha|^{2} w \\
& \quad=\left|\nabla_{a} \alpha\right|^{2}+\frac{r}{4}|\alpha|^{2}|\beta|^{2}+\frac{\rho}{4}|\alpha|^{2}+\operatorname{Re}\left\langle\alpha u_{0}, b \cdot \nabla_{A}^{\prime} \beta\right\rangle+\operatorname{Re}\left\langle\alpha u_{0}, q \beta\right\rangle .
\end{aligned}
$$

And, this last equation implies that

$$
\frac{1}{2} d^{*} d w+\frac{r}{4}|\alpha|^{2} w \geq\left|\nabla_{a} \alpha\right|^{2}+\frac{r}{4}|\alpha|^{2}|\beta|^{2}-\frac{z_{4}}{\rho}\left(\left|\nabla_{A}^{\prime} \beta\right|^{2}+|\beta|^{2}\right) .
$$

Here, $z_{4}$ depends only on the sup norm of $b$ and $q$.

Lemma 2.2 follows directly from (2.7) and (2.10). 
(c) Pointwise estimates on $\beta$. The first application of Lemma 2.2 is the following pointwise estimate for $\beta$ :

Proposition 2.3. There are constants $z$ and $z^{\prime}$ which depend only on the Riemannian metric and on $\omega$ and which have the following significance: Let the $\operatorname{Spin}^{\mathbb{C}}$ structure be given by (1.14), and suppose that $(A,(\alpha, \beta))$ solve (1.20) for some $r \geq 0$. Then

$$
|\beta|^{2} \leq \frac{z}{r}\left(1-|\alpha|^{2}+\frac{z^{\prime}}{r^{2}}\right)
$$

Proof of Proposition 2.3. Take $\zeta \equiv \frac{r}{2 \kappa_{1} \kappa_{2}}$ and take $\delta \equiv \kappa_{3}$ in Lemma 2.2. With these choices, $\frac{1}{2} d^{*} d u+\frac{r}{4}|\alpha|^{2} u \geq 0$, and the maximum principle implies that $u$ cannot have a negative minimum. Thus, $u \geq 0$ and the lemma follows.

(d) Pointwise estimates on curvature. The curvature $F_{a}$ of the connection $a$ on $E$ can be bounded using (1.20). The bound for $P_{+} F_{a}$ follows by inspection:

$$
\left|P_{+} F_{a}\right|=\frac{r}{4 \sqrt{2}}\left(\left(1-|\alpha|^{2}\right)^{2}+2|\beta|^{2}\left(1+|\alpha|^{2}\right)+|\beta|^{4}\right)^{1 / 2} .
$$

The bound on $P_{-} F_{a}$ is harder to come by; but here it is:

Proposition 2.4. Fix a complex line bundle $E \rightarrow X$. There are constants $z$ and $z^{\prime}$ which are determined by $c_{1}(E)$ and the Riemannian metric and which have the following significance: Let $E$ be a complex line bundle on $X$. Let $r \geq 1$, and suppose that $(A,(\alpha, \beta))$ is a solution to (1.20) for the $\operatorname{Spin}^{\mathbb{C}}$ structure with $S_{+}$as in (1.14). Then

$$
\left|P_{ \pm} F_{a}\right| \leq \frac{r}{4 \sqrt{2}}\left(1+\frac{z}{r^{1 / 2}}\right) \cdot\left(1-|\alpha|^{2}\right)+z^{\prime} .
$$

The estimate for $P_{+} F_{A}$ follows from Proposition 2.3 and (2.12). The proof of this proposition for $P_{-} F_{A}$ is a five step affair which occupies the remainder of this subsection.

Step 1. The first step in the proof derives a 2nd order differential inequality for $\left|P_{-} F_{a}\right|$ which allows for applications of the maximum principle. To begin, note first that $d P_{-} F_{a}=-d P_{+} F_{a}$ because of the Bianchi identity. Therefore,

$$
P_{-} d^{*} d\left(P_{-} F_{a}\right)=P_{-} d^{*} d\left(P_{+} F_{a}\right) .
$$

Use (1.20) for the right side of (2.14) and use a standard Bochner-Weitzenböck formula for the left side in order to rewrite (2.14) as the following equation for $\mu \equiv-i \cdot P_{-} F_{a}$ :

$$
\frac{1}{2} \nabla^{*} \nabla \mu+\mathcal{R} \mu=-P_{-} d^{*} d\left\{-\frac{r}{8}\left(1-|\alpha|^{2}+|\beta|^{2}\right) \cdot \omega+\frac{r}{4}\left(\alpha \beta^{*}+\alpha^{*} \beta\right)\right\} .
$$

Here, $\mathcal{R}$ is a certain endomorphism of $\Lambda_{-}$which is constructed from the scalar curvature and anti-self-dual Weyl curvature of the Riemannian metric. (See, e.g., Appendix C in [FU].)

To put (2.15) into a useful form, it is necessary to rewrite the right side. To begin, use the first equation in (1.20) to derive

$$
\begin{aligned}
d^{*} d & \left(\frac{r}{8}\left(1-|\alpha|^{2}+|\beta|^{2}\right) \cdot \omega-\frac{r}{4}\left(\alpha \beta^{*}+\alpha^{*} \beta\right)\right) \\
& =-\frac{i r}{8}\left(\alpha \nabla_{a} \alpha^{*}-\alpha^{*} \nabla_{a} \alpha+\beta \nabla_{A}^{\prime} \beta^{*}-\beta^{*} \nabla_{A}^{\prime} \beta-\alpha \beta^{*} k+\alpha^{*} \beta k^{*}\right),
\end{aligned}
$$


where $k$ is a section of $K^{-1} \otimes T^{*} X$ which is defined from the covariant derivatives of $\omega$. (To be precise, identify $K^{-1}$ in the standard manner as a summand in the canonical spin bundle's $S_{+}$(take $E$ trivial in (1.14))). With this understood, then $k \equiv \frac{i}{2} \nabla \omega \cdot u_{0}$.) Then, taking the exterior derivative of both sides of (2.16) gives

$$
\begin{aligned}
d^{*} d\left(\frac{r}{8}\left(1-|\alpha|^{2}+|\beta|^{2}\right) \cdot \omega-\right. & \left.\frac{r}{4}\left(\alpha \beta^{*}+\alpha^{*} \beta\right)\right) \\
=+\frac{r}{4} i F_{a}\left(|\alpha|^{2}+|\beta|^{2}\right)+\frac{i r}{4}\left(\nabla_{a} \alpha^{*}\right. & \left.\wedge \nabla_{a} \alpha+\nabla_{A}^{\prime} \beta^{*} \wedge \nabla_{A}^{\prime} \beta\right) \\
& \quad-\frac{i r}{8} d\left(\alpha \beta^{*} k-\alpha^{*} \beta k^{*}\right)+\frac{r}{4} i \kappa \cdot|\beta|^{2} .
\end{aligned}
$$

Here, $\kappa+F_{a}$ is the curvature of the covariant derivative $\nabla_{A}^{\prime}$ on the line bundle $K^{-1} \otimes E$. Thus, $\kappa$ is independent of $(A,(\alpha, \beta))$ and $r$.

Now substitute the right side of (2.17) into the right side of (2.15) to derive the following equation for $\mu \equiv-i P_{-} F_{a}$ :

$$
\begin{aligned}
\frac{1}{2} \nabla^{*} \nabla \mu+\frac{r}{4}\left(|\alpha|^{2}+|\beta|^{2}\right) \mu+\mathcal{R} \mu= & \frac{i r}{4} P_{-}\left(\nabla_{a} \alpha^{*} \wedge \nabla_{a} \alpha+\nabla_{A}^{\prime} \beta^{*} \wedge \nabla_{A}^{\prime} \beta\right) \\
& -\frac{i r}{8} P_{-} d\left(\alpha \beta^{*} k-\alpha^{*} \beta k^{*}\right)+\frac{r}{4} i \kappa \cdot|\beta|^{2} .
\end{aligned}
$$

Let $s \equiv\left|P_{-} F_{a}\right|$ (which is $|\mu|$ ). This function $s$ obeys the following differential inequality:

$$
\begin{aligned}
\frac{1}{2} d^{*} d s+\frac{r}{4}\left(|\alpha|^{2}+|\beta|^{2}\right) s \leq & |\mathcal{R}| s+\frac{r}{4 \sqrt{2}}\left(\left|\nabla_{a} \alpha\right|^{2}+\left|\nabla_{A}^{\prime} \beta\right|^{2}\right) \\
& +z \cdot r\left(|\alpha||\beta|+|\alpha|\left|\nabla_{A}^{\prime} \beta\right|+|\beta| \cdot\left|\nabla_{a} \alpha\right|+|\beta|^{2}\right)
\end{aligned}
$$

Here, $z$ is a constant which is independent of $(A,(\alpha, \beta))$ and $r$.

Step 2. This step adds and subtracts various terms (of known size) from $s$ in order to obtain a differential inequality as in (2.19) with fewer terms on the right side. The result is summarized by

Lemma 2.5. There are positive constants $\kappa_{1}, \kappa_{2}, \kappa_{3}$ which depend only on the Riemannian metric and on $\omega$ and which have the following significance: Let the $\mathrm{Spin}^{\mathbb{C}}$ structure be given by (1.14), and suppose that $(A,(\alpha, \beta))$ solve (1.20) for some $r \geq \kappa_{1}$. Then, the functions

$$
q_{0} \equiv \frac{r}{4 \sqrt{2}}\left(1+\frac{1}{r} \kappa_{1}\right) \cdot\left(1-|\alpha|^{2}\right)-\kappa_{2} \cdot r \cdot|\beta|^{2}+\kappa_{3}
$$

and $s=\left|P_{-} F_{a}\right|$ obey

$$
\frac{1}{2} d^{*} d\left(s-q_{0}\right)+\frac{r}{4}|\alpha|^{2}\left(s-q_{0}\right) \leq|\mathcal{R}| s .
$$

Proof of Lemma 2.5. This lemma is a direct consequence of Lemma 2.2 and (2.19).

Step 3. This step bounds $s-q_{0}$ from above in terms of a certain non-negative function $q$. The function $q$ satisfies the differential equation

$$
\frac{1}{2} d^{*} d q+\frac{r}{4}|\alpha|^{2} q=|\mathcal{R}| s .
$$

Standard existence theorems find $q$ as long as $\alpha$ doesn't vanish identically. The vanishing of $\alpha$ is precluded by 
Lemma 2.6. There is a constant $z$ which is determined by the Riemannian metric and which has the following significance: Fix a complex line bundle $E \rightarrow X$. For $r \geq 0$, let $(A,(\alpha, \beta))$ be a solution to (1.20) for the $\operatorname{Spin}^{\mathbb{C}}$ structure in (1.14). Then

$$
2 \pi[\omega] \cdot c_{1}(E)-\frac{z}{r} \leq\left.\frac{r}{4} \int_{X}|1-| \alpha\right|^{2} \mid \leq 2 \pi[\omega] \cdot c_{1}(E)+\frac{z}{r} .
$$

Here $c_{1}(E)$ is the first Chern class of the bundle $E$, and $[\omega]$ is the cohomology class of the symplectic form $\omega$. Also, - signifies the cup-product pairing on cohomology.

Proof of Lemma 2.6. The cup product pairing $c_{1}(E) \cdot[\omega]$ is obtained by integrating over $X$ the 4 -form $\frac{i}{2 \pi} F_{a} \wedge \omega$. This is equal to $\frac{r}{8 \pi}$ times the integral over $\omega$ of $\left(1-|\alpha|^{2}+|\beta|^{2}\right)$ because of (1.20). With this understood, the lemma then follows using Propositions 2.1 and 2.3.

With this lemma understood, it follows that for $r$ sufficiently large, (2.22) has a unique solution $q$, and the maximum principle (with (2.21) and (2.22)) implies that $s-q_{0}-q$ cannot have a positive maximum. That is,

$$
\left|P_{-} F_{a}\right| \leq \frac{r}{4 \sqrt{2}}\left(1+\frac{1}{r} \kappa_{1}\right) \cdot\left(1-|\alpha|^{2}\right)+\kappa_{4}+q
$$

everywhere on $X$. Here, $\kappa_{4}$ is a constant which is independent of $r$ and of the solution $(A,(\alpha, \beta))$.

Step 4. This step completes the proof of Proposition 2.4 under the assumption (Lemma 2.7, below) of a specific bound on the solution $q$ to (2.24). Here is Lemma 2.7:

Lemma 2.7. Fix a complex line bundle $E \rightarrow X$. There are constants $z$ and $z^{\prime}$ that are determined by $c_{1}(E)$ and by the Riemannian metric on $X$ and which have the following significance: Let $r \geq z$, and let $(A,(\alpha, \beta))$ be a solution to (1.20). Let $q$ satisfy (2.21). Then

$$
q \leq z \cdot \sup \left(\left|P_{-} F_{a}\right|\right)\left(\frac{\left(1-|\alpha|^{2}\right)}{r^{1 / 2}}+\frac{z^{\prime}}{r}\right) .
$$

This lemma is proved below. With the lemma given, here is how to complete the proof of Proposition 2.4: First, substitute (2.24) into (2.23) and observe that for $r>z$ (here, $z$ depends only on $c_{1}(E)$ and the metric), the resulting equation bounds $\left|P_{-} F_{a}\right|$ uniformly by a multiple of $r$. (The multiple is $\frac{r}{4 \sqrt{2}}\left(1+\mathcal{O}\left(r^{-1 / 2}\right)\right)$ for $r$ large.) Then, insert this bound for $\left|P_{-} F_{a}\right|$ back into (2.24) and insert the latter into (2.23) to obtain the claimed estimates in Proposition 2.4.

Step 5. This step contains the

Proof of Lemma 2.7. To begin, use $R$ to denote the supremum over $X$ of $|\mathcal{R}|$ and then introduce

$$
q_{1}=q-4 \cdot \frac{R}{r} \sup \left|P_{-} F_{a}\right| .
$$

Then $q_{1}$ obeys the differential inequality

$$
\frac{1}{2} d^{*} d q_{1}+\frac{r}{4}|\alpha|^{2} q_{1} \leq R \cdot \sup \left(\left|P_{-} F_{a}\right|\right) \cdot\left(1-|\alpha|^{2}\right) .
$$

The function $q_{1}$ will be estimated using the maximum principle as applied to $q_{1}-v$, where $v$ is a function which will now be specified. The construction of the 
function $v$ occupies the following digression: To start the digression, remark that Propositions 2.1 and 2.3 plus Lemma 2.2 can be used to find a constant $\delta>0$ (independent of $r$ and $(A,(\alpha, \beta))$ for which the function

$$
v_{1} \equiv 1-|\alpha|^{2}+\frac{\delta}{r}-|\beta|^{2}
$$

has the following properties when $r \geq \kappa_{1}$ :

$$
\begin{aligned}
& \text { (1) } v_{1} \geq \frac{\delta}{2 r} . \\
& \text { (2) } v_{1} \geq 1-|\alpha|^{2} . \\
& \text { (3) } \frac{1}{2} d^{*} d v_{1}+\frac{r}{4}|\alpha|^{2} v_{1} \geq 0 .
\end{aligned}
$$

With (2.28) understood, introduce $\epsilon \equiv r^{-1 / 2}$ and note that $v_{2} \equiv\left(v_{1}\right)^{1-\epsilon}$ obeys the following differential inequality:

$$
\frac{1}{2} d^{*} d v_{2}+\frac{r}{4}|\alpha|^{2} v_{2} \geq \frac{\sqrt{r}}{4}|\alpha|^{2} v_{2} .
$$

Also, notice that

$$
v_{2} \geq 1-|\alpha|^{2},
$$

because of (2.28)(2) and because $1-|\alpha|^{2} \leq 1$. Thus, (2.29) and (2.30) imply that

$$
\frac{1}{2} d^{*} d v_{2}+\frac{r}{4}|\alpha|^{2} v_{2} \geq \frac{\sqrt{r}}{4}|\alpha|^{2} \cdot\left(1-|\alpha|^{2}\right) .
$$

With $v_{2}$ understood, the function $v$ is given by

$$
v \equiv\left(8 R \cdot r^{-1 / 2} \cdot \sup \left(\left|P_{-} F_{a}\right|\right)+4 \cdot \sup \left(q_{1}\right)\right) \cdot v_{2} .
$$

End the digression.

Here are the relevant properties of $v$ : First, $v \geq q_{1}$ at points where $|\alpha|^{2}<1 / 2$; and second, where $|\alpha|^{2} \geq 1 / 2$, the function $v$ obeys the differential inequality

$$
\frac{1}{2} d^{*} d v+\frac{r}{4}|\alpha|^{2} v \geq R \cdot \sup \left(\left|P_{-} F_{a}\right|\right) \cdot\left(1-|\alpha|^{2}\right) .
$$

Thus, the maximum principle applied to $q_{1}-v$ where $|\alpha|^{2} \geq 1 / 2$ proves (using $(2.26)$ and $(2.33))$ that

$$
q_{1} \leq\left(8 R \cdot r^{-1 / 2} \cdot \sup \left(\left|P_{-} F_{a}\right|\right)+4 \cdot \sup \left(q_{1}\right)\right) \cdot v_{2}
$$

everywhere if $r$ is sufficiently large. (This bound on $r$ is independent of the data $(A,(\alpha, \beta))$, although it may depend on $c_{1}(E)$.)

Thus, (2.34) describes $q_{1}$ everywhere once the supremum of $q_{1}$ is estimated. With Lemma 2.6 in hand, the supremum of $q_{1}$ can be estimated directly from (2.26) using the standard properties of the Green's function for the Laplacian. In particular,

$$
q_{1} \leq \frac{z \cdot R \cdot \sup \left(\mid P_{-} F_{a}\right)}{r^{1 / 2}} .
$$

Here, $z$ is independent of $r$ and of $(A,(\alpha, \beta))$ but $z$ does depend on $c_{1}(E)$. (Here is a sketch of the derivation of (2.35): To start, one can drop the second term on the left side of (2.26). Then, use the known singular behavior of the Green's function for $d^{*} d$ to bound $q_{1}$ from above by $z \cdot \sup \left(\left|P_{-} F_{a}\right|\right) \cdot\left(\rho^{-2}\left\|1-|\alpha|^{2}\right\|_{L^{1}}+\rho^{2}\right)$, where $\rho>0$ is arbitrary. The constant $z$ here depends only on the Riemannian metric. Since the $L^{1}$ norm of $\left(1-|\alpha|^{2}\right)$ is bounded from above by a multiple of $r^{-1}$ from 
Propositions 2.1 and 2.3 plus Lemma 2.6, the preceding bound is minimized by taking $\rho=r^{-1 / 4}$. This gives the bound in (2.35).)

This estimate from (2.35) should now be plugged back into (2.34) and (2.25) to give a pointwise bound for $q$. Note that the resulting expression is that which is claimed in Lemma 2.7 except that the function $\left(1-|\alpha|^{2}\right)$ in $(2.24)$ has been replaced by the function $v_{2}$. However, $v_{2}$ obeys

$$
v_{2} \leq 2 \cdot\left(1-|\alpha|^{2}\right)+\frac{z_{0}}{r},
$$

which follows from $(2.28)(1)$. Thus the estimate in Lemma 2.7 is proved.

(e) Estimates on the covariant derivatives of $(\alpha, \beta)$. The covariant derivatives of $\alpha$ and $\beta$ are bounded by

Proposition 2.8. Let $E \rightarrow X$ be a complex line bundle. There are constants $z$ and $z^{\prime}$ that are determined by $c_{1}(E)$ and by the Riemannian metric on $X$ and which have the following significance: Let $r \geq 1$ and suppose that $(A,(\alpha, \beta))$ is a solution to (1.20) for the $\mathrm{Spin}^{\mathbb{C}}$ structure with $S_{+}$as in (1.14). Then

$$
\left|\nabla_{a} \alpha\right|^{2}+r \cdot\left|\nabla_{A}^{\prime} \beta\right|^{2} \leq z^{\prime} \cdot r\left(1-|\alpha|^{2}\right)+z .
$$

The remainder of this subsection is devoted to the

Proof of Proposition 2.8. The starting point is (2.1).

Step 1. Project (2.1) onto the $E$ summand in (1.14) and then act on both sides of the resulting equation by $\nabla_{a}$. Commute covariant derivatives where necessary to get an equation of the form $\nabla_{a}^{*} \nabla_{a}\left(\nabla_{a} \alpha\right)=$ something. Then take the inner product of the resulting equation with $\nabla_{a} \alpha$ to obtain the following differential inequality:

$$
\begin{aligned}
\frac{1}{2} d^{*} d \mid & \left.\nabla_{a} \alpha\right|^{2}+\left|\nabla_{a} \nabla_{a} \alpha\right|^{2}+\frac{r}{4}|\alpha|^{2}\left|\nabla_{a} \alpha\right|^{2} \\
\leq & z \cdot\left(r \cdot\left(1-|\alpha|^{2}\right)+1\right)\left|\nabla_{a} \alpha\right|^{2} \\
& +z \cdot\left|\nabla_{a} \alpha\right|\left((1+r|\beta|) \cdot\left|\nabla_{A}^{\prime} \beta\right|+\left(r|\alpha|^{2}+1\right)|\beta|+\left|\nabla_{A}^{\prime} \nabla_{A}^{\prime} \beta\right|\right) .
\end{aligned}
$$

Proposition 2.4 was used in deriving (2.38) in order to bound terms where $F_{a}$ appears from commutators of covariant derivatives. The commuting of covariant derivatives also produces a term with $d^{*} F_{a}$; the latter is expressed in terms of $\alpha, \beta$ and their covariant derivatives using (2.16) and the fact that $d^{*} F_{a}=2 d\left(P_{+} F_{a}\right)$. Furthermore, certain manifestly positive terms were discarded from the left-hand side of (2.38) (i.e. multiples of $\left.\left.|d| \alpha\right|^{2}\right|^{2}$ and $|\beta|^{2}\left|\nabla_{a} \alpha\right|^{2}$ ). In (2.38), the constant $z$ is independent of $r$ and of the solution $(A,(\alpha, \beta))$. An application of the triangle inequality (and Propositions 2.1 and 2.3) to the right side of (2.38) gives the following, less cumbersome inequality:

$$
\begin{aligned}
& \frac{1}{2} d^{*} d\left|\nabla_{a} \alpha\right|^{2}+\left|\nabla_{a} \nabla_{a} \alpha\right|^{2}+\frac{r}{4}|\alpha|^{2}\left|\nabla_{a} \alpha\right|^{2} \\
& \quad \leq z_{1} \cdot r \cdot\left|\nabla_{a} \alpha\right|^{2}+z_{1} \cdot r|\beta|^{2}+z_{1} \cdot\left|\nabla_{A}^{\prime} \beta\right|^{2}+\frac{1}{r}\left|\nabla_{A}^{\prime} \nabla_{A}^{\prime} \beta\right|^{2}
\end{aligned}
$$

Here, $z_{1}$ is a constant which is independent of $r$ and of $(A,(\alpha, \beta))$. 
Step 2. Now project (2.1) onto the $K^{-1} \otimes E$ summand in (1.14) and then act on both sides of the resulting equation by $\nabla_{A}^{\prime}$. Then take the inner product of the resulting equation with $\nabla_{A}^{\prime} \beta$ to obtain the following differential inequality:

$$
\begin{aligned}
\frac{1}{2} d^{*} d \mid & \left.\nabla_{A}^{\prime} \beta\right|^{2}+\left|\nabla_{A}^{\prime} \nabla_{A}^{\prime} \beta\right|^{2}+\frac{r}{4}|\alpha|^{2}\left|\nabla_{A}^{\prime} \beta\right|^{2} \\
\leq & z_{2} \cdot\left(r \cdot\left(1-|\alpha|^{2}\right)+1\right) \cdot\left|\nabla_{A}^{\prime} \beta\right|^{2} \\
& +z_{2} \cdot\left|\nabla_{A}^{\prime} \beta\right|\left((1+r|\alpha||\beta|) \cdot\left|\nabla_{a} \alpha\right|+r|\alpha||\beta|^{2}+\left|\nabla_{a} \nabla_{a} \alpha\right|\right) .
\end{aligned}
$$

In deriving (2.40), certain manifestly positive terms were discarded from the left side (i.e. multiples of $\left.\left.|d| \beta\right|^{2}\right|^{2}$ and $|\beta|^{2}\left|\nabla_{A}^{\prime} \beta\right|^{2}$ ). And, Proposition 2.4 and also (2.16) were used to bound the terms which arise from commuting covariant derivatives. As usual, in (2.40), the constant $z$ is independent of $r$ and $(A,(\alpha, \beta))$. An application of the triangle inequality (and Propositions 2.1 and 2.3) to the right side of (2.40) gives the following inequality:

$$
\begin{aligned}
& \frac{1}{2} d^{*} d\left|\nabla_{A}^{\prime} \beta\right|^{2}+\left|\nabla_{A}^{\prime} \nabla_{A}^{\prime} \beta\right|^{2}+\frac{r}{4}|\alpha|^{2}\left|\nabla_{A}^{\prime} \beta\right|^{2} \\
& \quad \leq z_{3} \cdot r \cdot\left|\nabla_{a}^{\prime} \beta\right|^{2}+z_{3}|\beta|^{2}+z_{3} \cdot\left|\nabla_{a} \alpha\right|^{2}+\frac{1}{r}\left|\nabla_{a} \nabla_{a} \alpha\right|^{2} .
\end{aligned}
$$

Here, as usual, $z_{3}$ is independent of $r$ and $(A,(\alpha, \beta))$.

Step 3. This third step consists of adding (2.39) to $r$ times (2.41). The result (after a straightforward rearrangement, and after the discarding of certain manifestly positive terms from the left side) is the following inequality for $y \equiv\left(\left|\nabla_{a} \alpha\right|^{2}+\right.$ $\left.r\left|\nabla_{A}^{\prime} \beta\right|^{2}\right)$ :

$$
\frac{1}{2} d^{*} d y+\frac{r}{4}|\alpha|^{2} y \leq z_{4} \cdot r \cdot\left(\left|\nabla_{a} \alpha\right|^{2}+r\left|\nabla_{A}^{\prime} \beta\right|^{2}\right)+z_{4} \cdot r \cdot|\beta|^{2} .
$$

Here, $z_{4}$ is a new constant which is independent of $r$ and $(A,(\alpha, \beta))$.

With (2.42) understood, introduce the function $u$ from (2.5) using the values $\zeta \equiv \frac{r}{\kappa_{1} \kappa_{2}}$ and with $\delta \equiv 1$. It then follows from (2.6) that there exists a constant $z_{5}$ which is independent of both $r$ and $(A,(\alpha, \beta))$, and is such that

$$
\frac{1}{2} d^{*} d\left(y-z_{5} \cdot r \cdot u\right)+\frac{r}{4}|\alpha|^{2}\left(y-z_{5} \cdot r \cdot u\right) \leq 0 .
$$

Hence, the maximum principle implies that $y \leq z_{5} \cdot r \cdot u$ which is the assertion of Proposition 2.8.

\section{The MONOTONICITY FORMULA}

Fix a $\operatorname{Spin}^{\mathbb{C}}$ structure as in (1.14) and suppose that $(A,(\alpha, \beta))$ is a solution to (1.20) for some large $r$. The purpose of this section is to derive a lower bound for the amount of "energy" of the $\alpha$ part of the spinor in a ball when $\alpha$ is zero at the ball's center. This lower bound will be seen to grow as the square of the radius of the ball. This growth rate estimate plays a key role in the subsequent construction of pseudo-holomorphic curves from sequences of $\alpha$ zero sets.

The growth rate estimate is stated in Proposition 3.1, below. The key to proving Proposition 3.1 is the formula in Proposition 3.2 for the rate of change of the energy in a ball as a function of the radius of the ball. (Such formulae are sometimes called "monotonicity estimates".) 
Subsections 3a-c are concerned with Propositions 3.1 and 3.2 and their proofs. The last subsection below offers a refinement (Proposition 3.4) of the estimate in Proposition 2.4 for $F_{a}$.

(a) Local energy. Fix a complex line bundle $E \rightarrow X$. Then, fix $r>0$ and a solution $(A,(\alpha, \beta))$ of $(1.20)$ using the Spin ${ }^{\mathbb{C}}$ structure in (1.14). Let $U \subset X$ be an open set. Define the energy $\mathcal{E}_{U} \equiv \mathcal{E}_{U}(\alpha)$ over $U$ to be equal to the integral over $B$ of

$$
\left.\mathcal{E}_{U} \equiv \frac{r}{4} \int_{U}|1-| \alpha\right|^{2} \mid .
$$

Lemma 2.6 asserts that this energy cannot be too big.

(b) Monotonicity. Fix the $\operatorname{Spin}^{\mathbb{C}}$ structure on $X$ as in (1.14). Fix a point $x \in X$ and let $B$ denote the geodesic ball of radius $s>0$ with center $x$. Let $r>0$ be given, as well as a solution $(A,(\alpha, \beta))$ to (1.20). The following proposition details the behavior of $\mathcal{E}_{B}$ as a function of the radius $s$ of $B$. It has two parts. The first part gives upper bounds for the size of $\mathcal{E}_{B}$; and the second part gives lower bounds under the assumption that $|\alpha(x)|$ is small.

Proposition 3.1. Fix a complex line bundle $E \rightarrow X$. There is a constant $z \geq 1$ that is determined by $c_{1}(E)$ and the Riemannian metric and which has the following significance: Fix $r \geq z$ as well as a solution $(A,(\alpha, \beta))$ to $(1.20)$ for the Spin $^{\mathbb{C}}$ structure in (1.14). Let $B \subset X$ be a geodesic ball with center $x$. Let $s$ denote the radius of $B$ and require $\frac{1}{z} \geq s \geq \frac{1}{2 r^{1 / 2}}$. Then

(1) $\mathcal{E}_{B} \leq z \cdot s^{2}$.

(2) If $|\alpha(x)|<1 / 2$, then

$$
\mathcal{E}_{B} \geq \frac{1}{z+1} s^{2}
$$

The strategy for proving Proposition 3.1 is to derive an inequality involving $\mathcal{E}_{B}$ and $\frac{d}{d s} \mathcal{E}_{B}$ which can be integrated to yield (3.2). This inequality is stated as

Proposition 3.2. Fix a complex line bundle $E \rightarrow X$. There is a constant $z \geq 1$ that is determined by $c_{1}(E)$ and the Riemannian metric and which has the following significance: Specify $r \geq z$ as well as a solution $(A,(\alpha, \beta))$ to $(1.20)$ for the $\operatorname{Spin}^{\mathbb{C}}$ structure in (1.14). Let $B \subset X$ be a geodesic ball with center $x$ which is such that $\alpha(x)<\frac{1}{2}$. Let $s$ denote the radius of $B$ and require that $s \geq \frac{1}{r^{1 / 2}}$ but $s<\frac{1}{z}$. Then

$$
\mathcal{E}_{B} \leq \frac{s}{2}(1+z s) \cdot\left(1+\frac{z}{r^{1 / 2}}\right) \frac{d}{d s} \mathcal{E}_{B}+z \cdot s^{4} .
$$

The remainder of this subsection proves Proposition 3.1 under the assumption of the validity of (3.3). The next subsection contains the derivation of (3.3).

Proof of Proposition 3.1. Before beginning, it proves useful to make a convention concerning constants which appear in the formulae below: Any constant which is labeled by $z$ (with or without a numerical subscript, such as $z_{0}$; and with or without a prime, such as $z^{\prime}$ ) should be assumed to be defined independent of the parameter $r$ and of the data $(A,(\alpha, \beta))$. Unless stated to the contrary, such constants should be assumed to depend only on $c_{1}(E)$, the Riemannian metric and the symplectic form $\omega$.

There are three steps to the proof of Proposition 3.1. 
Step 1. This step integrates (3.3). For this purpose, let

$$
f(s) \equiv-2 \cdot\left(1+\frac{z}{r^{1 / 2}}\right)^{-1} \cdot \ln \left(\frac{s}{(1+z s)}\right) .
$$

With $f$ understood, then (3.4) reads

$$
\frac{d}{d s}\left(e^{f} \mathcal{E}_{B}\right) \geq-z \cdot s^{3} \cdot e^{f}
$$

This last equation can be integrated to read

$$
\left.\mathcal{E}_{B}\right|_{s} \geq \frac{1}{z^{\prime}} \frac{s^{2}}{s_{0}^{2}}\left(\left.\mathcal{E}_{B}\right|_{s_{0}}-z^{\prime} \cdot s_{0}^{2} s^{2}\right)
$$

Here, $s_{0} \geq r^{-1 / 2}$. Note that in deriving the right side in (3.6), use has been made of the fact that as $r$ gets large, the function $r^{-1 / 2} \ln (r)$ tends uniformly to zero. To be more precise here, (3.6) uses the fact that

$$
\frac{1}{z_{1} s^{2}} \leq e^{f} \leq \frac{z_{1}}{s^{2}}
$$

where $z_{1}$ is independent of $r$ and $s$ as long as $r^{-1 / 2}<s<\rho_{0} / 100$.

To complete Step 2 of the proof, suppose that $s_{0}$ has been given with $r^{-1 / 2}<$ $s_{0}<\rho_{0} / 100$ and with

$$
\left.\mathcal{E}_{B}\right|_{s=s_{0}} \geq \zeta \cdot s_{0}^{2}
$$

Under this assumption, (3.6) implies that

$$
\left.\mathcal{E}_{B}\right|_{s} \geq \frac{\zeta s^{2}}{z^{\prime \prime}} \text { when }\left(\frac{\zeta}{2 z^{\prime}}\right)^{1 / 2} \geq s \geq s_{0} .
$$

Step 2 . This step proves assertion (1) by remarking that $\mathcal{E}_{X}$ is uniformly bounded from below by Lemma 2.6 , but also $\mathcal{E}_{X} \geq\left.\mathcal{E}_{B}\right|_{s=\rho_{0} / 100}$. This last quantity is estimated from below by (3.9), and so Lemma 2.6 gives an upper bound for $\zeta$ to prove assertion (1) of Proposition 3.1.

Step 3. This step bounds $\mathcal{E}_{B}$ from below when $s=s_{0} \equiv r^{-1 / 2}$ and $|\alpha|(x)<1 / 2$. Here is the bound: When $s_{0}=r^{-1 / 2}$, then

$$
\left.\mathcal{E}_{B}\right|_{s=s_{0}}>\frac{1}{z \cdot r}
$$

Given this bound, assertion (2) of Proposition 3.1 follows from (3.9). To prove (3.10), note that the assumption that $|\alpha(x)|<1 / 2$ and then Proposition 2.8 imply that $|\alpha(y)|<3 / 4$ when $\operatorname{dist}(y, x)<\frac{1}{z_{1} \cdot r^{1 / 2}}$. With this understood, it follows that (3.1) is at least

$$
\frac{r}{100} \times\left(\text { Volume of the ball of radius } \frac{1}{z_{1} \cdot r^{1 / 2}}\right)
$$

when $s=r^{-1 / 2}$. 
(c) Proof of Proposition 3.2. First, use the same convention with constants labeled by $z$ as in the proof of Proposition 3.1.

The proof of Proposition 3.2 requires four steps. But, the key to the proof is an integration by parts formula which is derived in Step 1. The integration by parts formula is then reinterpreted in the subsequent steps.

Step 1 . The derivation of the integration by parts formula starts with the fact that the symplectic form $\omega$ is exact in $B$ and so can be written $\omega=d \theta$ where $\theta$ is a smooth 1-form in B. Thus, with (3.14), one has

$$
\int_{B} \omega \wedge i \cdot F_{a}=\int_{\partial B} \theta \wedge i \cdot F_{a} .
$$

Step 2. This step rewrites the left side of (3.12) so as to produce a bound on $\mathcal{E}_{B}$. The point here is that the integrand on the left side of (3.12) can be related to the integrand in (3.1) for $\mathcal{E}_{B}$. Indeed, using the curvature equation in (1.20), one finds the second term in the integrand on the left side of (3.12) equal to

$$
\frac{r}{4}\left(1-|\alpha|^{2}+|\beta|^{2}\right) .
$$

Because (2.11) implies the lower bound

$$
-\frac{z_{1}}{r^{2}} \leq\left(1-|\alpha|^{2}\right),
$$

the expression in (3.13) is no smaller than

$$
\left.\frac{r}{4}|1-| \alpha\right|^{2} \mid-\frac{z_{2}}{r} .
$$

Insert the bound from (3.15) into the left side of (3.12) to arrive, finally, at the inequality

$$
\mathcal{E}_{B}-\frac{z_{3} \cdot s^{4}}{r} \leq \int_{\partial B} \theta \wedge i \cdot F_{a} .
$$

Notice that the inequality in (3.16) bounds $\mathcal{E}_{B}$ using an integral over $\partial B$.

Step 3. This step comprises a three-part digression concerning the possibilities for the 1 -form $\theta$ in (3.16).

For part 1 of the digression, recall that there are no local invariants for symplectic forms. This means that there exists some number $\rho>0$ such that there is a "symplectic" coordinate system on the radius $\rho$ ball about any point in $X$. Such a coordinate system is characterized by the fact that it pulls $\omega$ back to $\mathbb{R}^{4}$ as the standard symplectic form

$$
\omega_{0}=d y^{1} \wedge d y^{2}+d y^{3} \wedge d y^{4} .
$$

Here, $\left(y^{1}, y^{2}, y^{3}, y^{4}\right)$ are standard Euclidean coordinates.

Now, there is some flexibility in choosing a symplectic coordinate system; and, in particular, one can choose the symplectic coordinate system so that the metric, $g$, on $X$ pulls back to $\mathbb{R}^{4}$ to obey

$$
\begin{aligned}
& \text { (1) }\left|g-g_{E}\right| \leq z_{0} \cdot|y| \text {. } \\
& \text { (2) }|\partial g| \leq z_{0} .
\end{aligned}
$$

Here, $g_{E}$ denotes the Euclidean metric, and $\partial g$ denotes the tensor of partial derivatives (with respect to the Euclidean coordinates) of the components of the metric 
$g$. The constant $z_{0}$ in (3.18) can be taken to be independent of the given point on $X$.

For part 2 of the digression, remark that the form $\omega_{0}$ on $\mathbb{R}^{4}$ can be written as $\omega_{0}=d \theta_{0}$, where

$$
\theta_{0}=\frac{1}{2}\left(x^{1} d x^{2}-x^{2} d x^{1}+x^{3} d x^{4}-x^{4} d x^{3}\right) .
$$

This 1-form $\theta_{0}$ has some useful properties which are described below:

Lemma 3.3. Let $s>0$ and let $S_{s}$ denote the 3 -sphere in $\mathbb{R}^{4}$ of radius $s$ and center 0 . Endow $S_{s}$ with the induced metric from its embedding $\iota: S_{s} \rightarrow \mathbb{R}^{4}$ and then let * : $T^{*} S_{s} \rightarrow \Lambda^{2} T^{*} S_{s}$ denote the induced metric's Hodge star. Then

$$
\left|\iota^{*} \theta_{0}\right|=\frac{s}{2}
$$

Proof of Lemma 3.3. This is an exercise.

For part 3 of the digression, note that one can find (using part 1 of the digression) a number $\rho_{0}>0$, which depends only on the metric on $X$ and on $\omega$ and which has the following significance: If $s<\rho_{0} / 100$, then the ball $B$ of radius $s$ and center $x$ lies in the symplectic coordinate chart about $x$. Use these coordinates to think of $B$ as a subset in $\mathbb{R}^{4}$. Note that $B$ is a ball as defined with the metric $g$, but it is not necessarily a Euclidean ball.

In any event, let $\iota: \partial B \rightarrow \mathbb{R}^{4}$ denote the inclusion. Then, the form $\theta$ which appears on the right side of $(3.16)$ can be taken to be $\iota^{*} \theta_{0}$. Because of (3.18) and Lemma 3.3, this form obeys

$$
|| \theta\left|-\frac{s}{2}\right| \leq z_{5} \cdot s^{2}
$$

(Because of (3.18), the norms here can be taken either with the Euclidean metric or with the metric $g$.) The constant $z_{5}$ in (3.20) can be taken independent of $s$ as long as $s<\frac{\rho}{100}$.

Step 4. This step rewrites the right side of (3.16) in terms of the derivative $\frac{d}{d s} \mathcal{E}_{B}$. To begin, observe that

$$
\left|\theta \wedge \iota^{*} F_{a}\right| \leq \frac{s}{2}\left(1+z_{4} s\right) \cdot\left(\left|\iota^{*} P_{-} F_{a}\right|+\left|\iota^{*} P_{+} F_{a}\right|\right)
$$

Use the fact that

$$
\left|\iota^{*} P_{ \pm} F_{a}\right|=\frac{1}{\sqrt{2}}\left|P_{ \pm} F_{a}\right|
$$

to invoke (2.12) and (2.13) in order to simplify the right side of (3.21) so that the result reads

$$
\left|\theta \wedge \iota^{*} F_{a}\right| \leq \frac{s}{2}\left(1+z_{5} s\right) \cdot\left(1+\frac{z_{5}}{r^{1 / 2}}\right) \cdot\left(\left.\frac{r}{4}|1-| \alpha\right|^{2} \mid+z_{5}\right) .
$$

Now, plug (3.23) into the right side of (3.16) to find

$$
\mathcal{E}_{B} \leq\left.\frac{s}{2}\left(1+z_{5} s\right) \cdot\left(1+\frac{z_{6}}{r^{1 / 2}}\right) \frac{r}{4} \int_{\partial B}|1-| \alpha\right|^{2} \mid+z_{6} \cdot s^{4} .
$$

The final chapter of the story rewrites (3.24) using the observation

$$
\frac{d}{d s} \mathcal{E}_{B}=\left.\frac{r}{4} \int_{\partial B}|1-| \alpha\right|^{2} \mid .
$$

This last identity with (3.24) gives the proposition. 
(d) A refined estimate of $P_{-} F_{a}$. The monotonicity formula in Proposition 3.2 has an immediate application towards refining the upper bound in Proposition 2.4 for the size of $P_{-} F_{a}$. Here is the refined bound:

Proposition 3.4. Fix a complex line bundle $E \rightarrow X$. There is a constant $z \geq 1$ that is determined by $c_{1}(E)$ and the Riemannian metric and which has the following significance: Let $r \geq 1$ and suppose that $(A,(\alpha, \beta))$ is a solution to (1.20) for the Spin $^{\mathbb{C}}$ structure with $S_{+}$as in (1.14). Then

$$
\left|P_{ \pm} F_{a}\right| \leq \frac{r}{4 \sqrt{2}}\left(1-|\alpha|^{2}\right)+z .
$$

Remark that the refinement in (3.26) over the estimate in (2.13) is the absence of the factor of $r^{1 / 2}$. The remainder of this subsection is occupied with the

Proof of Proposition 3.4. To begin, remark that the asserted inequality for $\left|P_{+} F_{a}\right|$ follows directly from (2.12) and Proposition 2.3. Thus, the task is to prove the bound for $\left|P_{-} F_{a}\right|$. Reviewing the proof of Proposition 2.4, one finds that the factor of $r^{1 / 2}$ in (2.13) enters through the estimate for the function $q$ in (2.25). With this understood, the proof of Proposition 3.4 is obtained by finding a bound for the function $q$ by a constant (which depends only on $c_{1}(E)$ and the Riemannian metric). This gives the required bound for $P_{-} F_{a}$ via (2.23). In summary, the task is to bound $q$ by an $r$-independent constant.

To begin the task, use the fact that $\left|P_{-} F_{a}\right|$ is a priori bounded in Proposition 2.4 by some constant times $r$ to argue that $q$ satisfies the differential inequality

$$
\frac{1}{2} d^{*} d q+\frac{r}{4}|\alpha|^{2} q \leq z_{1} \cdot r .
$$

Here, $z_{1}$ is determined by the Riemannian metric and by $c_{1}(E)$, but it is independent of $r$ and of $(A,(\alpha, \beta))$. The following lemma is the key to the refined bounds for $q$ :

Lemma 3.5. Fix a complex line bundle $E \rightarrow X$. There is a constant $z_{0} \geq 1$ that is determined by $c_{1}(E)$ and the Riemannian metric and which has the following significance: Let $r \geq 1$ and let $(A,(\alpha, \beta))$ be a solution to (1.20) for the $\operatorname{Spin}^{\mathbb{C}}$ structure with $S_{+}$as in (1.14). There is a smooth function $u$ on $X$ which obeys

(1) $|u| \leq z_{0}$.

(2) $\frac{1}{2} d^{*} d u \geq r$ where $|\alpha|<\frac{1}{2}$.

(3) $\left|d^{*} d u\right| \leq z_{0} \cdot r$.

This lemma is proved below. Accept its validity for now to continue with the proof of Proposition 3.4 as follows: Take the constant $z_{1}$ from (3.27) and consider that the function $q-z_{1} \cdot u$ obeys the differential inequality

$$
\frac{1}{2} d^{*} d\left(q-z_{1} \cdot u\right)+\frac{r}{4}|\alpha|^{2}\left(q-z_{1} \cdot u\right) \leq z_{2} \cdot r \cdot|\alpha|^{2} .
$$

This follows from assertions (2) and (3) of Lemma 3.5. (Here, $z_{2}$ is independent of $r$ and of $(A,(\alpha, \beta))$; it depends only on $c_{1}(E)$ and the Riemannian metric.) Next, apply the maximum principle to the function $\left(q-z_{1} \cdot u-4 z_{2}\right)$ to conclude that this function is either zero or everywhere negative. Thus,

$$
q \leq z_{1} \cdot u+4 z_{2} \leq z_{3},
$$

which is a consequence of assertion (1) of Lemma 3.5. As remarked, this bound for $q$ plus (2.23) yields Proposition 3.4. 
Proof of Lemma 3.5. In the proof, strictly positive constants which depend on $c_{1}(E)$ and the Riemannian metric, but do not depend on $r$ nor on $(A,(\alpha, \beta))$ will be denoted by $z$, with or without numerical subscripts. Usually, this fact will be left unsaid.

The proof of Lemma 3.5 is divided into five steps.

Step 1. The first observation is

Lemma 3.6. Fix a complex line bundle $E \rightarrow X$. There is a constant $z_{1} \geq 1$ that is determined by $c_{1}(E)$ and the Riemannian metric and which has the following significance: Suppose that $r \geq z_{1}$ and that $(A,(\alpha, \beta))$ is a solution of $(1.20)$ with the $\operatorname{Spin}^{\mathbb{C}}$ structure from (1.14). Fix $\rho \in\left(r^{-1 / 2}, \rho_{0} / 100\right)$. Then, the set $V$ where $|\alpha|<\frac{1}{2}$ can be covered by a set $\left\{B_{i}\right\}$ of no more than $\frac{z_{1}}{\rho^{2}}$ balls of radius $\rho$ whose centers are in $V$. Furthermore, if $B_{i}^{\prime}$ is the ball with the same center as $B_{i}$ but with radius $\frac{\rho}{2}$, then the balls in the set $\left\{B_{i}^{\prime}\right\}$ are disjoint.

Proof of Lemma 3.6. If $B$ is a ball of radius $\frac{1}{2} \rho \geq \frac{1}{2} r^{-1 / 2}$ and center $x \in V$, then according to Proposition 3.1, $\mathcal{E}_{B} \geq \frac{1}{z+1} \rho^{2}$. This fact with Lemma 2.6 implies that there are no more than $\frac{z^{\prime}}{\rho^{2}}$ such balls which are mutually disjoint. Let $\left\{B_{i}^{\prime}\right\}$ be a maximal (in number) set of balls of radius $\rho / 2$ with centers where $|\alpha|<\frac{1}{2}$. Then the set of concentric balls $\left\{B_{i}\right\}$ of radius $\rho$ covers the set where $|\alpha|<\frac{1}{2}$.

Step 2. Take $\rho=r^{-1 / 2}$ and invoke Lemma 3.6. With the balls $\left\{B_{i}\right\}$ specified by the lemma, the next step is to prove that there are many points in $X-V$ which are not too far from any $B_{i}$. The following lemma makes this precise:

Lemma 3.7. Given $\lambda>0$, there exists a constant $z \equiv z(\lambda)$ which depends only on $c_{1}(E)$ and the metric, not on $r$ nor $(A,(\alpha, \beta))$, with the following significance: Let $V^{\prime} \subset X$ denote the set of points where $|\alpha| \leq \frac{3}{4}$. (Thus, $V \subset V^{\prime}$.) Take any $B$ from the set $\left\{B_{i}\right\}$. Let $\mathfrak{B}$ denote the ball of radius $\frac{z}{r^{1 / 2}}$ whose center is the same as that of $B$. Then

$$
\operatorname{volume}\left(\left(X-V^{\prime}\right) \cap \mathfrak{B}\right) \geq \lambda \cdot \operatorname{volume}(B) .
$$

Proof of Lemma 3.7. The proof uses the monotonicity formula in Proposition 3.1. Given $\lambda$, suppose there exists $\zeta>1$ and a ball $\mathfrak{B}$ of radius $\frac{\zeta}{r^{1 / 2}}$ and center where $|\alpha|<\frac{1}{2}$ with the property that volume $\left(V^{\prime} \cap \mathfrak{B}\right) \geq \operatorname{volume}(\mathfrak{B})-\frac{z_{1} \lambda}{r^{2}}$. In particular, if $\zeta>z_{2} \cdot \lambda^{1 / 4}$, then volume $\left(V^{\prime} \cap \mathfrak{B}\right)$ can be assumed larger than $\frac{\zeta^{4}}{2 r^{2}}$. Thus, $\mathcal{E}_{\mathfrak{B}}$ is bounded from below by $\frac{z_{3} \zeta^{4}}{r}$. Now invoke Assertion (1) of Proposition 3.1 to bound $\zeta$.

Step 3 . With the preceding understood, take $\lambda=1$ in Lemma 3.7 and let $z_{0} \equiv z(1)$ be the corresponding constant provided by Lemma 3.7. For each $B_{i}$ from Lemma 3.6 's set of balls, let $\mathfrak{B}_{i}$ denote the ball of radius $\frac{z_{0}}{r^{1 / 2}}$ whose center is the same as that of $B_{i}$. Let $s_{i}$ denote the characteristic function of the ball $B_{i}$ and let $\mathfrak{s}_{i}$ denote the characteristic function of the set $\left(X-V^{\prime}\right) \cap \mathfrak{B}_{i}$. (A characteristic function of a set is equal to one at points in the set and zero at all other points.)

Note that the integrals of either $s_{i}$ or $\mathfrak{s}_{i}$ over $X$ are bounded from above by $\frac{z_{1}}{r^{2}}$ and bounded from below by $\frac{1}{z_{1} r^{2}}$. Thus, there is a constant $\kappa_{i}$ with

$$
\frac{1}{z_{2}}<\kappa_{i}<z_{2}
$$


with the property that $s_{i}-\kappa \mathfrak{s}_{i}$ integrates to zero over $X$. And, there is a unique $C^{1,1}$ function $u_{i}$ on $X$ which satisfies the equation

$$
\frac{1}{2} d^{*} d u_{i}=r \cdot\left(s_{i}-\kappa_{i} \mathfrak{s}_{i}\right) .
$$

(The fact that $u_{i}$ is not smooth here follows from the fact that $s_{i}$ and $\mathfrak{s}_{i}$ are characteristic functions. One could smooth the $s_{i}$ and $\mathfrak{s}_{i}$ arbitrarily close to the boundaries of their domains of definition in order to work with smooth functions. This would make no essential difference in subsequent arguments.)

Step 4 . This step will provide a uniform bound for the function $u_{i}$. The task is facilitated with the introduction of the Green's function $G$ for the operator $d^{*} d$. This $G$ can be taken to have the following properties:

$$
\text { (1) } G \geq 1 \text {. }
$$

$$
\begin{aligned}
& \text { (2) } \frac{1}{\kappa \operatorname{dist}(x, y)^{2}} \leq G(x, y) \leq \frac{\kappa}{\operatorname{dist}(x, y)^{2}}, \\
& \text { (3) }|\nabla G(\cdot, y)|_{x} \leq \frac{\kappa}{\operatorname{dist}(x, y)^{3}} .
\end{aligned}
$$

Here, $\kappa \geq 1$ is a metric dependent constant. (Note that $\operatorname{dist}(\cdot, \cdot)$ is the distance function for the Riemannian metric.)

With $G$ understood, then the function $u_{i}$ solving (3.32) can be taken to be

$$
u_{i}(x) \equiv r \int_{X} G(x, \cdot) \cdot\left(s_{i}-\kappa_{i} \mathfrak{s}_{i}\right) \cdot d \mathrm{vol}
$$

This last equation can be used to estimate $u_{i}$. For this purpose, introduce $x_{i}$ to denote the center of $B_{i}$. First of all,

$$
\left|u_{i}(x)-r \cdot G\left(x, x_{i}\right) \cdot\left(\int_{X}\left(s_{i}-\kappa_{i} \mathfrak{s}_{i}\right) d \mathrm{vol}\right)\right| \leq \frac{z_{3}}{r^{3 / 2} \operatorname{dist}\left(x, x_{i}\right)^{3}}
$$

if $\operatorname{dist}\left(x, x_{i}\right)>\frac{10 z_{0}}{r^{1 / 2}}$. This follows from (3.33)(3) using Taylor's theorem with remainder. Since $\left(s_{i}-\kappa_{i} \mathfrak{s}_{i}\right)$ has integral zero over $X$, the conclusion is that

$$
\left|u_{i}(x)\right| \leq \frac{z_{4}}{r^{3 / 2} \operatorname{dist}\left(x, x_{i}\right)^{3}}
$$

as long as $\operatorname{dist}\left(x, x_{i}\right)>\frac{10 z_{0}}{r^{1 / 2}}$. On the other hand, it follows from (3.33)(2) and (3.40) that

$$
u_{i}(x)<z_{4}, \quad \text { where } \operatorname{dist}\left(x, x_{i}\right) \leq \frac{10 z_{0}}{r^{1 / 2}}
$$

Step 5 . With $\left\{u_{i}\right\}$ understood, define the function $u$ by

$$
u \equiv \sum_{i} u_{i} .
$$

The task now is to verify the properties which are asserted in Lemma 3.5. The verification will proceed in reverse order, so consider property (3) first:

Clearly,

$$
\left|d^{*} d u\right| \leq 2 \sum_{i} r \cdot\left|s_{i}-\kappa_{i} \mathfrak{s}_{i}\right| .
$$

The requisite bound follows from (3.39) with the observation that there are at most $z_{5}$ terms in the sum in (3.39) which can be non-zero at any given point. (This is because each term has support only in the ball $\mathfrak{B}_{i}$ and no more than $z_{5}$ of these 
can mutually intersect because the balls $\left\{B_{i}^{\prime}\right\}$ are mutually disjoint. See Lemma 3.6.)

As for property (2), each $\mathfrak{s}_{i} \equiv 0$ where $|\alpha|<\frac{1}{2}$, so

$$
\frac{1}{2} d^{*} d u=r \cdot \sum_{i} s_{i}
$$

where $|\alpha|<\frac{1}{2}$. And, at $x$ where $|\alpha(x)|<\frac{1}{2}$, the sum on the right side of (3.40) is greater than one, since $s_{i} \equiv 1$ on $B_{i}$ and $\bigcup_{i} B_{i}$ contains all points where $|\alpha|<\frac{1}{2}$.

The last part of the proof of Lemma 3.5 is to verify the asserted bound for the size of $u$. The following lemma is required:

Lemma 3.8. There is a constant $z$ which depends only on $c_{1}(E)$ and the Riemannian metric and which has the following significance: Fix a point $x \in X$. When $n$ is a positive integer, let $B(x, n) \subset X$ denote the ball with center $x$ and radius $\frac{n}{r^{1 / 2}}$. Use $N(n)$ to denote the number of balls from the set $\left\{B_{i}\right\}$ whose centers lie in $B(x, n)$. Then $N(n)<z \cdot n^{2}$.

Proof of Lemma 3.8. Each $B_{i}$ contains the smaller ball $B_{i}^{\prime}$ which contributes at least $\frac{1}{z_{1} r}$ to $\mathcal{E}_{X}$. These $\left\{B_{i}^{\prime}\right\}$ are disjoint, so the contribution from $B(x, n+1)$ to $\mathcal{E}_{X}$ is at least $\frac{N(n)}{z_{1} r}$. Now invoke Assertion 1 of Proposition 3.1 to bound $N(n)$.

With this lemma in hand, fix $x \in X$ and consider estimating $u(x)$ as follows: Let $\Omega(n)$ denote the set of indices $i$ for which the center of the ball $B_{i}$ lies in $B(x, n)-B(x, n-1)$. (Take $B(x, 0) \equiv \varnothing$.) Then,

$$
|u(x)| \leq \sum_{i}\left|u_{i}(x)\right|=\sum_{n \geq 1} \sum_{i \in \Omega(n)}\left|u_{i}(x)\right|
$$

where the sum over $n$ on the right in (3.41) is a sum over positive integers. Note that the sum over integers $n$ is a finite sum but that the maximum value of $n$ allowed grows with $r$ as $\kappa \cdot r^{1 / 2}$. (The constant $\kappa$ is determined by the Riemannian metric on $X$.)

The second sum in (3.41) is bounded by

$$
\frac{z_{7} \cdot(N(n)-N(n-1))}{n^{3}}
$$

Thus,

$$
|u(x)| \leq z_{8} \cdot \sum_{n} \frac{(N(n)-N(n-1))}{n^{3}}=z_{8} \cdot \sum_{n} N(n) \cdot\left(\frac{1}{n^{3}}-\frac{1}{(n+1)^{3}}\right) .
$$

The last equality comes by rearranging terms. And, since one can bound the differences in the sum on the right by $z_{9} \cdot n^{-4}$, one has

$$
|u(x)| \leq z_{10} \cdot \sum_{n} \frac{N(n)}{n^{4}} .
$$

This last sum is uniformly bounded, independent courtesy of Lemma 3.8. Indeed, the right side of (3.44) is bounded by

$$
z_{11} \cdot \sum_{n=1}^{\infty} \frac{1}{n^{2}}<z .
$$




\section{The local structure of $\alpha^{-1}(0)$}

Fix a Spin ${ }^{\mathbb{C}}$ structure on $X$ with $S_{+}$given by (1.14) for some complex line bundle $E \rightarrow X$. The purpose of this section is to study the local structure of $\alpha^{-1}(0)$ for a large $r$ solution $(A,(\alpha, \beta))$ of (1.20). To put this task in context, remember that the principal goal of this article is to show that $\alpha^{-1}(0)$ is close to a (possibly singular) pseudo-holomorphic submanifold. Since the question of pseudo-holomorphicity is a local one, the issue of the local structure of the zero set of $\alpha$ naturally arises. The local structure of $\alpha^{-1}(0)$ is analyzed by comparing the fields $(A,(\alpha, \beta))$ near the given point with model fields which solve an $\mathbb{R}^{4}$ version of (1.20). (The first subsection below discusses the model problem for $\mathbb{R}^{4}$.) Proposition 4.2 summarizes the results of this section. Subsections $4 \mathrm{a}-\mathrm{c}$ are concerned directly with the local structure of $\alpha^{-1}(0)$.

Subsection 4d contains an application where it is proved that any of $\left\{1-|\alpha|^{2}\right.$, $\left.\beta, \nabla_{a} \alpha, \nabla_{A}^{\prime} \beta, F_{a}\right\}$ decays exponentially fast away from $\alpha^{-1}(0)$ with rate proportional to $r^{1 / 2}$.

The final subsection contains proofs of assertions in Proposition 4.1 about the solutions to the Seiberg-Witten equations on $\mathbb{R}^{4}$.

(a) The local model on $\mathbb{C}^{2}$. To begin, consider $\mathbb{R}^{4}$ with coordinates $\left(y^{1}, \ldots, y^{4}\right)$, Euclidean metric $\sum_{i} d y^{i} \otimes d y^{i}$, and standard symplectic form

$$
\omega_{0} \equiv d y^{1} \wedge d y^{2}+d y^{3} \wedge d y^{4} .
$$

The Euclidean metric identifies $\omega_{0}$ as a skew-symmetric endomorphism of $\mathbb{R}^{4}$ which defines a standard complex structure; the latter giving a standard $\mathbb{R}$ linear identification $\mathbb{R}^{4} \approx \mathbb{C}^{2}$. This complex structure will be implicit in much of the discussion below when the emphasis changes from the real or symplectic properties of $\mathbb{R}^{4}$ to its properties as the complex manifold $\mathbb{C}^{2}$.

The discussion below considers solutions $\left(A_{0},\left(\alpha_{0}, 0\right)\right)$ to $(1.20)$ as written on $\mathbb{R}^{4}$ with $r=1$ and with the symplectic form $\omega_{0}$ given in (4.1): Note that when $\mathbb{R}^{4}$ is thought of as $\mathbb{C}^{2}$, then (1.20) can be interpreted as saying, in part:

(1) $F_{A_{0}}$ is of type 1-1; thus $A_{0}$ defines a complex structure on the (trivial) complex line bundle over $\mathbb{C}^{2}$.

(2) With this complex structure on the trivial line bundle understood, then $\alpha_{0}$ is a holomorphic section.

It is important to note that the $\left(A_{0}, \alpha_{0}\right) \equiv\left(A_{0},\left(\alpha_{0}, 0\right)\right)$ which arise below are constrained as follows:

(1) $\left|\alpha_{0}\right| \leq 1$.

(2) $\left|P_{-} F_{A_{0}}\right| \leq\left|P_{+} F_{A_{0}}\right|=\frac{1}{4 \sqrt{2}}\left(1-\left|\alpha_{0}\right|^{2}\right)$.

(3) $\left|\nabla_{A_{0}} \alpha_{0}\right| \leq z \cdot\left(1-\left|\alpha_{0}\right|^{2}\right)$.

(4) For each $N \geq 1$, the integral of $\left(1-\left|\alpha_{0}\right|^{2}\right)$ over the ball of radius $N$ is bounded by $z \cdot N^{2}$.

(5) The integral over $\mathbb{R}^{4}$ of $\left|P_{+} F_{A_{0}}\right|^{2}-\left|P_{-} F_{A_{0}}\right|^{2}$ is finite.

Here, $z$ is a constant. 
Note for future references that the fields $\left(A_{0}, \alpha_{0}\right)$ which obey (4.3) have the following properties:

Proposition 4.1. Let $\left(A_{0},\left(\alpha_{0}, 0\right)\right)$ be a solution to $(1.20)$ on $\mathbb{R}^{4}$ using $r=1$ and the symplectic form $\omega_{0}$. Suppose that (4.3) is obeyed. Then

(1) Either $\left|\alpha_{0}\right|<1$ everywhere, or else $\left|\alpha_{0}\right| \equiv 1$ and $\left(A_{0}, \alpha_{0}\right)$ is gauge equivalent to the trivial solution $(0,1)$. In the former case, $\alpha_{0}^{-1}(0) \neq \varnothing$ and $\alpha_{0}^{-1}(0)$ is the zero set of a polynomial in the complex coordinates for $\mathbb{R}^{4}=\mathbb{C}^{2}$.

(2) Either $\left|P_{-} F_{A_{0}}\right|<\left|P_{+} F_{A_{0}}\right|$ everywhere, or else $\left|P_{-} F_{A_{0}}\right| \equiv\left|P_{+} F_{A_{0}}\right|$ everywhere and there is a $\mathbb{C}$ linear map $s: \mathbb{C}^{2} \rightarrow \mathbb{C}$ which is such that $\left(A_{0}, \alpha_{0}\right)$ are gauge equivalent to the s-pull back of a pair $\left(A_{1}, \alpha_{1}\right)$ of connection $A_{1}$ on the trivial line bundle over $\mathbb{C}$ and holomorphic section $\alpha_{1}$ of the said bundle. To be precise, $\left(A_{1}, \alpha_{1}\right)$ on $\mathbb{C}$ obey the vortex equation

$$
\begin{gathered}
i F_{A_{1}}=\frac{1}{4}\left(1-\left|\alpha_{1}\right|^{2}\right) \cdot \omega_{1}, \\
\bar{\partial}_{A_{1}} \alpha_{1}=0
\end{gathered}
$$

and are such that $i F_{A_{1}}$ is integrable over $\mathbb{R}^{2}$. In (4.4), $\omega_{1}$ is the Euclidean volume element on $\mathbb{R}^{2}$.

(3) Solutions $\left(A_{1}, \alpha_{1}\right)$ on $\mathbb{R}^{2}$ to the vortex equation in (4.4) having integrable $i F_{A_{1}}$ have the following properties:

(i) The integral of $i F_{A_{1}}$ over $\mathbb{R}^{2}$ equals $2 \pi n$ for $n \in\{0,1, \ldots\}$.

(ii) This integer $n$ (the vortex number) counts the number of zeros with multiplicities of $\alpha_{1}$.

(iii) For any given finite set $\Lambda$ of pairs consisting of a point in $\mathbb{C}$ and a positive integer, there is a unique (up to gauge equivalence) solution $\left(A_{1}, \alpha_{1}\right)$ to (4.4) having $i F_{A_{1}}$ integrable and having $\alpha_{1}$ vanishing at each point of $\Lambda$ to the given integer order. (And, $\alpha_{1}$ vanishes at no other points.)

(4) The integral of $\left|P_{+} F_{A_{0}}\right|^{2}-\left|P_{-} F_{A_{0}}\right|^{2}$ over $\mathbb{R}^{4}$ is a (non-negative) integer multiple of $4 \pi^{2}$.

(5) Given the constant $z$ in (4.3)(4), there exists an upper bound to the order of vanishing of $\alpha_{0}$ at any point in $\mathbb{R}^{4}$.

(6) The set of gauge equivalence classes of $\left(A_{0}, \alpha_{0}\right)$ as above which obeys (4.3) for a fixed value of $z$ and for a fixed upper bound on the integral of $\left|P_{+} F_{A_{0}}\right|^{2}-\left|P_{-} F_{A_{0}}\right|^{2}$ over $\mathbb{R}^{4}$ is sequentially compact with respect to convergence on bounded subsets of $\mathbb{R}^{4}$ in the $C^{\infty}$ topology.

(7) Given the value of $z$ in $(4.3)(4)$, there exists $z_{1}>0$ such that

$$
\left(1-\left|\alpha_{0}\right|^{2}\right)+\left|\nabla_{A_{0}} \alpha_{0}\right|^{2} \leq z_{1} \cdot \exp \left(-\frac{1}{z_{1}} \cdot \operatorname{dist}\left(\cdot, \alpha_{0}^{-1}(0)\right)\right) \text {. }
$$

Except for assertion (4) (which is not used here), this proposition is proved in the last subsection, below. The proof of assertion (4) will appear elsewhere.

(b) From fields on $X$ to fields on $\mathbb{C}^{2}$. Gaussian coordinate systems make the linear space $\mathbb{R}^{4}$ (alias $\mathbb{C}^{2}$ ) relevant to the analysis on the manifold $X$. Indeed, consider a point $x \in X$. An orthonormal frame for the tangent bundle at $x$ defines a Gaussian coordinate system at $x$. Remember that such a coordinate system defines an embedding $h: \mathbb{R}^{4} \rightarrow X$ which sends the origin to $x$ and which pulls back the Riemannian metric $g$ to a metric which is equal to the Euclidean metric to 
second order at the origin. Note that the frame can be chosen so that the symplectic form on $X$ pulls back as

$$
h^{*} \omega=d y^{1} \wedge d y^{2}+d y^{2} \wedge d y^{4}+\mathcal{O}(|y|) .
$$

Now, let $E$ be a given complex line bundle over $X$ and use $E$ to define the Spin ${ }^{\mathbb{C}}$ structure whose $S_{+}$bundle is given in (1.14). Fix some $r \geq 1$, and fix attention on a solution $(A,(\alpha, \beta))$ to $(1.20)$ for the given $\operatorname{Spin}^{\mathbb{C}}$ structure. Pull-back by $h$ defines $(A,(\alpha, \beta))$ as fields on $\mathbb{R}^{4}$.

Given $\lambda>0$, define the dilation map $\delta_{\lambda}: \mathbb{R}^{4} \rightarrow \mathbb{R}^{4}$ by its action on the coordinate functions $y$ :

$$
\delta_{\lambda}^{*} y=\lambda^{-1} y .
$$

With $x \in X$ chosen, set $\lambda \equiv \sqrt{r}$ and define fields $(\underline{A},(\underline{\alpha}, \beta))$ on $\mathbb{R}^{4}$ by the rule

$$
(\underline{A},(\underline{\alpha}, \underline{\beta})) \equiv \delta_{\lambda}^{*} h^{*}(A,(\alpha, \beta)) .
$$

On the ball of radius $r^{-1 / 2}$, the fields $(\underline{A},(\underline{\alpha}, \underline{\beta}))$ as defined in (4.7) obey:

$$
\begin{aligned}
& \text { (1) }|\underline{\alpha}|(y)=|\alpha|\left(r^{-1 / 2} y\right) \leq \mathcal{O}(1) . \\
& \text { (2) }|\underline{\beta}|(y)=|\beta|\left(r^{-1 / 2} y\right) \leq \mathcal{O}\left(r^{-1 / 2}\right) . \\
& \text { (3) }\left|\nabla_{\underline{a}} \underline{\alpha}\right|(y)=r^{-1 / 2}\left|\nabla_{a} \alpha\right|\left(r^{-1 / 2} y\right) \leq \mathcal{O}(1) . \\
& \text { (4) }\left|\nabla_{\underline{A}}^{\prime} \beta\right|(y)=r^{-1 / 2}\left|\nabla_{A}^{\prime} \beta\right|\left(r^{-1 / 2} y\right) \leq \mathcal{O}\left(r^{-1 / 2}\right) . \\
& \text { (5) }\left|F_{\underline{A}}\right|(y)=r^{-1}\left|F_{A}\right|\left(r^{-1 / 2} y\right) \leq \mathcal{O}(1) .
\end{aligned}
$$

Here, the norms are taken with respect to the Euclidean metric, and the size estimates are taken from Propositions 2.1, 2.3, 2.4 and 2.8 (or 3.4). In addition to (4.8), note that $(\underline{A},(\underline{\alpha}, \underline{\beta}))$ satisfy the Seiberg-Witten equations $(1.20)$ on $\mathbb{R}^{4}$ with $r \equiv 1$ and with a metric, $g^{\prime}$, and symplectic form $\omega^{\prime}$ which, where $|y| \leq \rho_{0} \cdot r^{1 / 2}$, satisfy:

$$
\begin{aligned}
& \text { (1) }\left|g^{\prime}-g_{0}\right| \leq z \cdot r^{-1}|x|^{2} \text {. } \\
& \text { (2) }\left|\nabla g^{\prime}\right| \leq z \cdot r^{-1} \cdot|x| . \\
& \text { (3) }\left|\nabla \nabla g^{\prime}\right| \leq z \cdot r^{-1} . \\
& \text { (4) }\left|\omega^{\prime}-\omega_{0}\right| \leq z \cdot r^{-1 / 2}|x| . \\
& \text { (5) }\left|\nabla \omega^{\prime}\right| \leq z \cdot r^{-1 / 2} .
\end{aligned}
$$

Here, $z$ and $\rho_{0}$ are $r$-independent constants; $g_{0}$ is the Euclidean metric and $\nabla$ is $g_{0}$ 's Levi-Civita covariant derivative.

With the preceding understood, consider:

Proposition 4.2. Fix a complex line bundle $E \rightarrow X$ and introduce the $\mathrm{Spin}^{\mathbb{C}}$ structure of (1.14). Fix $\epsilon>0, \delta>0, R \geq 1$ and $k \geq 0$. Then, there exists $r_{0} \equiv r_{0}\left(c_{1}(E), \epsilon, \delta, R, k\right) \geq 1$ such that for all $r \geq r_{0}$ the following is true: Let $(A,(\alpha, \beta))$ be any solution to (1.20) for the $\operatorname{Spin}^{\mathbb{C}}$ structure in (1.14) and for the given value of $r$.

Part A: Fix $x \in X$ and use $\lambda=r^{1 / 2}$, and fix a Gaussian coordinate system centered at $x$ to construct the fields $(\underline{A},(\underline{\alpha}, \underline{\beta}))$ as in $(4.7)$. Then, there is a smooth solution $\left(A_{0},\left(\alpha_{0}, 0\right)\right)$ of the $r=1$ version of $(1.20)$ on $\mathbb{R}^{4}$ (using the Euclidean metric on $\mathbb{R}^{4}$ and the standard symplectic form $\omega_{0}$ ) with the following properties: 
(1) The fields $\left(A_{0},\left(\alpha_{0}, 0\right)\right)$ depend on the chosen point $x$.

(2) After a gauge transformation, the fields $(\underline{A},(\underline{\alpha}, \beta))$ and $\left(A_{0},\left(\alpha_{0}, 0\right)\right)$ have distance less than $\epsilon$ from each other in the $C^{k}$ norm on 1-forms and complex valued functions on the ball in $\mathbb{R}^{4}$ of radius $R$ with center at the origin.

(3) The fields $\left(A_{0},\left(\alpha_{0}, 0\right)\right)$ obey (4.3). The constant $z$ depends on the Riemannian metric of $X$ and $c_{1}(E)$, but not on $r$ nor $(A,(\alpha, \beta))$.

Part B: As $x$ varies through $X$, one can assume without loss of generality that

(4) $\left|\alpha_{0}\right|$ is not constant if $|\alpha|(x)<1-\delta$.

(5) There exists a constant $z$ which depends only on $c_{1}(E)$ and the Riemannian metric and which has the following significance: The set of points for which the integral over the ball of radius $R$ in $\mathbb{R}^{4}$ of the corresponding $\left|P_{+} F_{A_{0}}\right|^{2}-$ $\left|P_{-} F_{A_{0}}\right|^{2}$ is greater than $\delta$ has a cover by balls of radius $R \cdot r^{-1 / 2}$ with less than $z \cdot \delta^{-1}$ elements.

(c) Proof of Proposition 4.2. This subsection proves Proposition 4.2. The proof is divided into two parts.

Part 1. This part proves the following lemma:

Lemma 4.3. Fix a $\mathrm{Spin}^{\mathbb{C}}$ structure as in (1.14). Let $\left\{r_{n}\right\}_{n \geq 1}$ be an unbounded sequence of positive numbers and let $\left\{\left(A_{n},\left(\alpha_{n}, \beta_{n}\right)\right)\right\}$ be a corresponding sequence of solutions to (1.20) for the given $\operatorname{Spin}^{\mathbb{C}}$ structure. Let $\left\{x_{n}\right\}$ be any corresponding sequence of points in $X$, and let $\left\{\varphi_{n}\right\}$ be a corresponding sequence of Gaussian coordinate charts for the $x_{n}$ 's. For each $n$, use $\varphi_{n}$ to construct $\left(\underline{A}_{n},\left(\underline{\alpha}_{n}, \underline{\beta}_{n}\right)\right)$ on $\mathbb{R}^{4}$ as described above. Then, after a gauge transformation of each $\left(\underline{A}_{n},\left(\underline{\alpha}_{n}, \underline{\beta}_{n}\right)\right)$ (which will not be noted explicitly) the resulting sequence $\left\{\left(\underline{A}_{n},\left(\underline{\alpha}_{n}, \underline{\beta}_{n}\right)\right)\right\}$ has an infinite subsequence which converges in the $C^{\infty}$ topology on compact subsets of $\mathbb{R}^{4}$ to a smooth solution $\left(A_{0},\left(\alpha_{0}, 0\right)\right)$ of the $r=1$ version of $(1.20)$ as defined by the Euclidean metric on $\mathbb{R}^{4}$ and the symplectic form $\omega_{0}$. Furthermore, this $\left(A_{0}, \alpha_{0}\right)$ satisfies (4.3).

Proof of Lemma 4.3. The convergence assertion follows from the fact that the equations in (1.20) are elliptic. (Thus, standard bootstrapping arguments (as in Chapter 6 of $[\mathrm{Mo}]$ ) can be applied given the initial a priori estimates in (4.8) and (4.9).) The fact that the limiting solution has the required form $\left(A_{0},\left(\alpha_{0}, 0\right)\right)$ follows from $(4.8)(2)$ and (4.8)(4). The constraints in (4.3)(1-3) follow directly from (4.8).

The fact that $\left(A_{0}, \alpha_{0}\right)$ satisfies (4.3)(4) follows from assertion (1) of Proposition 3.1. Indeed, if there is $\kappa>0$ and a ball of radius $N$ for which the integral of $\left(1-\left|\alpha_{0}\right|^{2}\right)$ is larger than $\kappa \cdot N^{2}$, then for all $n$ sufficiently large, the integral of $r_{n} \cdot\left(1-\left|\alpha_{n}\right|^{2}\right)^{2}$ over the ball of radius $\frac{N}{\sqrt{r}}$ with center $x_{n}$ is greater than $\frac{\kappa N^{2}}{2 r_{n}}$. Now invoke assertion (1) of Proposition 3.1 to bound $\kappa$.

In order to verify $(4.3)(5)$ for $\left(A_{0}, \alpha_{0}\right)$, suppose to the contrary that the integral in question is infinite (note that the integrand is non-negative due to the fact that (4.3)(2) has been shown to hold). Thus, given $\kappa>0$, there exists $N>1$ such that the integral of $\left|P_{+} F_{A_{0}}\right|^{2}-\left|P_{-} F_{A_{0}}\right|^{2}$ over the ball $B \subset \mathbb{R}^{4}$ with center at the origin and radius $N$ is at least $4 K$. Now, set $A \equiv A_{n}$ and $\underline{A} \equiv \underline{A}_{n}$. For all $n$ sufficiently large, the integral of $\left|P_{+} F_{\underline{A}}\right|^{2}-\left|P_{-} F_{\underline{A}}\right|^{2}$ over $B$ must be at least $4 K$. With small error, this last integral is equal to the integral of $\left|P_{-} F_{A}\right|^{2}-\left|P_{-} F_{A}\right|^{2}$ over the ball $B^{\prime} \subset X$ of radius $\frac{N}{\sqrt{r}}$ and center $x_{n}$. Hence, this integral must be at least $2 K$. 
On the other hand, the integral over $X$ of $\left|P_{+} F_{A}\right|^{2}-\left|P_{-} F_{A}\right|^{2}$ is equal to $c_{1}(E)$. $c_{1}(E)$, a fixed number. Thus, if $K$ is large, the integral over $X-B$ must be smaller than $-K$. Is this possible for large $K$ ? The answer is no, because Proposition 3.4 and (2.12) assert that

$$
\left|P_{+} F_{A}\right|^{2}-\left|P_{-} F_{A}\right|^{2} \geq-z \cdot r \cdot\left(1-|\alpha|^{2}\right)-z^{\prime},
$$

while Lemma 2.6 gives a uniform bound from below for the integral over $X$ of the right side of (4.10).

Part 2. With Lemma 4.3 understood, a proof of Proposition 4.2 requires the following four steps.

Step 1. Were Part A of the proposition false, there would exist an unbounded sequence $\left\{r_{n}\right\}$, with a corresponding sequence of solutions $\left\{\left(A_{n},\left(\alpha_{n}, \beta_{n}\right)\right)\right\}$ to (1.20); plus there would exist a sequence $\left\{x_{n}\right\}$ of points in $X$ with the following properties: For each index $n$, construct $\left(\underline{A}_{n},\left(\underline{\alpha}_{n}, \underline{\beta}_{n}\right)\right)$ from the solution $\left(A_{n},\left(\alpha_{n}, \beta_{n}\right)\right)$ and from $x_{n}$ and $\lambda=\sqrt{r}_{n}$ as instructed. Then, there is no data $\left(A_{0},\left(\alpha_{0}, 0\right)\right)$, as described in the proposition, which is $\epsilon$ close in the $C^{k}$ norm to any $\left(\underline{A}_{n},\left(\underline{\alpha}_{n}, \underline{\beta}_{n}\right)\right.$ ) (after some gauge transformation) on the standard radius $R$ ball in $\mathbb{R}^{4}$. It is this assertion that will be contradicted in the next step, thus proving Part A of Proposition 4.2.

Step 2. Note that Lemma 4.3 asserts that there is a subsequence of $\left(\underline{A}_{n},\left(\underline{\alpha}_{n}, \underline{\beta}_{n}\right)\right)$ which converges (after gauge transformations) in the $C^{\infty}$ topology on compact subsets of $\mathbb{R}^{4}$ to a solution, $\left(A_{0},\left(\alpha_{0}, 0\right)\right)$, to the $\mathbb{R}^{4}$ version of (1.20). And, this pair $\left(A_{0}, \alpha_{0}\right)$ obeys (4.3) with a constant $z$ which is determined solely by the Riemannian metric and $c_{1}(E)$. This contradicts the assumption in Step 1.

Step 3. This step proves assertion (4). Take $r$ large and take a solution $(A,(\alpha, \beta))$ to (1.20) for the given $\operatorname{Spin}^{\mathbb{C}}$ structure. Take a point $x$ where $|\alpha(x)|<1-\delta$. By Part A of Proposition 4.2, one concludes that for large $r$, the fields $(\underline{A},(\underline{\alpha}, \underline{\beta}))$ are, after a gauge transformation, very close on a very large ball to $\left(A_{0},\left(\alpha_{0}, 0\right)\right)$, a solution to $(1.20)$ on $\mathbb{R}^{4}$ (as defined with $r=1$ and the symplectic form $\omega_{0}$ ) which obeys (4.3). In particular, one can assume that $\left|\alpha_{0}(0)\right|<1-\frac{\delta}{2}$. With this understood, then (4.3)(4) implies that $\left|\alpha_{0}\right|$ is not constant.

Step 4. This step verifies assertion (5) of the proposition. Let $\Omega_{\delta} \subset X$ denote the set in question, and let $\mathcal{U}$ be a maximal (in number) cover of $\Omega_{\delta}$ by disjoint balls of radius $R \cdot r^{-1 / 2}$ with centers in $\Omega_{\delta}$. Let $N$ denote the number of elements in $\mathcal{U}$. The proof proceeds by bounding $N$ by some $z_{4} \cdot \delta^{-1}$. This is sufficient for the following reason: Because $\mathcal{U}$ is maximal, the balls which are concentric to those in $\mathcal{U}$ but with center $2 \cdot R$ must cover $\Omega_{\delta}$. Thus, if there are $N$ balls in $\mathcal{U}$, then $\Omega_{\delta}$ can be covered by less than $32 N$ balls.

To bound $N$, first digress to consider a point $x$ which is the center of a ball in $\mathcal{U}$. Since the integral over the radius $R$ ball with center 0 in $\mathbb{R}^{4}$ of $\left|P_{+} F_{A_{0}}\right|^{2}-$ $\left|P_{-} F_{A_{0}}\right|^{2}$ is at least $\delta$, the integral over the ball with center 0 and radius $R$ in $\mathbb{R}^{4}$ of $\left|P_{+} F_{\underline{A}}\right|^{2}-\left|P_{-} F_{\underline{A}}\right|^{2}$ will be at least $\delta-z_{1} \cdot \epsilon \cdot R^{4}$ for some universal constant $z_{1}$ which depends only on the constant in (4.3)(4). This follows from assertion (6) of Proposition 4.1 and assertion (2) of Proposition 4.2. Therefore, if $\delta>2 \cdot z_{1} \cdot \epsilon \cdot R^{4}$, the integral over this radius $R$ ball of $\left|P_{+} F_{\underline{A}}\right|^{2}-\left|P_{-} F_{\underline{A}}\right|^{2}$ will be at least $\delta / 2$. This implies that the integral of $\left|P_{+} F_{A}\right|^{2}-\left|P_{-} F_{A}\right|^{2}$ over the ball of radius $R \cdot r^{-1 / 2}$ in $X$ with center $x$ will be at least $\delta / 4$. 
With the digression understood, it follows that the balls from $\mathcal{U}$ contribute in total at least $N \cdot \delta / 4$ to the integral over $X$ of $\left|P_{-} F_{A}\right|^{2}-\left|P_{-} F_{A}\right|^{2}$. Now, remember that the latter integral is equal to $z_{2} \equiv 4 \pi^{2} c_{1}(E) \cdot c_{1}(E)$. Thus, the contribution to this integral from the complement of the union of the balls in $\mathcal{U}$ must be more negative than $z_{2}-N \cdot \delta / 4$. However, as indicated in (4.10), the contribution to this integral from this complement is bounded from below by some $-z_{3}$, where $z_{3}$ depends only on $c_{1}(E)$ and the Riemannian metric. Thus, $N$ is bounded by $z_{4} \cdot \delta^{-1}$.

(d) Exponential decay estimates. This subsection presents an application of Proposition 4.2 which asserts that everything of interest exponentially decays away from the set where $|\alpha|<\frac{1}{2}$. Here is the precise statement:

Proposition 4.4. Fix a complex line bundle $E \rightarrow X$; there is a constant $z$ which depends only on $c_{1}(E)$ and on the Riemannian metric and which has the following significance: Let $r \geq z$ and suppose that $(A,(\alpha, \beta))$ is a solution to $(1.20)$ for the Spin $^{\mathbb{C}}$ structure with $S_{+}$as in (1.14). Let $q \in\left\{r \cdot\left(1-|\alpha|^{2}\right), r^{3 / 2} \beta, F_{a}, r^{1 / 2} \nabla_{a} \alpha\right.$, $\left.r \nabla_{A}^{\prime} \beta\right\}$. Then

$$
|q|(x) \leq z \cdot r \cdot \exp \left(-\frac{1}{z} r^{1 / 2} \operatorname{dist}\left(x, \alpha^{-1}(0)\right)\right) .
$$

The remainder of this subsection is devoted to the

Proof of Proposition 4.4. The proof requires five steps. The strategy is to prove the assertion first for $\beta, \nabla_{a} \alpha$, and $\nabla_{A}^{\prime} \beta$ simultaneously. The assertions for $\left(1-|\alpha|^{2}\right)$ and $F_{a}$ are considered subsequently. Before starting, note that the usual convention about constants labeled as $z, z_{1}$, etc. will hold. That is, such constants depend only on $c_{1}(E)$ and the Riemannian metric.

Step 1. This step constitutes a digression of sorts to start the ball rolling with the following lemma:

Lemma 4.5. Let $E \rightarrow X$ be a complex line bundle. Given $\delta>0$, there exists a constant $z_{\delta}$ which depends on $\delta, c_{1}(E)$ and the Riemannian metric and which has the following significance: Let $r>z_{\delta}$ and let $(A,(\alpha, \beta))$ be a solution to (1.20) using the $\operatorname{Spin}^{\mathbb{C}}$ structure in (1.14). Let $x \in X$ be a point where $|\alpha|<1-\delta$. Then $\operatorname{dist}\left(x, \alpha^{-1}(0)\right)<z_{\delta} r^{-1 / 2}$.

Proof of Lemma 4.5. If $r$ is sufficiently large, assertion (4) of Proposition 4.2 gives a solution $\left(A_{0}, \alpha_{0}\right)$ to the $r=1$ version of $(1.20)$ on $\mathbb{R}^{4}$ for the point $x$ where $\left|\alpha_{0}\right|=1-\frac{\delta}{2}$. In this case, assertion (1) of Proposition 4.1 implies that $\alpha_{0}^{-1} \neq \varnothing$. Now invoke assertion (7) of Proposition 4.1 to learn that $\alpha_{0}$ vanishes at some point on $\mathbb{R}^{4}$ whose distance to the origin is less than $-z_{2} \ln (\delta)$. Here, $z_{2}$ depends only on $c_{1}(E)$ and on the metric because of assertion (3) of Proposition 4.2. This same assertion and assertion (5) of Proposition 4.1 bound the order of vanishing of $\alpha_{0}$ by a similar constant, $z_{3}$. Now fix $R>-2 \cdot z_{3} \ln (\delta)$ and take $k \geq 2 \cdot z_{3}$. With the preceding understood, Proposition 4.2 finds $z_{\delta}$ with the property that when $r>z_{\delta}$, then $\underline{\alpha}$ must also vanish on the radius $R$ ball in $\mathbb{R}^{4}$ with center 0 .

Step 2. This and the following two steps prove Proposition 4.4 for $\beta, \nabla_{a} \alpha$, and $\nabla_{A}^{\prime} \beta$. This step derives a differential inequality with an eye towards the eventual application of the comparison principle. The starting point is (2.38). Care with the 
triangle inequality replaces $(2.39)$ with

$$
\begin{aligned}
& \frac{1}{2} d^{*} d\left|\nabla_{a} \alpha\right|^{2}+\left|\nabla_{a} \nabla_{a} \alpha\right|^{2}+\frac{r}{4}|\alpha|^{2}\left|\nabla_{a} \alpha\right|^{2} \\
& \leq\left(z_{1} \cdot r\left(1-|\alpha|^{2}\right)+\frac{r}{16}\right)\left|\nabla_{a} \alpha\right|^{2} \\
&+z_{1} \cdot r|\beta|^{2}+z_{1} \cdot\left|\nabla_{A}^{\prime} \beta\right|^{2}+\frac{32}{r}\left|\nabla_{A}^{\prime} \nabla_{A}^{\prime} \beta\right|^{2} .
\end{aligned}
$$

(It is assumed here and below that $r$ is larger than some constant $z_{2}$ which depends only on $c_{1}(E)$ and on the Riemannian metric.)

Likewise, care with triangle inequalities replaces (2.41) with

$$
\begin{aligned}
& \frac{1}{2} d^{*} d\left|\nabla_{A}^{\prime} \beta\right|^{2}+\left|\nabla_{A}^{\prime} \nabla_{A}^{\prime} \beta\right|^{2}+\frac{r}{4}|\alpha|^{2}\left|\nabla_{A}^{\prime} \beta\right|^{2} \\
& \leq\left(z_{3} \cdot r \cdot\left(1-|\alpha|^{2}\right)+\frac{r}{16}\right)\left|\nabla_{a}^{\prime} \beta\right|^{2}+z_{3}|\beta|^{2} \\
&+z_{3} \cdot\left|\nabla_{a} \alpha\right|^{2}+\frac{32}{r}\left|\nabla_{a} \nabla_{a} \alpha\right|^{2} .
\end{aligned}
$$

Add (4.12) to $\frac{r}{32}$ times (4.13). The result (after a straightforward rearrangement, and after the discarding of certain manifestly positive terms from the left side) is the following inequality for $y \equiv\left(\left|\nabla_{a} \alpha\right|^{2}+\frac{r}{32}\left|\nabla_{A}^{\prime} \beta\right|^{2}\right)$ :

$$
\frac{1}{2} d^{*} d y+\frac{r}{4}|\alpha|^{2} y \leq\left(z_{4} \cdot r \cdot\left(1-|\alpha|^{2}\right)+\frac{r}{16}\right) \cdot y+z_{4} \cdot r \cdot|\beta|^{2} .
$$

Now take the constant $z_{3}$ from (2.7) and add $\frac{r^{2}}{16 z_{3}}$ times (2.7) to (4.14). Let

$$
y_{1} \equiv\left(\left|\nabla_{a} \alpha\right|^{2}+\frac{r}{32}\left|\nabla_{A}^{\prime} \beta\right|^{2}+\frac{r^{2}}{16 z_{3}}|\beta|^{2}\right) .
$$

This function obeys the differential inequality

$$
\frac{1}{2} d^{*} d y_{1}+\frac{r}{4}|\alpha|^{2} y_{1} \leq\left(z_{4} \cdot r \cdot\left(1-|\alpha|^{2}\right)+\frac{r}{8}\right) \cdot y_{1} .
$$

Here, $z_{5}$ is a constant which depends solely on $c_{1}(E)$ and on the Riemannian metric.

It follows from (4.15) and Lemma 4.5 that there exists a constant $z_{5}$ with the property that

$$
\frac{1}{2} d^{*} d y_{1}+\frac{r}{32} y_{1} \leq 0
$$

at all points $x \in X$ with $\operatorname{dist}\left(x, \alpha^{-1}(0)\right) \geq z_{5} \cdot r^{-1 / 2}$.

Step 3. Exponential decay estimates for $y_{1}$ follow from (4.16) using the comparison principle and a suitable choice of comparison function. Thus, the introduction of a suitable comparison function is the next order of business.

To begin, take $c \geq 1$ and $y \in X$. Then, consider the function

$$
H_{y}(\cdot) \equiv \frac{1}{\operatorname{dist}(\cdot, y)^{2}} \exp \left(-\frac{1}{c} r^{1 / 2} \cdot \operatorname{dist}(\cdot, y)\right)+c \cdot \exp \left(-\frac{1}{c} r^{1 / 2}\right) .
$$

The important feature of $H$ is that there is a choice for $c \geq 1$ which depends only on the Riemannian metric (and so is independent of $r$ ) and is such that

$$
\frac{1}{2} d^{*} d H+\frac{r}{32} H \geq 0
$$


when $\operatorname{dist}(x, y) \geq c \cdot r^{-1 / 2}$. (The proof of existence for such a constant $c$ is left as an exercise. But, here is a hint: First compute (4.18) in Gaussian coordinates about $y$.)

With $H_{y}$ understood, use Lemma 3.6 to find a maximal set $\left\{x_{i}\right\} \subset \alpha^{-1}(0)$ for which the balls $\left\{B_{i}^{\prime}\right\}$ with centers $\left\{x_{i}\right\}$ and radius $r^{-1 / 2}$ are disjoint. Then set

$$
h \equiv \sum_{i} H_{x i} .
$$

Up to a multiple, this $h$ is the desired comparison function. The following lemma describes its salient features:

Lemma 4.6. There is a constant $z_{6}$ which depends only on $c_{1}(E)$ and the Riemannian metric and which has the following significance:

(1) $\frac{1}{z_{6} \operatorname{dist}\left(x, \alpha^{-1}(0)\right)^{2}} \exp \left(-\frac{1}{c} r^{1 / 2} \cdot \operatorname{dist}\left(x, \alpha^{-1}(0)\right)\right) \leq h(x)$,

(2) $h(x) \leq z_{6} \cdot r \cdot \exp \left(-\frac{1}{c} r^{1 / 2} \cdot \operatorname{dist}\left(x, \alpha^{-1}(0)\right)\right)$, where $\operatorname{dist}\left(x, \alpha^{-1}(0)\right) \geq r^{-1 / 2}$.

Proof of Lemma 4.6. The lower bound follows from the definition. The upper bound is derived as follows: Given $x$, first find a point $x^{\prime} \in\left\{x_{i}\right\}$ whose distance to $x$ is minimal. Then, rearrange the sum in (3.18) as a sum indexed by integers $n=0,1, \ldots$ of a preliminary sum over the subset of points in $\left\{x_{i}\right\}$ with distance from $x^{\prime}$ in $\left[n \cdot r^{-1 / 2},(n+1) \cdot r^{-1 / 2}\right)$. Estimate the first sum by introducing the number $N(n)$ which is the number of points from $x_{i}$ which can lie in the ball of radius $n \cdot r^{-1 / 2}$. With the preceding understood, it follows that

$$
\begin{aligned}
h(x) \leq & \sum_{n} \frac{1}{d^{2}+n^{2} r^{-1}} \exp \left(-\frac{1}{c} r^{1 / 2} \cdot\left(d^{2}+n^{2} r^{-1}\right)^{1 / 2}\right)(N(n+1)-N(n)) \\
& +z_{7} \cdot r \cdot \exp \left(-\frac{1}{c} r^{1 / 2}\right) .
\end{aligned}
$$

Here, $d \equiv \operatorname{dist}\left(x, x^{\prime}\right)$. (The last term in (4.20) is the number of terms in (4.18) times the last term in (4.17). The former is bounded, courtesy of Proposition 3.1, by $z \cdot r$ ). Meanwhile, the sum in (4.20) can be estimated by using the rearrangement trick that was introduced in (3.43) and then invoking Proposition 3.1 to bound $N(n)$ by $z \cdot n^{2}$.

Step 4. Now, (4.16) and (4.18) plus Propositions 2.3 and 2.8 and Lemma 4.6 find a constant $z_{8}$ such that

(1) $\frac{1}{2} d^{*} d\left(y_{1}-z_{8} \cdot h\right)+\frac{r}{32}\left(y_{1}-z_{8} \cdot h\right) \leq 0 \quad$ where $\operatorname{dist}\left(x, \alpha^{-1}(0)\right) \geq z_{5} \cdot r^{-1 / 2}$.

(2) $y_{1} \leq z_{8} \cdot h \quad$ where $\operatorname{dist}\left(x, \alpha^{-1}(0)\right)=z_{5} \cdot r^{-1 / 2}$.

Thus, the maximum principle applies to bound $y_{1}$. This gives Proposition 4.4's asserted bounds for $\beta, \nabla_{a} \alpha$, and $\nabla_{A}^{\prime} \beta$.

Step 5 . This step uses the bounds for $\beta, \nabla_{a} \alpha$, and $\nabla_{A^{\prime}} \beta$ to derive the required bounds for $1-|\alpha|^{2}$ and for $F_{a}$. The arguments for both are similar, so only the case for $1-|\alpha|^{2}$ will be presented. To begin, note that (2.3) implies a differential 
inequality for $y \equiv|1-| \alpha^{2}||$ at points $x$ where $|\alpha|>1 / 4$ :

$$
\frac{1}{2} d^{*} d y+\frac{r}{32} y \leq z_{9} \cdot r \cdot \exp \left(-\frac{1}{2 c} r^{1 / 2} \operatorname{dist}\left(x, \alpha^{-1}(0)\right)\right) .
$$

Now, let $h^{\prime}$ denote the function in (4.19) but as defined with $\frac{r}{2}$ instead of $r$. This function obeys the differential inequality

$$
\frac{1}{2} d^{*} d h^{\prime}+\frac{r}{32} h^{\prime} \geq \frac{r}{64} h^{\prime}
$$

where $\operatorname{dist}\left(x, \alpha^{-1}(0)\right) \geq 2 r^{-1 / 2}$.

Now use (4.22) and (4.23) plus Lemma 4.6 to find a constant $z_{10}$ (which depends only on $c_{1}(E)$ and the metric) with the following significance: Introduce $y_{1} \equiv$ $y-\frac{z_{10}}{r} h^{\prime}$. This function obeys

$$
\frac{1}{2} d^{*} d y_{1}+\frac{r}{32} y_{1} \leq 0 \quad \text { where } \operatorname{dist}\left(x, \alpha^{-1}(0)\right) \geq z_{10} \cdot r^{-1 / 2},
$$

and it is non-positive where $\operatorname{dist}\left(x, \alpha^{-1}(0)\right)=z_{10} \cdot r^{-1 / 2}$. With this understood, the maximum principle gives Proposition 4.4's claim for $\left.|1-| \alpha\right|^{2} \mid$.

(e) Proofs for $\mathbb{C}^{2}$. The purpose of this subsection is to prove the assertions (1)(3) and (5)-(7) of Proposition 4.1. The fourth assertion of Proposition 4.1 will be proved elsewhere.

It is convenient to prove the assertions in a different order than presented.

Proof of assertion (6). The equations in question are elliptic in a ball with the gauge fixing condition $d * A_{0}=0$ in said ball. Already, $\alpha_{0}$ and $F_{A_{0}}$ are pointwise bounded in (4.3)(3). Thus, standard elliptic bootstrapping arguments apply (as in Chapter 6 of $[\mathrm{Mo}]$ ) to give a priori estimates of derivatives to any order of the fields $\left(A_{0}, \alpha_{0}\right)$. This implies that any sequence of $\left(A_{0}, \alpha_{0}\right)$ 's has a subsequence which converges on compact subsets of $\mathbb{R}^{4}$. Thus, (4.3)(1-4) all hold for the limiting pair $\left(A_{0}, \alpha_{0}\right)$. Since $\left|P_{+} F_{A_{0}}\right|^{2}-\left|P_{-} F_{A_{0}}\right|^{2}$ is a nowhere negative function, the integral of this function for the limiting fields $\left(A_{0}, \alpha_{0}\right)$ is no greater than the limit of the integrals of this function for the elements in the subsequence.

Proof of assertion (2). The proof of this assertion requires four steps.

Step 1. To prove this assertion it is necessary first to prove the part of assertion (1) which claims that $\left|\alpha_{0}\right|<1$ everywhere or else $\left|\alpha_{0}\right| \equiv 1$. This claim follows using the strong maximum principle and the $\mathbb{R}^{4}=\mathbb{C}^{2}$ version of $(2.3)$ with $\beta \equiv 0$ and $r \equiv 1$. Note that if $\left|\alpha_{0}\right| \equiv 1$, then $F_{A_{0}} \equiv 0$ and $\left(A_{0}, \alpha_{0}\right)$ is gauge equivalent to the trivial solution $(0,1)$.

Step 2. Now turn to the proof proper of assertion (2). The $\mathbb{C}^{2}$ version of (2.19) (with $r=1$ and with $\mathbb{R}$ and $\beta$ both zero) plus the strong maximum principle assert that the inequality in (4.3)(2) is an equality everywhere if it holds at a single point.

With the preceding understood, assume that (4.3)(2) holds everywhere. This is equivalent to asserting that

$$
F_{A_{0}} \wedge F_{A_{0}} \equiv 0
$$

everywhere. Think of $\mathbb{C}^{2}$ as $\mathbb{R}^{4}$ for a moment, and then one can conclude from (4.25) that $i F_{A_{0}}$, as a skew symmetric endomorphism of $T \mathbb{R}^{4}$, has a kernel everywhere. And, being skew symmetric, this kernel is everywhere at least 2-dimensional. Note that if $F_{A_{0}}=0$ at a point, then it follows from Step 1 that $F_{A_{0}} \equiv 0$ and the solution 
is gauge equivalent to the trivial solution $(0,1)$. Since this solution is described by assertion (2), it can be assumed without loss of generality that $F_{A_{0}}$ is nowhere vanishing. Thus, as $x$ moves over $\mathbb{C}^{2}=\mathbb{R}^{4}$, the kernel of $i F_{A_{0}}$ defines a dimension 2 distribution $\mathcal{D} \subset T \mathbb{R}^{4}$.

Lemma 4.7. The distribution $\mathcal{D} \subset T \mathbb{R}^{4}$ is integrable. And, it is also complex in that it is mapped to itself by the complex structure which defines $\mathbb{R}^{4}$ as $\mathbb{C}^{2}$.

Proof of Lemma 4.7. The integrability of $\mathcal{D}$ follows from the fact that $F_{A_{0}}$ is a closed form. The fact that $\mathcal{D}$ is complex is an algebraic consequence of the fact that $F_{A_{0}}$ is a form of type 1-1 on $\mathbb{C}^{2}$. (This last property of $F_{A_{0}}$ was alluded to in $(4.2)$.)

This lemma implies that the integral manifolds of $\mathcal{D}$ define a foliation of $\mathbb{C}^{2}$ with each leaf being a complex submanifold.

Step 3. This step proves that the foliation from Step 2 is one of the standard foliations by complex dimension 1 subvector spaces. The argument to prove this is as follows: The distribution $\mathcal{D}$ is defined as the kernel of $i F_{A_{0}}$ as a skew endomorphism of the tangent bundle. The kernel of the latter is the same as the kernel of $f \equiv \frac{i F_{A_{0}}}{\mid F_{A_{0}}}$. (Note that $\left|F_{A_{0}}\right|$ is nowhere vanishing, so $f$ is a smooth 2 -form on $\mathbb{C}^{2}$.) The claim now is that $f$ is a constant 2 -form. Of course, this claim establishes that the foliation from $\mathcal{D}$ is a foliation of $\mathbb{C}^{2}$ by linear $\mathbb{C}$ 's.

With the preceding understood, use $f_{ \pm}$to denote $\sqrt{2} \cdot P_{ \pm} f$ and remark that $f_{+}$ is a constant multiple of $\omega_{0}$ by inspection. (Needless to say, $\omega_{0}$ is constant.) As for $f_{-}$, consider analog of (2.18) on $\mathbb{C}^{2}$ with $\beta \equiv 0$ and $r=1$ :

$$
\frac{1}{2} \nabla^{*} \nabla \mu+\frac{1}{4}\left|\alpha_{0}\right|^{2} \mu=\frac{i}{4} P_{-}\left(\nabla_{A_{0}} \alpha_{0}^{*} \wedge \nabla_{A_{0}} \alpha_{0}\right) .
$$

Contracting both sides of this equation with $f_{-}$gives the equation

$$
\begin{gathered}
\frac{1}{2} d^{*} d\left|P_{-} F_{A_{0}}\right|+\left|P_{-} F_{A_{0}}\right| \cdot\left|\nabla f_{-}\right|^{2}+\frac{1}{4}\left|\alpha_{0}\right|^{2}\left|P_{-} F_{A_{0}}\right| \\
=\frac{i}{4}\left\langle f_{-}, \nabla_{A_{0}} \alpha_{0}^{*} \wedge \nabla_{A_{0}} \alpha_{0}\right\rangle .
\end{gathered}
$$

Here, $\langle$,$\rangle denotes the Euclidean inner product on \Lambda_{-}$. Meanwhile, there is a similar equation for the norm of $P_{+} F_{A_{0}}$ : this reads

$$
\frac{1}{2} d^{*} d\left|P_{+} F_{A_{0}}\right|+\frac{1}{4}\left|\alpha_{0}\right|^{2}\left|P_{+} F_{A_{0}}\right|=\frac{1}{4 \sqrt{2}}\left|\nabla_{A_{0}} \alpha_{0}\right|^{2} .
$$

Since $\left|P_{+} F_{A_{0}}\right|$ and $\left|P_{-} F_{A_{0}}\right|$ are equal, subtracting (4.28) from (4.27) gives the identity

$$
\left|P_{-} F_{A_{0}}\right| \cdot\left|\nabla f_{-}\right|^{2}=\frac{1}{4 \sqrt{2}}\left(i \cdot\left\langle\sqrt{2} f_{-}, \nabla_{A_{0}} \alpha_{0}^{*} \wedge \nabla_{A_{0}} \alpha_{0}\right\rangle-\left|\nabla_{A_{0}} \alpha_{0}\right|^{2}\right) .
$$

The right side of (4.29) is non-positive, while the left side is non-negative. Thus, both sides must be zero. Here are the implications:

(1) $\frac{i F_{A_{0}}}{\left|F_{A_{0}}\right|}$ is constant.

(2) $\nabla_{A_{0}} \alpha_{0}^{*} \wedge \nabla_{A_{0}} \alpha_{0}$ is proportional to $i F_{A_{0}}$.

(To obtain $(4.30)(2)$, note that the vanishing of the right side of (4.29) implies that the anti-self-dual projection of $\nabla_{A_{0}} \alpha_{0}^{*} \wedge \nabla_{A_{0}} \alpha_{0}$ must be along $P_{-} F_{A_{0}}$. Its 
self-dual projection is a priori along $P_{+} F_{A_{0}}$ because $\alpha_{0}$ is $A_{0}$-holomorphic. Also, (4.29) implies that both of these projections have the same length.)

Step 4. As remarked, (4.30)(1) implies that $i F_{A_{0}}$ pulls back to zero on a standard foliation of $\mathbb{C}^{2}$ by complex lines, while (4.30)(2) implies that $\alpha_{0}$ is $A_{0}$-covariant along these same complex lines.

The preceding establishes all of assertion (2) except for the fact that $i F_{A_{1}}$ in (4.4) is integrable. This last point follows from (4.3)(4).

Proof of assertion (3). This is established in [T4].

Proof of assertion (5). If no such bound existed, there would be a sequence of $\left(A_{0}, \alpha_{0}\right)$ 's whose $\alpha_{0}$ vanished to higher and higher order at the origin. By invoking assertion (6) there would be a subsequence which converged in the $C^{\infty}$ topology on compact subsets to a solution which is described by (4.3). This solution would have its $\alpha_{0}$ vanishing to infinite order at the origin. And, its $\alpha_{0}$ could not be constant because of (4.3)(4). But this is impossible because $\alpha_{0}$ is holomorphic.

Proof of assertion (1). Step 1 of the proof of assertion (2) deals with the first sentence of assertion (1).

The fact that $\alpha_{0}^{-1}(0)$ is the zero set of a complex polynomial follows from [Rut] (see also [St]). But, to apply the results in [Rut], remark first that (4.3)(5) plus assertions (2), (5) and (6) plus (4.3)(4) imply that $\alpha_{0}^{-1}(0)$ has the following property: Fix $R \geq 1$ and consider a sequence of balls $\left\{B_{i}\right\} \subset \mathbb{C}^{2}$ of radius $R$ whose centers are on $\alpha_{0}^{-1}(0)$. Suppose that the sequence of centers is unbounded, with increasing distance from the origin. Then the sequence of subsets $\left\{\left.\left(A_{0}, \alpha_{0}\right)\right|_{B_{i}}\right\}$ converges (after translation to the origin and complex rotations of the coordinates) to some vortex solution, which means that $\alpha_{0}^{-1}(0) \cap B_{i}$ converges to the intersection of the standard radius $R$ ball with a complex plane through the origin. This plane should be counted with some a priori bounded multiplicity. Assertion (5) supplies the a priori bound. These last facts imply that for large enough $N$, the integral in (4.3)(4) bounds the area of $\alpha_{0}^{-1}(0)$ in the radius $N$ ball.

Finally, consider the assertion that $\alpha_{0}^{-1}(0) \neq \varnothing$ if $\left|\alpha_{0}\right|<1$. There are three steps to this proof.

Step 1. This step considers the possibility of local minima for solutions to (1.20) on $\mathbb{C}^{2}$.

Lemma 4.8. Let $\left(A_{0}, \alpha_{0}\right)$ be a smooth solution to the $r=1, \omega=\omega_{0}$ and $\beta \equiv 0$ version of (1.20) on $\mathbb{C}^{2}$. Then $\left|\alpha_{0}\right|$ has no non-zero local minima unless $\left|\alpha_{0}\right| \equiv 1$.

Proof of Lemma 4.8. Consider the $r=1, \omega=\omega_{0}$ and $\beta \equiv 0$ version of (2.3) on $\mathbb{C}^{2}$ at a hypothetical non-zero local minimum of $\left|\alpha_{0}\right|$ where $\left|\alpha_{0}\right|<1$. There are three terms on the left side. The first term is non-positive and the third term is negative. Thus, a contradiction ensues with the claim that the second term is zero. To prove this claim, remark that as $\alpha_{0} \neq 0$ near the minimum, one can write $\alpha_{0} \equiv\left|\alpha_{0}\right| \cdot u$ with $|u|=1$. Thus, at the critical point, the second term on the left side of (2.2) is equal to $\left|\alpha_{0}\right|^{2} \cdot\left|\nabla_{a_{0}} u\right|^{2}$. Now, the $(0,1)$ projection of $\nabla_{a_{0}} u$ vanishes at the critical point since $\alpha_{0}$ is $A_{0}$-holomorphic. Consider the $(1,0)$ projection of $\nabla_{a_{0}} u$. This is the complex conjugate of the $(0,1)$ projection of $\nabla_{a_{0}}\left(u^{*}\right)$. However, $u^{*}=u^{-1}$ since $u$ has norm 1 , so the latter is the $(0,1)$ projection of $-u^{-2} \nabla_{a_{0}} u$ which is zero. 
Step 2. This step constitutes a digression to explain an important feature of the vortex solutions:

Lemma 4.9. There exists a constant $z>0$ with the following significance: Fix a positive integer $n$, and $\delta>0$. Let $\left(A_{1}, \alpha_{1}\right)$ be a solution to (4.4) on $\mathbb{R}^{2}$ which has integral $i F_{A_{1}}$ and vortex number $n$. Then $\left|\alpha_{1}\right|>1-\delta$ at points with distance $\frac{z \cdot n}{\delta}$ or more from $\alpha_{1}^{-1}(0)$.

Proof of Lemma 4.9. Let $y \in \mathbb{R}^{2}$ be a point where $\left|\alpha_{1}\right| \leq 1-\delta$, and let $d$ denote the distance from $y$ to $\alpha_{1}^{-1}(0)$. It follows from Lemma 4.8 that there is a path from $y$ to $\alpha_{1}^{-1}(0)$ along which $\left|\alpha_{1}\right|$ is less than $1-\delta$. Now note that $\left|\nabla_{A_{1}} \alpha_{1}\right|$ is bounded by a constant $c$ (which is independent of $\left(A_{1}, \alpha_{1}\right)$ and $n$ ). See [JT, Theorem 8.1]. This implies that $\left|\alpha_{1}\right|<1-\frac{\delta}{2}$ on a set whose area is at least $\frac{d \cdot \delta}{4 c}$. Since the integral of $\left(1-\left|\alpha_{1}\right|^{2}\right)$ is uniformly bounded by a multiple of $n$, the lemma follows.

Step 3. Now, suppose that $\left(A_{0}, \alpha_{0}\right)$ obeys (4.3) and $\alpha_{0}$ is never zero. First of all, it follows from assertions (2), (5) and (6) plus (4.3)(4) that on any sequence of balls of fixed radii with centers tending to infinity, the fields $\left(A_{0}, \alpha_{0}\right)$ must converge (up to gauge transformation) to those of a vortex solution as described in assertions (2) and (3) of Proposition 4.1 with an a priori upper bound on the vortex number. With the assumption being that $\alpha_{0}$ does not vanish, Lemma 4.8 implies that these vortex solutions must all be trivial. Thus, $\left|\alpha_{0}\right|$ tends to 1 as $|x| \rightarrow \infty$ on $\mathbb{C}^{2}$.

Now it follows from Step 1 that $\left|\alpha_{0}\right|$ cannot have a non-zero local minimum unless $\left|\alpha_{0}\right| \equiv 1$.

Proof of assertion (7). The proof is identical in most respects to the proof of Proposition 4.4 , so only a sketch will be given. The main difference is that the analog of Lemma 4.5 must be proved without invoking assertion (7)! The $\mathbb{C}^{2}$ version of Lemma 4.5 is proved as follows: As remarked earlier, assertions (2), (5) and (6) of Proposition 4.1 plus (4.3)(4) imply that on any sequence of fixed radii balls with centers tending to infinity, the fields $\left(A_{0}, \alpha_{0}\right)$ converge (up to gauge equivalence) to a vortex solution with an a priori upper bound on the vortex number. This fact plus Lemma 4.9 imply Lemma 4.5 for the case $\mathbb{C}^{2}$.

The only other significant change in the argument for Proposition 4.4 is that the function $H_{y}$ in (4.17) should be modified by dropping the right most term.

With these changes understood, the remainder of the argument for assertion (7) continues almost word for word as that for Proposition 4.4; thus the details are left to the reader.

\section{Convergence to a Current}

The purpose of this section and the next is to prove Theorem 1.3 from Section 1. As remarked at the end of Section 1, the strategy is to take a sequence $\left\{r_{n}\right\}$ of $r$ values for Theorem 1.3 with the corresponding sequence $\left(A_{n},\left(\alpha_{n}, \beta_{n}\right)\right)$ of solutions to (1.20). The sequence $\left\{\frac{i}{2 \pi} F_{A_{n}}\right\}$ will then be regarded as a sequence of currents $\mathcal{F}_{n}$, where

$$
\mathcal{F}_{n}(\mu) \equiv \int \frac{i}{2 \pi} F_{A_{n}} \wedge \mu
$$

This sequence will be shown to have a subsequence which converges to a current, $\mathcal{F}$, whose support is a closed set $C$ with finite 2-dimensional Hausdorff measure. The current $\mathcal{F}$ will then be used to define an integer valued, homotopy invariant (called 
$I$ ) of a certain class of maps from 2-dimensional disks into $X$. A crucial point is that this homotopy invariant assigns a positive integer to any symplectically embedded disk with non-trivial intersection, but compactly supported intersection with $C$. This crucial result is stated in Proposition 5.6, below. The proof of Proposition 5.6 ends this section.

(a) Alternate approaches. There is a second route to proving Theorem 1.3 which will not be pursued here: Using the local picture in Proposition 4.2, a straightforward (though tedious) construction produces a symplectic immersion of a curve near each $\alpha_{n}^{-1}(0)$. (Each point in $\alpha_{n}^{-1}(0)$ has its associated data $\left(A_{0}, \alpha_{0}\right)$ from Proposition 4.2. The set $\alpha_{0}^{-1}(0)$ can be scaled down (by the inverse of (4.6)) and identified as a symplectic subvariety in $X$. Then as $x$ varies through $\alpha_{n}^{-1}(0)$, the latter are pieced together to give the symplectic immersion. In this regard, here is a corollary of Proposition 4.2: If $n$ is large and if, for all $x \in \alpha_{n}^{-1}(0)$, the data $\left(A_{0}, \alpha_{0}\right)$ is a vortex solution from $\mathbb{C}$ with vortex number 1 , then $\alpha_{n}^{-1}(0)$ will already be an embedded symplectic curve.)

In any event, these symplectic immersions become pointwise pseudo-holomorphic as $n$ tends to $\infty$ in the sense that the area form differs negligibly from the pullback of the symplectic form in the $n \rightarrow \infty$ limit. Thus, the area of the immersion approaches the theoretical minimum value $2 \pi[\omega] \cdot c_{1}(E)$ as $n \rightarrow \infty$. (Here, $[\omega]$ denotes the cohomology class of the symplectic form and · denotes the cup product pairing in cohomology.) The fundamental compactness theorem for bounded area, rectifiable currents in $[\mathrm{FF}]$ (see Theorem 3.3 in [La]) can then be quoted to find a limit which is an area minimizing, 2-dimensional rectifiable current. Then a hard theorem of Sheldon Chang [Ch] can be used to infer that the latter is the image of a surface by a map; and the area being $2 \pi[\omega] \cdot c_{1}(E)$ implies that the map is pseudo-holomorphic.

If rectifiable currents are not to your liking, there is a third route to proving Theorem 1.3. This takes the sequence of symplectic immersions previously alluded to and uses a generalization of Ye's proof (in [Ye]) of Gromov's compactness theorem (in $[\mathrm{Gr}]$ ) to prove that there is a limit which is the image of a complex curve by a pseudo-holomorphic map. (Note that a uniform Hölder estimate exists for a symplectic immersion on any disk where the harmonic map energy is small.) This third approach is complicated only because the aforementioned sequence of symplectic immersions need not admit (apparently) a bound on the genus of the immersing surface. However, with the help of the monotonicity formula in Proposition 3.1, the unpleasant growth of the genus with the sequence index $n$ can be dealt with.

(b) The sequence of currents. Define the sequence $\left\{\mathcal{F}_{n}\right\}$ of currents as in (5.1). Because of Lemma 2.6 and Proposition 3.4, this sequence is uniformly bounded with respect to the mass norm. That is,

$$
M\left(\mathcal{F}_{n}\right) \equiv \sup _{0 \neq \mu \in \Omega^{2}(X)}\left\{\left|\mathcal{F}_{n}(\mu)\right| \cdot\left(\sup _{x \in X}|\mu(x)|\right)^{-1}\right\}
$$

obeys

$$
M\left(\mathcal{F}_{n}\right) \leq \frac{1}{2 \pi}\left\|F_{A_{n}}\right\|_{L^{1}} \leq[\omega] \cdot c_{1}(E)+\frac{z_{1}}{r},
$$

where $z_{1}$ depends only on $c_{1}(E)$ and the Riemannian metric. (The convention henceforth in this section about constants which are labeled $z_{\text {anything }}$ is that they 
depend only on $c_{1}(E)$ and the Riemannian metric. In particular, they are independent of the index $n$.)

The bound in (5.3) implies that there is a subsequence of the original sequence of currents (henceforth relabeled as $\left\{\mathcal{F}_{n}\right\}$ ) which converges in the weak topology (as defined by the norm $M(\cdot)$ ) to a current, $\mathcal{F}$. Note that this current is closed (as each $\mathcal{F}_{n}$ is closed) in the sense that it annihilates the image of $d$; and $\mathcal{F}$ is integral in the sense that its value is an integer on any closed form which evaluates as an integer on all closed cycles. The current $\mathcal{F}$ is Poincaré dual to $c_{1}(E)$ in the sense that

$$
\mathcal{F}(\mu)=[\mu] \cdot c_{1}(E)
$$

for any closed 2-form $\mu$.

(c) The support of $\mathcal{F}$. The purpose of this subsection is to identify the support of the current $\mathcal{F}$. The monotonicity formula in Proposition 3.1 plays the key role throughout.

To begin, note that according to Lemma 3.6, there is a constant $z$ which depends only on $c_{1}(E)$ and the Riemannian metric and which has the following significance: Fix $r \geq 1$ and a solution $(A,(\alpha, \beta))$ to $(1.20)$ for the $\operatorname{Spin}^{\mathbb{C}}$ structure in (1.14). If $\rho \geq z \cdot r^{-1 / 2}$, then no set of disjoint balls of radius $\rho$ with centers on $\alpha^{-1}(0)$ has more than $z \cdot \rho^{-2}$ elements.

Let $r$ and $(A,(\alpha, \beta))$ be as in the preceding paragraph. Fix $\rho>z \cdot r^{-1 / 2}$ and let $\Lambda^{\prime}$ be a maximal (in number) set of disjoint balls of radius $\rho$ with centers on $\alpha^{-1}(0)$. Let $\Lambda$ be the set of balls of radius $2 \rho$ with the same centers as the balls in $\Lambda^{\prime}$. Because $\Lambda^{\prime}$ is maximal, the balls in $\Lambda$ provide a cover of $\alpha^{-1}(0)$.

With the preceding understood, for each positive integer $N$, and for each index $n$ for which $r_{n}>z^{2}(256)^{N}$, find a maximally disjoint set $\Lambda_{n}^{\prime}(N)$ of balls of radius $16^{-N}$ with centers in $\alpha_{n}^{-1}(0)$. Let $\Lambda_{n}(N)$ denote the corresponding set of concentric balls with radii $2 \cdot(16)^{-N}$. As remarked, the set $\Lambda_{n}(N)$ contains no more than $z \cdot(256)^{N}$ elements and its balls cover $\alpha_{n}^{-1}(0)$.

One may suppose that $z$ here is an integer, and with this understood, label and order the centers of the balls in $\Lambda_{n}(N)$, and then add the final point some number of times (if necessary) to make an ordered set $\left(x_{n}(1 ; N), \ldots, x_{n}\left(z(256)^{N} ; N\right)\right) \subset$ $\alpha_{n}^{-1}(0)$. Introduce $U_{n}(N)$ to denote the union of the set of balls with radii $4 \cdot(16)^{-N}$ and with centers at the $\left\{x_{n}(i ; N)\right\}$.

By a countable winnowing process, one can find an infinite subsequence of indices $n$ (hence labeled as $n=1,2, \ldots)$ for which the sequence of $\left\{x_{n}(i: N)\right\}_{n \geq 1}$ converges to some $x(i ; N) \in X$ for each pair $(i, N)$. With this understood, let $U(N)$ denote the union of the set of $z \cdot(256)^{N}$ balls with radii $r \cdot(16)^{-N}$ and with centers at the points $\{x(i ; N)\}$. It follows from the construction that the sets $\{U(N)\}_{N \geq 1}$ are nested in the sense that

Lemma 5.1. There is the inclusion $U(N+1) \subset U(N)$ as

$$
\operatorname{dist}(U(N+1), X-U(N)) \geq \frac{3}{2} 16^{-N} .
$$

Proof of Lemma 5.1. Note that $U_{n}(N+1) \subset U_{n}(N)$. Indeed, every point in the former has distance $\frac{1}{4} 16^{-N}$ or less from $\alpha_{n}^{-1}(0)$, and every point in $\alpha_{n}^{-1}(0)$ has distance $2 \cdot 16^{-N}$ or less from some $x_{n}(i ; N)$. Thus, every point in $U(N+1)$ has distance $2 \frac{1}{4} 16^{-N}$ or less from some $x_{n}(i ; N)$. Meanwhile, every point not in $U(N)$ has distance at least $4 \cdot 16^{-N}$ from all $x_{n}(i ; N)$. 
Given the nested set $\{U(N)\}$ above, define

$$
C \equiv \bigcap_{N} U(N)
$$

Lemma 5.2. The set $C$ has finite 2-dimensional Hausdorff measure. Furthermore, there is a constant $z_{1}>0$ which depends only on $c_{1}(E)$ and on the Riemannian metric and which has the following significance: Let $x \in C$ and let $B \subset X$ be a ball in $X$ of radius $\rho$ with $x$ as center. Then the Hausdorff measure of $C \cap B$ is at least $z_{1} \cdot \rho^{2}$. Finally, $C$ contains the support of $\mathcal{F}$.

Proof of Lemma 5.2. It follows from the construction that given any $\epsilon>0$, the set $C$ has a covering by no more than $z \cdot \epsilon^{-2}$ balls of radius $\epsilon$. Thus, $C$ has finite 2-dimensional Hausdorff measure, the constants from Lemma 3.6 giving the bound. The upper bound on the 2-dimensional measure of $C \cap B$ follows from Proposition 3.1 .

As for the assertion about the support of $\mathcal{F}$, consider a smooth form $\mu$ whose support is disjoint from $C$. Then the support will be disjoint from $U_{n}(N)$ for some $N$ and for all $n$ sufficiently large. Thus, according to Proposition 4.4,

$$
\left|\mathcal{F}_{n}(\mu)\right| \leq z \cdot r \cdot \exp \left(-z^{-1} r_{n}^{1 / 2} \cdot 4 \cdot 16^{-N}\right) \cdot \sup _{x}|\mu(x)| .
$$

It follows from (5.7) that $\lim _{n \rightarrow \infty}\left|\mathcal{F}_{n}(\mu)\right|=0$.

An additional corollary can be drawn from the preceding discussion, this being the "type" of $\mathcal{F}$. To explain, remember that the symplectic form and the metric together define an almost complex structure $J$ for $X$. This $J$ acts pointwise on $T^{*} X$ and so defines an involution $\mathfrak{J}$ on the $\Omega^{2}(X)$. Thus, there is dual involution (also called $\mathfrak{J}$ ) on currents. Say that a 2 -dimensional current is of type 1-1 if it is invariant under $\mathfrak{J}$.

Lemma 5.3. The current $\mathcal{F}$ is of type 1-1.

Proof of Lemma 5.3. Using a partition of unity for the cover of $X$ by $\{X-U(N+1)$, $U(N)\}$ decompose a 2-form $\mu$ into a sum $\mu_{1}+\mu_{2}$, where $\mu_{1}$ has support on $X-$ $U(N+1)$ and where $\mu_{2}$ has support on $U(N)$. According to (5.9), the value of $\mathcal{F}_{n}$ on $\mu_{1}$ tends to zero as $n$ tends to $\infty$ for fixed $N$. Meanwhile, the value of $\mathcal{F}_{n}$ on $\mu_{2}-\mathfrak{J} \mu_{2}$ involves only the non- $\mathfrak{J}$-invariant terms in $F_{A_{n}}$; namely the terms in $P_{+} F_{A_{n}}$ which are linear in $\beta_{n}$ and $\beta_{n}^{*}$ (see (1.20)). And, Hölder's inequality and Proposition 2.3 bound $\left|\mathcal{F}_{n}\left(\mu_{2}-\mathfrak{J} \mu_{2}\right)\right|$ by

$$
z \cdot \sup _{x} \mid \mu_{2} \cdot\left(\left\|r_{n}\left(1-\left|\alpha_{n}\right|^{2}\right)\right\|_{L^{1}} \cdot \operatorname{Volume}\left(U_{N}\right)+r_{n}^{-1}\right)^{1 / 2} .
$$

This last expression is $\mathcal{O}\left(16^{-N}\right)$ as $n \rightarrow \infty$, courtesy of Lemma 2.6 and the fact that the volume of $U_{n}(N)$ is no greater than $z \cdot 16^{-2 N}$. Since $N$ in (5.8) can be arbitrarily large, the lemma follows.

(d) Families of pseudo-holomorphic disks. As remarked previously, $C$ will be studied by analyzing its intersections with pseudo-holomorphic disks. The plan requires a varied family of such disks; and this subsection serves as a digression which describes the required family.

To begin, fix a point $x \in X$ and Gaussian normal coordinates centered at $x$ which identify a geodesic ball about $x$ with a ball in $\mathbb{R}^{4}$ and take $x$ to the origin. As in the previous chapter, identify $\mathbb{R}^{4}=\mathbb{C}^{2}$ so that $\left.\omega\right|_{x}=\omega_{0} \equiv d x^{1} \wedge d x^{2}+d x^{3} \wedge d x^{4}$. Use 
$\left(w_{0}, w_{1}\right)$ for the complex coordinates on $\mathbb{C}^{2}$. (Complex coordinates such as these will henceforth be called complex Gaussian coordinates centered at $x$.)

In these coordinates, the almost complex structure $J$ from $X$ agrees with the standard almost complex structure $J_{0}$ from $\mathbb{C}^{2}$ at the origin, but typically nowhere else. Thus, a $J_{0}$-holomorphic disk will not usually be $J$-holomorphic except at isolated points. With this understood, the plan for this subsection is to establish a perturbation lemma which constructs $J$-holomorphic disks from $J_{0}$-holomorphic ones. Of immediate interest in the next subsection are the $J$-pseudo-holomorphic versions of the family $\left\{D_{w}\right\}_{w \in \mathbb{C}}$ of holomorphic disks where

$$
D_{w} \equiv\{(\zeta, w):|\zeta|<\rho\} .
$$

A pseudo-holomorphic version of (5.9) is given in Lemma 5.4 below. Lemma 5.4 follows from a general perturbation result which is expressed as Lemma 5.5; various applications of Lemma 5.5 are sprinkled throughout subsequent subsections and Section 6 .

Lemma 5.4. There exists $\rho_{0}>0$ and $z$ which depend only on the symplectic form $\omega$ and the Riemannian metric and which have the following significance: Fix $x \in X$ and complex Gaussian coordinates $\left(w_{0}, w_{1}\right)$ centered at $x$ as above $\left(\right.$ so $\left.\left.\omega\right|_{x}=\omega_{0}\right)$. Suppose that $\rho<\rho_{0}$ and let $D \subset \mathbb{C}$ denote the disk of radius $\rho$. There is a diffeomorphism $\Theta: D \times D \rightarrow \mathbb{C}^{2}$ which obeys:

(1) For all $w \in D, \Theta\left(D_{w}\right)$ is a pseudo-holomorphic submanifold.

(2) For all $w \in D, \Theta\left(D_{w}\right)$ contains $(0, w)$.

(3) For all $w \in D$, $\operatorname{dist}((\zeta, w) ; \Theta(\zeta, w)) \leq z \cdot \rho \cdot|\zeta|$.

(4) For all $w \in D$, the derivatives of order $m$ of $\Theta$ are bounded by $z_{m} \cdot \rho$, where $z_{m}$ depends only on the Riemannian metric and on $\omega$.

This lemma is proved below. The proof requires a digression to consider the general set up for studying perturbations of a $J_{0}$-holomorphic disk. The perturbations in question are analogous to the holomorphic perturbation of $D_{w}$ as $\{(\zeta, w+f(\zeta))\}$, where $f=c_{m} \zeta^{m}+\cdots+c_{0}$ is a polynomial of degree $m$. In any event, the digression is summarized by Lemma 5.5. This lemma has two aspects, the first being an existence statement (which is used to prove Lemma 5.4) and the second a construction of a class of $J$-pseudo-holomorphic perturbations of a given $J$-pseudo-holomorphic disk.

To start the digression, introduce $D_{0} \equiv\{(\zeta, 0):|\zeta|<\rho\}$. Now, let $f: \mathbb{C} \rightarrow \mathbb{C}$ be a complex polynomial and let $u: \mathbb{C} \rightarrow \mathbb{C}$ be a smooth map. The task for this digression is to find $\rho_{0}$ and a smooth, complex valued function $\tau$ on $D_{0}$ so that the map $q: D_{0} \rightarrow \mathbb{C}^{2}$ which sends $\zeta$ to

$$
q(\zeta) \equiv(\zeta, u(\zeta)+f(\zeta)+\tau(\zeta))
$$

be $J$-pseudo-holomorphic when $\rho<\rho_{0}$.

The condition that $q$ be pseudo-holomorphic imposes a differential constraint on $\tau$ of the following schematic form:

$$
\frac{\partial \tau}{\partial \bar{\zeta}}+\left\{H_{0}+\frac{\partial u}{\partial \bar{\zeta}}\right\}+H_{1} \cdot d_{R} \theta+H_{4} \cdot\left[d \theta_{R} \otimes d \theta_{R}\right]=0
$$

Here, $\theta \equiv u+f+\tau$ and $d_{R} \theta$ is the differential of $\theta$ as a map from $\mathbb{R}^{2}$ to $\mathbb{R}^{2}$. In (5.11), each $H_{i}$ is the pull-back via $q$ of a smooth tensor, $\mathfrak{H}_{i}$, which is defined on a 
ball in $\mathbb{C}^{2}$ of some radius $\rho_{1}>0$ with center 0 ; on this ball, $\mathfrak{H}_{i}$ obeys

$$
\left|\nabla^{m} \mathfrak{H}_{i}\right|_{x} \leq z_{m} \cdot\left|\nabla^{m}\left(J-J_{0}\right)\right|_{x} .
$$

(Note that $\mathfrak{H}_{i}$ is constructed from the coefficients of $J-J_{0}$, a tensor on $\mathbb{C}^{2}$.)

It proves convenient to extend (5.12) as an equation over a somewhat larger disk in $\mathbb{C}$. For this purpose, choose a smooth function $\chi:[0, \infty) \rightarrow[0,1]$ which is 1 on $[0,1]$ and which vanishes on $[1.5, \infty)$. Let $\chi_{\rho}: \mathbb{C} \rightarrow[0,1]$ denote the function which sends $\zeta$ to $\chi\left(\rho^{-1} \cdot|\zeta|\right)$. Now write the right side of (5.11) as $Q$ and consider solving for a complex valued function $\tau$ on $\mathbb{C}$ which obeys

$$
\frac{\partial \tau}{\partial \bar{\zeta}}+\chi_{\rho} \cdot Q=0
$$

The plan is to search for a solution $\tau$ of the form

$$
\left.\tau\right|_{\zeta}=\left.c \cdot \int \frac{1}{(\zeta-\eta)}\left(\chi_{\rho} \cdot Q\right)\right|_{\eta} d^{2} \eta
$$

where $c$ is an appropriate constant. The search will cover the Banach space $\mathcal{K}_{\rho}$ of complex valued functions on $\mathbb{C}$ which have Hölder continuous second derivatives with exponent $1 / 2$ and which restrict to the radius $4 \rho$ circle (centered at 0 ) in the span of the functions $\left\{e^{-i \theta}, e^{-2 i \theta}, \ldots\right\}$. (The point is that the Green's function in (5.14) maps $C^{m, \delta}$ functions to $C^{m+1, \delta}$ with bounded norm for any integer $m \geq 0$ and $\delta \in(0,1)$. See, e.g. [DN].) It is convenient to introduce the following norm on this space:

$$
\begin{aligned}
\|\tau\| \equiv & \sup _{t \in \mathbb{C}}\left(|\tau|+\rho \cdot|d \tau|+\rho^{2} \cdot|\nabla d \tau|\right) \\
& +\rho^{5 / 2} \cdot \sup _{t, s \in \mathbb{C}} \frac{\left|\nabla d \tau_{t}-\nabla d \tau_{s}\right|}{|t-s|^{1 / 2}} .
\end{aligned}
$$

The details of this fixed point approach are straightforward and omitted save for the following lemma which summarizes:

Lemma 5.5. There exist constants $\rho_{0}>0$ and $z_{1}, z_{2}>0$ with the following significance: Suppose that $\rho<\frac{1}{2} \rho_{0}$ and suppose that $f=c_{m} \zeta^{m}+\cdots+c_{0}$ is a polynomial of degree $m$ with $\left\|\chi_{\rho} f\right\| \leq z_{1}$. Suppose that $u$ is a smooth, complex valued function on the radius $2 \rho$ disk with $\left\|\chi_{\rho} u\right\| \leq z_{1}$ also.

(1) Equation (5.17) has a unique solution $\tau$ in $\mathcal{K}_{\rho}$ with

$$
\|\tau\| \leq z_{2} \cdot\left(\rho^{2}+\left\|\chi_{\rho} u\right\|+\rho \cdot\left\|\chi_{\rho} f\right\|\right)
$$

(2) The function $\tau$ is smooth.

(3) The function $\tau$ is jointly infinitely differentiable with respect to $\zeta$ and to the variables $\left\{c_{k}\right\}$ of $f$; and $\left\|\frac{\partial \tau}{\partial c_{k}}\right\| \leq z_{k} \rho^{k+1}$.

Here, $z_{k}$ depends only on $k, \omega$ and the Riemannian metric.

(4) Suppose that $q_{0}: D \rightarrow D \times D$ which sends $\zeta$ to $(\zeta, u(\zeta))$ is J-pseudoholomorphic. Then $\|\tau\| \leq z_{2} \cdot \rho \cdot\left\|\chi_{\rho} f\right\|$ and $\left\|\frac{\partial \tau}{\partial c_{k}}\right\| \leq z_{k} \cdot \rho^{k+1}$.

(5) In any case the map $q$ in (5.10) is pseudo-holomorphic on D.

This lemma ends the digression.

Proof of Lemma 5.4. Lemma 5.4 follows from Lemma 5.5 in the case $u=0$ with the help of the implicit function theorem. Indeed, according to Lemma 5.5, there exists $\epsilon>0$ with the property that when $\left|c_{0}\right|<\epsilon$, there is a unique, small solution $\tau \equiv \tau_{c_{0}}$ to (5.11) for the case $u \equiv 0$ and $f \equiv c_{0}$. The corresponding map $q \equiv q_{c_{0}}$ in 
(5.10) is then pseudo-holomorphic. As the pair $\left(\zeta, c_{0}\right)$ vary, (5.10) defines a map, $\sigma$, from a neighborhood of the origin in $\mathbb{C}^{2}$ to $\mathbb{R}^{4}$. Thus, $\sigma\left(\zeta, c_{0}\right) \equiv q_{c_{0}}(\zeta)$. The inverse function theorem asserts that $\sigma$ is a diffeomorphism on some neighborhood of 0 in $\mathbb{C}^{2}$ if its differential at 0 is invertible. This will be the case, and Lemma 5.4 will follow if $\left|\frac{\partial \tau_{c_{0}}}{\partial c_{0}}\right|<1$ at $\left(\zeta, c_{0}\right)=0$. The latter inequality is insured by assertion (4) of Lemma 5.5 when $\rho$ is small.

(e) A local intersection number. This subsection provides the principal tool for studying $C$, that being Proposition 5.6, below, which asserts that $C$ has positive intersection number with certain embedded disks.

The statement of Proposition 5.6 requires a preliminary digression. To start the digression, fix a point $x \in C$ and fix a Gaussian coordinate system $\varphi: \mathbb{R}^{4} \rightarrow X$ which is centered at $x$ and which has $\left.\varphi^{*} \omega\right|_{x}=\omega_{0}$, where $\omega_{0} \equiv d x_{1} \wedge d x_{2}+d x_{3} \wedge d x_{4}$. As in the previous section, use the Euclidean metric and $\omega_{0}$ to identify $\mathbb{R}^{4}$ with $\mathbb{C}^{2}$ with coordinates $\left(w_{0}, w_{1}\right)$. The map $\varphi$ is an embedding on some ball $B \subset \mathbb{C}^{2}$ with center 0 , and with this understood, use $\varphi$ to identify $B$ with $\varphi(B)$. (This identification will now be implicit.) Fix $\rho>0$ and let $D$ denote the disk with radius $\rho$ and center 0 in $\mathbb{C}$. Make $\rho$ small enough so that $D \times D \subset B$.

Each point $w \subset D$ labels the disk $D_{w}$ in (5.11). For small $\rho$, the map $\Theta$ in Lemma 5.4 is defined. Consider, for each $w \in D$, the sequence

$$
\left\{\int_{D_{w}} \frac{1}{2 \pi} \Theta^{*} F_{A_{n}}\right\}_{n=1,2, \ldots} .
$$

Proposition 5.6. Suppose that $C$ intersects the closure of $\Theta\left(D_{0}\right)$ only at the origin. Then

(1) For all $w$ in a neighborhood of 0, the sequence in (5.16) converges.

(2) The limit is independent of $w$ in a neighborhood of 0.

(3) The limit, $m$, is an integer which is greater than zero.

(4) Let $H:[0,1] \times D_{0} \rightarrow D \times D$ be a smooth map with $\left.H(0, \cdot) \equiv \Theta\right|_{D_{0}}$ and with $C$ 's intersection with the closure of Image $(H)$ inside Image $(H)$. Then

$$
\left\{\int_{D_{0}} \frac{i}{2 \pi} H(1, \cdot)^{*} F_{A_{n}}\right\}_{n=1,2, \ldots}
$$

also converges with limit $m$.

The rest of this subsection is occupied with the proof of this proposition.

Proof of Proposition 5.6. Only the positivity of $m$ in the third assertion is hard. For the rest, remark first that $C$ is closed and that $C$ intersects $\Theta\left(D_{0}\right)$ only at the origin. Thus, there exists $\epsilon_{1}$ such that the intersection of $C$ with $V_{\epsilon_{1}} \equiv \bigcup_{w:|w| \leq \epsilon_{1}} \Theta\left(D_{w}\right)$ is compact.

Therefore, it follows from (5.7) and Stokes' theorem that the integrals of $\frac{i}{2 \pi} \Theta^{*} F_{A_{n}}$ over $D_{w}$ and $D_{w^{\prime}}$ are equal to within a factor of $z_{1} \cdot r_{n} \cdot \exp \left(-z_{1}^{-1} r_{n}^{1 / 2}\right)$ if $|w|+\left|w^{\prime}\right|<$ $\epsilon_{1}$. (Given the validity of the first assertion of Proposition 5.6, this last remark implies the second assertion of the proposition.) The approximate $w$-independence of the integrals of $\frac{i}{2 \pi} \Theta^{*} F_{A_{n}}$ over $D_{w}$ implies that the integral of $\frac{i}{2 \pi} F_{A_{n}}$ over $\Theta^{*} D_{0}$ is equal (within the small factor of $z_{1} \cdot r_{n} \cdot \exp \left(-z_{1}^{-1} \cdot r_{n}^{1 / 2}\right)$ ) to the integral of $-\frac{1}{2 \pi^{2} \epsilon_{1}^{2}} \Theta^{*} F_{A_{n}} \wedge d w_{0} \wedge d w_{0}^{*}$ over $V_{\epsilon_{1}}$. And, the latter integral converges as $n$ tends to $\infty$ because the sequence of currents $\left\{\mathcal{F}_{n}\right\}$ is assumed to converge. Thus assertions 
(1) and (2) of Proposition 5.6 have been proved. Assertion (4) follows from (5.7) and Stokes' theorem.

To prove that the limit $m$ is an integer, identify $D_{0}$ as a disk of radius $\rho$ in $S^{2}$. Since $\left|\nabla_{\Theta^{*} A_{n}}\left(\Theta^{*} \alpha_{n}\right)\right| \leq z \cdot r_{n} \cdot \exp \left(-\frac{\rho \cdot r_{n}^{1 / 2}}{z}\right)$ near the boundary of the $D_{0}$ (courtesy of Proposition 4.4), the restriction of $\Theta^{*} A_{n}$ to $D_{0}$ extends over $S^{2}$ as a smooth connection, $A^{\prime}$, on a complex line bundle over $S^{2}$ whose curvature is bounded in size by $\mathcal{O}\left(r_{n} \cdot \exp \left(-\frac{\rho \cdot r_{n}^{1 / 2}}{z}\right)\right)$ away from $D_{0}$. The first Chern number of this line bundle (an integer) is the integral over $S^{2}$ of $\frac{i}{2 \pi} F_{A^{\prime}}$, which is within $\mathcal{O}\left(r_{n} \cdot \exp \left(-\frac{\rho \cdot r_{n}^{1 / 2}}{z}\right)\right)$ of the integral of $\frac{i}{2 \pi} \Theta^{*} F_{A_{n}}$ over $D_{0}$.

The remainder of this subsection proves that Proposition 5.6's integer $m$ is positive. This part of the proof of assertion (3) of Proposition 5.6 is accomplished in four steps.

Step 1. This step introduces the key lemma that controls the integrals of $\frac{i}{2 \pi} \Theta^{*} F_{A_{n}}$ over pseudo-holomorphic disks (such as one from Lemma 5.4).

Lemma 5.7. Let $E \rightarrow X$ be a complex line bundle. There exists a constant $z$ which depends only on $c_{1}(E)$ and on the Riemannian metric and which has the following significance: For $r \geq 1$, let $(A,(\alpha, \beta))$ be a solution to (1.20) for the $\mathrm{Spin}^{\mathbb{C}}$ structure in (1.14). Let $\Sigma \subset X$ be a pseudo-holomorphic submanifold. Then the pull-back to $\Sigma$ of $\frac{i}{2 \pi} F_{A}$ involves only the terms

$$
\frac{1}{16 \pi}\left(1-|\alpha|^{2}+|\beta|^{2}\right) \cdot \omega+\frac{i}{2 \pi} P_{-} F_{A} .
$$

Furthermore, the restriction of (5.17) to $\Sigma$ has the form $h \cdot d \operatorname{vol}_{\Sigma}$, where $d \operatorname{vol}_{\Sigma}$ is the area 2-form from the induced metric, and where $h$ is a function on $\Sigma$ which is bounded from below by $-z$.

Proof of Lemma 5.7. This statement is true if true at each point of $\Sigma$. Let $x \in \Sigma$ and let $\left(w_{0}, w_{1}\right)$ be (complex) Gaussian coordinates near $x$ as in the previous section (so $x$ corresponds to the origin in $\mathbb{C}^{2}$ and $\left.\omega\right|_{x}=\omega_{0}$ ). Note that the almost complex structure $J$ on $T X$ restricts to $x$ as the given complex structure $\left.T \mathbb{C}^{2}\right|_{0}$. Thus, the assertion that $\Sigma$ is pseudo-holomorphic implies that $\left(w_{0}, w_{1}\right)$ can be chosen so that $\left.T \Sigma\right|_{x}$ be annihilated by $\frac{i}{2} d w_{0} \wedge d w_{0}^{*}$. The terms in (1.20) which involve $\alpha \beta^{*}$ and $\alpha^{*} \beta$ restrict at $x$ as linear combinations of $d w_{0} \wedge d w_{1}$ and its complex conjugate. Thus, these restrict as 0 to $\Sigma$ at $x$. This proves the first assertion of the lemma. The second assertion follows from Proposition 3.4

Step 2. This step introduces a useful family of pseudo-holomorphic perturbations of the disk $\Theta\left(D_{w}\right)$. To begin, remark that given $\epsilon>0$, there are, for all $n$ sufficiently large, points of $\alpha_{n}^{-1}(0)$ which have distance $\epsilon$ or less to the point $x$.

With $\epsilon>0$ chosen small and $n$ chosen large, focus attention on a point $x^{\prime} \epsilon$ $\alpha_{n}^{-1}(0)$ which is very close to $x$. Assume that $\operatorname{dist}\left(x^{\prime}, x\right)<\epsilon$. The point $x^{\prime}$ lies in $\Theta\left(D_{w}\right)$ for some $w \in \mathbb{C}$ with $|w|<\epsilon$.

Fix complex Gaussian coordinates $\left(w_{0}^{\prime}, w_{1}^{\prime}\right)$, centered at $x^{\prime}$. There is a unique complex line $\Pi_{0}$ through 0 in the $\left(w_{0}^{\prime}, w_{1}^{\prime}\right)$ version of $\mathbb{C}^{2}$ which is tangent to $\Theta\left(D_{w}\right)$ at 0 . (Remember that $\Theta\left(D_{w}\right)$ is pseudo-holomorphic so holomorphic at 0 in the $\left(w_{0}^{\prime}, w_{1}^{\prime}\right)$ version of $\mathbb{C}^{2}$.) The line $\Pi_{0}$ can be taken as the set where $w_{1}^{\prime} \equiv 0$.

Given $\kappa \in \mathbb{C}$, use $\Pi_{\kappa}$ to denote the complex line in the $\left(w_{0}^{\prime}, w_{1}^{\prime}\right)$ version of $\mathbb{C}^{2}$ where $w_{1}^{\prime}=\kappa w_{0}^{\prime}$. Given $\delta>0$, use $\Omega \subset \mathbb{C P}^{1}$ to denote the subset of complex lines 
$\Pi_{\kappa}$ with $|\kappa|<\delta$. The next lemma asserts that when $\delta$ is small, then each complex line in $\Omega$ parameterizes a pseudo-holomorphic perturbation of $\Theta\left(D_{w}\right)$ through $x^{\prime}$.

Lemma 5.8. There exists $z>0$ which depends only on the Riemannian metric and on $\omega$; and there exists $\delta_{0}>0$ and $\epsilon_{0}>0$ which depend on the point $x$. These have the following property: Require that the distance from $x^{\prime}$ to $x$ be less than $\epsilon_{0}$. Take $\delta<\delta_{0}$ and then define $\Omega$ as above. Let $D_{2} \subset D$ denote the disk with radius $\frac{\rho}{2}$. There is a J-pseudo-holomorphic map $\Theta_{0}: D_{2} \times \Omega \rightarrow D \times D$ with the following properties:

(1) $\Theta_{0}(\cdot, 0) \equiv \Theta(\cdot, w)$.

(2) Write $\Theta^{-1}\left(x^{\prime}\right) \equiv\left(\zeta_{0}, w\right)$. Then $\Theta_{0}\left(\zeta_{0}, \kappa\right) \equiv x^{\prime}$ for all $\kappa \in \Omega$.

(3) $\Theta_{0}\left(D_{2}, \kappa\right)$ is tangent to the plane $\Pi_{\kappa}$ at $x^{\prime}$.

(4) The second derivatives of $\Theta_{0}(\cdot, \kappa)$ are bounded by $z$.

(5) $C$ intersects the closure of $\Theta_{0}\left(D_{2}, \kappa\right)$ in $\Theta_{0}\left(D_{2}, \kappa\right)$ for all $\kappa \in \Omega$.

Proof of Lemma 5.8. To ease the notation, take $x^{\prime}=\Theta(0, w)$. Then, the map $\Theta(\cdot, w)$ has the form $\zeta \rightarrow(\zeta, u(\zeta))$ in the $\left(w_{0}^{\prime}, w_{1}^{\prime}\right)$ coordinates. With this understood, look for $\Theta_{0}(\cdot, \kappa)$ to be a map which sends $\zeta$ to $q(\zeta) \equiv\left(\zeta, u(\zeta)+\left(\kappa+\eta_{0}\right) \cdot \zeta\right.$ $+\eta_{1}+\tau(\zeta)$ ), where $\eta_{0,1} \in \mathbb{C}$ will be determined by the constraints of assertions (2) and (3). With the preceding understood, now apply Lemma 5.5 to find that when $|\kappa|,\left|\eta_{0}\right|$ and $\left|\eta_{1}\right|$ are all small, there is a unique solution $\tau \equiv \tau\left[\kappa, \eta_{0}, \eta_{1}\right]$ to (5.11) with $\|\tau\| \leq z \cdot\left(\rho^{2}\left(|\kappa|+\left|\eta_{0}\right|\right)+\rho\left|\eta_{1}\right|\right)$. Lemma 5.5 insures that $\Theta_{0}(\cdot, \kappa)$ is pseudo-holomorphic.

Now, assertion (1) is satisfied by construction. To obtain assertions (2) and (3), fix $\kappa$ and let $\left(\eta_{0}, \eta_{1}\right)$ vary over a small ball $B_{\eta} \subset \mathbb{C}^{2}$ about 0 to define a smooth map $\sigma: B_{\eta} \rightarrow \mathbb{C} \times \mathbb{C P}^{1}$ which associates to each pair $\left(\eta_{0}, \eta_{1}\right)$ the following pair: First, the $w_{1}$ coordinate of the $q(\zeta=0)$, with $q$ as above (and see (5.10)). Second, the tangent plane to the image of $q$ at $q(\zeta=0)$. That is, $\sigma\left(\eta_{0}, \eta_{1}\right)=\left(\eta_{1}+\tau(0),\left[1, \kappa+\eta_{0}+\tau^{\prime}\right]\right.$, where $\left.\tau^{\prime} \equiv \frac{\partial \tau}{\partial \zeta}\right|_{\zeta=0}$. Now invoke assertion (4) of Lemma 5.5 and the implicit function theorem to choose $\left(\eta_{0}, \eta_{1}\right)$ as smooth functions of $\kappa$ (with $\left|\eta_{0}\right| \leq z \cdot \rho|\kappa|$ and $\left|\eta_{1}\right| \leq$ $\left.z \cdot \rho^{2} \cdot|\kappa|\right)$ so that $\sigma\left(\eta_{0}, \eta_{1}\right) \equiv(0,[1, \kappa])$. Assertions (2) and (3) of Lemma 5.8 are therefore satisfied with these choices for the pair $\left(\eta_{0}, \eta_{1}\right)$.

Assertion (4) of Lemma 5.8 follows from Lemma 5.5 directly. And, assertion (5) follows for suitable $\epsilon$ and $\delta_{0}$ because $C \subset X$ is closed.

Step 3. Let $x^{\prime}$ be as in the preceding step. This step finds a disk $\mathfrak{D} \equiv \Theta_{0}\left(D_{2}, \kappa\right)$ as above which intersects $\alpha_{n}^{-1}(0)$ at $x^{\prime}$ in a nice way. To begin, observe that according to Proposition 4.2, there is no generality lost in assuming that after rescaling (as in (4.6)), the data $\left(\underline{A}_{n},\left(\underline{\alpha}_{n}, \underline{\beta}_{n}\right)\right)$ is within $8^{-n}$ in the $C^{k}$ norm on the radius $2^{n}$ ball about 0 of some solution $\left(\bar{A}_{0}, \alpha_{0}\right)$ of the Seiberg-Witten equations on $\mathbb{C}^{2}$ (this is the $\left(w_{0}^{\prime}, w_{1}^{\prime}\right)$ version of $\left.\mathbb{C}^{2}\right)$. Here, one can assume that $2^{n} \cdot r_{n}^{-1 / 2} \ll 2^{-n}$. The integer $k$ is determined as follows: Remember that $\alpha_{0}^{-1}(0)$ is an algebraic curve through 0 in $\mathbb{C}^{2}$, of a priori bounded degree; and with this understood, one can fix any value of $k$ which is, say, ten times this a priori bound.

The fact that $\alpha_{0}^{-1}(0)$ is the zero set of a polynomial with an a priori bounded degree implies that the completed curve in $\mathbb{C P}^{2}$ intersects the $\mathbb{C P}^{1}$ at $\infty$ in $\mathbb{C}^{2}$ in a finite set with an a priori bound on the number of elements. This number, $z_{1}$, depends only on $c_{1}(E)$ and on the Riemannian metric. The existence of such a bound has the following consequence: Given $\delta_{1}$, there are some $z_{1}$ disks in $\mathbb{C P}^{1}$ of radius $\delta_{1}$ such that any complex line $\Pi \in \mathbb{C}^{2}$ through 0 whose parameter is outside 
all of these disks has the following property: Any point $\zeta \equiv\left(w_{0}^{\prime}, w_{1}^{\prime}\right)$ in $\Pi$ whose distance from the origin is $2^{n}$ obeys

$$
\operatorname{dist}\left(\zeta, \alpha_{0}^{-1}(0)\right) \geq \frac{\delta_{1}}{z_{2}} 2^{n} .
$$

Here, $z_{2}$ depends on the a priori degree bound (that is, on $c_{1}(E)$ and the Riemannian metric). These $z_{1}$ disks will be called bad disks.

With $z_{1}$ as above, set $\delta_{1} \equiv \frac{\delta_{0}}{z_{1} \cdot 10^{3}}$.

Step 4 . Let $\Omega$ be as described in Lemma 5.8 and pick $\kappa \in \Omega$ which avoids all bad disks. Write $\Theta^{-1}\left(x^{\prime}\right)$ as $\left(\zeta_{0}, w\right)$ and let $D^{\prime \prime} \subset \mathbb{C}$ denote the disk with center $\zeta_{0}$ and radius $\frac{2^{n}}{r_{n}^{1 / 2}}$. Then, the integral of $\frac{i}{2 \pi} F_{A_{n}}$ over $\Theta_{0}\left(D^{\prime \prime}, \kappa\right)$ can be computed by comparing it to the integral of $\frac{i}{2 \pi} F_{A_{0}}$ over the rescaled version in $\mathbb{C}^{2}$ (via (4.6)) of $\Theta_{0}\left(D^{\prime \prime}, \kappa\right)$. The latter integral is computed using Proposition 4.1: It is within $\exp \left(-\frac{\delta}{z_{2} \cdot 2^{n}}\right)$ of a positive integer.

Meanwhile, the integral of $\frac{i}{2 \pi} F_{A_{0}}$ over the rescaled (via (4.6)) version of $\Theta_{0}\left(D^{\prime \prime}, \kappa\right)$ is within $8^{-n} \pi \cdot\left(2^{n}\right)^{2}=z_{3} \cdot 4^{-n}$ of the integral over the same disk of $\frac{i}{2 \pi} F_{\underline{A}_{n}}$. (Here, remember that $\underline{A}_{n}$ is obtained from $A_{n}$ by rescaling with (4.6).) Thus, the integral of $\frac{i}{2 \pi} F_{A_{n}}$ over $\Theta_{0}\left(D^{\prime \prime}, \kappa\right)$ has the form

$$
m_{0}+\mathcal{O}\left(4^{-n}+\exp \left(-\frac{\delta_{1}}{z_{2} 2^{n}}\right)\right)
$$

where $m_{0}$ is a positive integer.

To estimate the value of the integral over the remainder of $\Theta_{0}\left(D_{2}, \kappa\right)$, apply Lemma 5.7 to conclude that the integral over the remainder can be no smaller than $-z_{3} \cdot \rho^{2}$. One therefore concludes that the integral of $\frac{i}{2 \pi} F_{A_{n}}$ over the whole of $\Theta_{0}\left(D_{2}, \kappa\right)$ is no smaller than

$$
1-z_{3} \cdot\left(4^{-n}+\rho^{2}\right) \geq \frac{1}{2}
$$

if $\rho$ is small.

This last estimate implies the positivity of the limit $m$ from Proposition 5.6.

\section{Positivity And PSEUdo-Holomorphic CuRVES}

This section applies the intersection theoretic result from Proposition 5.6 to prove that $C$ is the image of a pseudo-holomorphic curve. The basic strategy dates back to $[\mathrm{Ki}]$ at least. To understand the strategy, one should understand first a proof of (1.23). For a proof, use Taylor's theorem with remainder to represent $\Sigma$ near a point as graph over its tangent space. If the tangent space is not complex at the given point, then it is easy to write down a linear $\mathbb{C P}^{1}$ which has negative intersection number with $\Sigma$ at the point.

Proposition 6.1, below, concerns a set $C$, in a compact, symplectic 4-manifold $X$, with some special homological properties. Subsection 6a, below, supplies Proposition 6.1 and discusses its relevance to $C$ from (5.6). Modulo the proof of Proposition 6.1 (which occupies the remainder of this section), the proof of Theorem 1.3 is completed in the first subsection.

The remaining subsections prove Proposition 6.1 in stages. Subsection $6 \mathrm{~b}$ proves that $C$ has locally the structure of a continuous, multi-valued graph over a disk. Subsection $6 \mathrm{c}$ proves that $C$ is, almost everywhere, a Lipschitz submanifold of $X$. 
Subsection $6 \mathrm{~d}$ then proves that $C$ is, almost everywhere, a pseudo-holomorphic submanifold. The last subsection considers $C$ 's singularities and completes the proof of Proposition 6.1.

(a) Positive cohomology assignments. A preliminary three part digression is required to state Proposition 6.1. For the purposes of the digression, let $C \subset X$ be a set. For Part 1 of the digression, let $D \subset \mathbb{C}$ denote the standard unit disk. A map $\sigma: D \rightarrow X$ will be called admissible when $C$ intersects the closure of $\sigma(D)$ inside $\sigma(D)$. Part 2 of the digression introduces the notion of a positive cohomology assignment to $C$. This is an assignment of an integer, $I(\sigma)$, to each admissible map $\sigma: D \rightarrow X$. But, the following criteria have to be met:

(1) If $\sigma: D \rightarrow X-C$, then $I(\sigma)=0$.

(2) If $\sigma_{0}, \sigma_{1}: D \rightarrow X$ are admissible and homotopic via an admissible homotopy (a homotopy $h:[0,1] \times D \rightarrow X$ where $C$ intersects the closure of Image $(h)$ inside Image $(h))$, then $I\left(\sigma_{0}\right)=I\left(\sigma_{1}\right)$.

(3) Let $\sigma: D \rightarrow X$ be admissible and let $\theta: D \rightarrow D$ be a proper, degree $k$ map. Then $I(\sigma \circ \theta)=k \cdot I(\sigma)$.

(4) Suppose that $\sigma: D \rightarrow X$ is admissible and that $\sigma^{-1}(C)$ is contained in a disjoint union $\bigcup_{\nu} D_{\nu} \subset D$ where each $D_{\nu} \equiv \theta_{\nu}(D)$ with $\theta_{\nu}: D \rightarrow D$ being an orientation preserving embedding. Then $I(\sigma)=\sum_{\nu} I\left(\sigma_{\nu}\right)$.

(5) If $\sigma: D \rightarrow X$ is admissible and a pseudo-holomorphic embedding with $\sigma^{-1}(C) \neq \varnothing$, then $I(\sigma)>0$.

Part 3 of the digression considers the following example: Let $\Sigma$ be a compact, complex curve and let $\varphi: \Sigma \rightarrow X$ be a pseudo-holomorphic map. Here is a positive cohomology assignment for $C \equiv \varphi(\Sigma)$ : When $\sigma: D \rightarrow X$ is admissible, there are arbitrarily small perturbations of $\sigma$ which produce a map $\sigma^{\prime}$ which is homotopic to $\sigma$ through admissible maps and which is transversal to $\varphi$. The fiber product $T \equiv\left\{(x, y) \in D \times \Sigma: \sigma^{\prime}(x)=\varphi(y)\right\}$ is thus a smooth, oriented, 0-dimensional submanifold of $D \times \Sigma$; that is, $T$ is a finite set of signed points. Declare $I(\sigma)$ to be the sum of these signs.

End the digression.

Proposition 6.1. Let $X$ be a compact, symplectic 4-manifold with compatible almost complex structure and let $C \subset X$ be a closed set with finite 2-dimensional Hausdorff measure and a positive cohomology assignment. Then there is a compact, complex curve $\Sigma$ and a pseudo-holomorphic map $\varphi: \Sigma \rightarrow X$ such that $C=\varphi(\Sigma)$. Furthermore, the positive cohomology assignment for $C$ is identical to that described above for the pair $(\varphi, \Sigma)$.

In the case of $C$ from (5.6), a positive cohomology assignment is obtained via Proposition 5.6. The following lemma states the case.

Lemma 6.2. Let $C$ be as in (5.6) and Lemma 5.2 and let $\sigma: D \rightarrow X$ be an admissible map. Then $\left\{\int_{D} \frac{i}{2 \pi} \sigma^{*} F_{A_{n}}\right\}_{n=1,2, \ldots}$ converges to an integer, $I(\sigma)$. And, the assignment of $I(\sigma)$ to such an admissible $\sigma$ defines a positive cohomology assignment. 
Proof of Lemma 6.2. Assuming that the limit exists, we argue as in the proof of Proposition 5.6 to establish properties (1)-(4) in (6.1). To find the limit, first perturb $\sigma$ slightly so that the resulting disk is immersed. Then, use [Fe, 2.10.27] to conclude that there exists such a perturbation that intersects $C$ in a finite set of points, none of which are double points of the immersion. (The reference to Federer's book is, in the present context, the plausible assertion that a set of Hausdorff dimension 2 in a 4-manifold will have finite intersection with almost every fiber of a fibration over a 2-dimensional disk.) A small disk in $D$ centered in $\sigma^{\prime-1}(C)$ will contain only one such point, so it is enough to establish the limit $\Theta(\sigma)$ for an embedded disk which intersects $C$ only once, in its interior. The existence of this limit follows now by the argument which proves assertion (1) of Proposition 5.6. The proof that Proposition 5.6's limit $m$ is an integer proves that this $I(\sigma)$ is an integer here too. The fifth assertion of (6.1) is obtained from Proposition 5.6.

Proof of Theorem 1.3. It follows from Proposition 6.1 and Lemma 6.2 that there is a compact, complex curve $\Sigma$ and a pseudo-holomorphic map $\varphi: \Sigma \rightarrow X$ such that $\varphi(\Sigma)=C$ with $C$ from (5.6). By passing to a multiple covering over components of $\Sigma$ (if necessary), one can arrange for $\varphi_{*}[\Sigma]$ to equal the Poincaré dual of $c_{1}(E)$.

With the preceding understood, the theorem is proved with a demonstration that the given sets $\left\{\Omega_{i}\right\}$ intersect $C$. For this, take $i$ and note that $\Omega_{i} \cap U(N) \neq \varnothing$ for all $N$. Since $\Omega_{i}$ is closed, $\Omega_{i}$ is therefore compact, so $\Omega_{i} \cap\left(\bigcap_{N} U(N)\right) \neq \varnothing$. Thus, $\Omega_{i} \cap C \neq \varnothing$.

(b) Continuity. In this and all subsequent subsection, the set $C \subset X$ will be as described in Proposition 6.1. This section begins the proof of Proposition 6.1 by establishing a preliminary regularity result for $C$. Lemma 6.3 , below, summarizes. The statement of the lemma requires a short digression to discuss some features of complex Gaussian coordinates.

To begin the digression, recall that complex Gaussian coordinates at a point $x \in X$ are, first of all, Gaussian coordinates $\left(x_{1}, x_{2}, x_{3}, x_{4}\right)$ written as $\left(w_{0}=x_{1}+\right.$ $\left.i x_{2}, w_{1}=x_{3}+i x_{4}\right)$ for $\mathbb{C}^{2}=\mathbb{R}^{4}$. They are constrained so that $\left.\omega\right|_{x}=\omega_{0}=$ $\frac{i}{2}\left(d w_{0} \wedge d w_{0}^{*}+d w_{1} \wedge d w_{1}^{*}\right)$. The set of complex coordinates at $x$ has naturally the structure of a principal $\mathrm{U}(2)$ bundle over a point. (As $x$ varies over $X$, one obtains the $J$-induced principal U(2) bundle reduction of the orthonormal frame bundle of $X$.)

Note that any two such complex coordinates at $x$ define the same complex structure $J_{0}$ on $\mathbb{C}^{2}$. (This is the restriction of $J$ to the tangent space to $X$ at $x$.) In any event, there is an unambiguous notion of a complex line through the origin, a notion which is defined independently of the choice of the complex coordinates. However, each complex coordinate system determines a unique complex line, the one where $w_{1}=0$.

At this point, recall that $\mathbb{C P}^{1}$ parameterizes the space of complex lines through the origin in $\mathbb{C}^{2}$. The map from complex Gaussian coordinate systems to complex lines is simply the projection $\mathrm{U}(2) \rightarrow \mathrm{U}(2) /(\mathrm{U}(1) \times \mathrm{U}(1))=\mathbb{C P}^{1}$. With the preceding understood, a complex Gaussian coordinate system $\left(w_{0}, w_{1}\right)$ will be called compatible with a given complex line if said line is given by the equation $w_{1}=0$.

Lemma 6.3. Let $x \in C$. There exists a full measure subset of $\mathbb{C P}^{1}$ for which any compatible, complex Gaussian coordinates $\left(w_{0}, w_{1}\right)$ centered at $x$ has the following property: There are numbers $\rho>0$ and $\epsilon \in(0, \rho)$, and there is a positive integer $m$. 
Given this data, let $D \equiv\{\zeta \in \mathbb{C}:|\zeta|<\rho\}$ and let $D^{\prime} \subset D$ denote the concentric disk with radius $\epsilon$. Then, $\Theta$ from Lemma 5.4 is defined on $D \times D^{\prime}$. And, there is a continuous map, $\Phi$, from $D^{\prime}$ to the space of unordered $m$-tuples of points in $D$ such that

$$
\Theta^{-1}(C)=\left\{(\zeta, w): w \in D^{\prime} \text { and } \zeta \in \Phi(w)\right\} .
$$

(The space of unordered $m$-tuples of points in $D$ is topologized as the quotient of $\times_{m} D$ by the obvious action of the symmetric group on $m$ letters. This space has the structure of a stratified space (with smooth strata). The top dimensional stratum has all $m$ distinct points; the smallest dimensional stratum has all $m$ points coincide, it is a copy of $D$.)

The result is a direct consequence of the next two lemmas:

Lemma 6.4. Let $\rho_{0}$ and $\Theta$ be as in Lemma 5.4. Let $x \in C$ and, for positive $\rho<\rho_{0}$, let $\left\{D_{w}\right\}$ be as in (5.11). Suppose that $C \cap \Theta\left(D_{0}\right)=x$. Then there exists $\epsilon>0$ such that $C \cap D_{w}$ is a finite set of points if $|w|<\epsilon$.

To put this lemma in context, recall that Lemma 5.4 associates to each complex Gaussian coordinate system centered at $x$ a number $\rho>0$ and a map $\Theta$. Now, consider

Lemma 6.5. Let $x \in C$. There is a set of full measure in $\mathbb{C P}^{1}$ whose compatible, complex Gaussian coordinates are such that $\Theta\left(D_{0}\right) \cap C$ is a finite set of points.

The proof of this lemma requires a preliminary digression to discuss a perturbation result about pseudo-holomorphic disks through a fixed point. To begin the digression, consider a point $x \in X$ and some complex Gaussian coordinates $\left(w_{0}, w_{1}\right)$ centered at $x$. For $\rho>0$, let $E \rightarrow \mathbb{C P}^{1}$ denote the radius $\rho$ disk bundle in the complex line bundle with first Chern class -1 . With the coordinates $\left(w_{0}, w_{1}\right)$ chosen, this bundle $E$ has a natural identification with the space of pairs $(\zeta, \Pi)$, where $\Pi$ is a complex line and where $\zeta$ is a point on $\Pi$.

In general, a complex line $\Pi$ as above will not be $J$-pseudo-holomorphic (except at the origin). With this understood, consider:

Lemma 6.6. There exist constants $z$ and $\rho_{0}>0$ with the following significance: Fix $\rho<\rho_{0}$ and let $E_{\rho} \subset E \rightarrow \mathbb{C P}^{1}$ denote the disk subbundle of pairs $(\zeta, \Pi)$ with $|\zeta|<\rho$. There exists a smooth map $\Theta_{0}: E_{\rho} \rightarrow \mathbb{C}^{2}$ with:

(1) For each $\kappa \in \mathrm{U}_{0}$, the map $\Theta_{0}:\left.E_{\rho}\right|_{\kappa} \rightarrow \mathbb{C}^{2}$ is pseudo-holomorphic.

(2) $\Theta_{0}$ maps the zero section $\underline{0}$ to 0 and $\Theta_{0}$ embeds $E_{\rho}-\underline{0}$.

(3) For each $\kappa \in \mathbb{C P}^{1}$, the disk $\Theta_{0}\left(\left.E_{\rho}\right|_{\kappa}\right)$ is tangent to $\Pi_{\kappa}$ at the origin.

(4) The second derivatives of $\Theta_{0}$ are bounded by $z$.

Proof of Lemma 6.6. Mimic the proof of Lemma 5.8.

With Lemma 6.6 in hand, consider the

Proof of Lemma 6.5. Take $x \in C$ and construct the map $\Theta_{0}$ as described in Lemma 6.6. Refer to $[\mathrm{Fe}, 2.10 .27]$ to conclude that for each positive integer $N$, there is a subset of $\kappa \in \mathbb{C P}^{1}$ of full measure with the property that $\Theta_{0}^{-1}(C)$ intersects $\left\{\left.\zeta \in E_{\rho}\right|_{\kappa}:|\zeta|>N^{-1}\right\}$ as a finite set. Finally, note that a countable union of sets of measure zero has measure zero. 
Proof of Lemma 6.3 (assuming Lemmas 6.4 and 6.5). Suppose that $x \in C$ and complex Gaussian coordinates are chosen (centered at $x$ ) so that $\Theta\left(D_{0}\right)$ is defined for some $\rho>0$. (There exists a full measure set of these according to Lemma 6.5.) Then $x$ is an isolated point of $C \cap \Theta\left(D_{0}\right)$ which means that there exists $\rho^{\prime} \in(0, \rho)$ such that $x$ is the only point of intersection between $C$ and the $\Theta$ image of the disk $D_{0}^{\prime} \subset D_{0}$ with radius $\rho^{\prime}$ and center 0 . Now, let $D_{w}^{\prime} \subset D_{w}$ denote the concentric disk with radius $\rho^{\prime}$. Thus, by Lemma 6.4 , the intersection of $C$ with $\Theta\left(D_{w}^{\prime}\right)$ contains no more than $m \equiv I\left(\left.\Theta\right|_{D_{0}^{\prime}}\right)$ points. Furthermore, $I(\cdot)$ assigns a positive integer to each such point and the sum of these integers must equal $m$ (see (6.1)(4) and (6.1)(5)). This gives the map $\Phi$. The continuity of $\Phi$ is a consequence of the fact that $C$ is closed.

The remainder of this subsection is occupied with the

Proof of Lemma 6.4. Because $C$ is compact, given $\delta \in(0, \rho)$, there exists $\epsilon>0$ with the following significance: Let $D_{w}^{\prime} \subset D_{w}$ denote the concentric disk of radius $\delta$. Then $C \cap \Theta\left(D_{w}\right) \subset \Theta\left(D_{w}^{\prime}\right)$. And, (6.1)(4) and (6.1)(5) insure that $C \cap \Theta\left(D_{0}\right)$ has no more than $m \equiv I\left(\left.\Theta\right|_{D_{0}}\right)$ components. Suppose, however, that there were, for some $w$ with $|w|$ small, some $m+1$ points in this intersection. The plan is to generate a contradiction from this assumption. The contradiction is obtained by constructing an admissible map of a disk into $X$ which has the following properties: First, it is a perturbation of $\Theta\left(\left.\right|_{D_{w}}\right)$ (through admissible disks). Second, its image intersects $C$ in embedded sub-disks which are pseudo-holomorphic. Finally, its image intersects $C$ in at least $m+1$ components. Then, (6.1)(2) will disagree with $(6.1)(4)$ and $(6.1)(5)$ about the value of $I(\cdot)$ on this embedding, thus voiding the initial assumption.

To begin, let $d$ denote the diameter of the largest component of $C \cap \Theta\left(D_{w}\right)$. By assumption, $d>0$ and, given $\epsilon>0$, there exists $\delta>0$ such that when $|w|<\delta$ then $d<\varepsilon$. One can then find a subdisk $D^{\prime} \subset D_{w}$ of radius $\rho_{1}$ with $4 d<\rho_{1}<2^{4 m+4} \cdot d$ and with the following property: Let $D^{\prime \prime} \subset D^{\prime}$ denote the concentric disk of radius $\frac{1}{4} \rho_{1}$. Then $C \cap \Theta\left(D^{\prime}\right) \subset \Theta\left(D^{\prime \prime}\right)$ and this set contains $m+1$ points whose minimal mutual distance apart is at least $\frac{d}{m+2}$. Denote these points as $\left(\zeta_{i}, u\left(\zeta_{i}\right)\right)$, where $u: D_{0} \rightarrow \mathbb{C}$ is a complex valued function with $\|u-w\| \leq z \cdot \rho^{2}$ (see (5.15) and Lemma 5.4). No generality is lost (and notation is simplified) by assuming that the center of $D^{\prime}$ is the origin and that

$$
\left|\zeta_{i}\right|>\frac{d}{4(m+2)} .
$$

(If this condition is not satisfied, translate the origin of $\mathbb{C}$.)

Note that $\left.\Theta\right|_{D^{\prime}}$ is admissible, and $I\left(\left.\Theta\right|_{D^{\prime}}\right) \leq m$. With this understood, the plan is to find an admissible, pseudo-holomorphic perturbation of $\left.\Theta\right|_{D^{\prime}}$ which intersects $C$ in at least $m+1$ components. (This perturbation will go through each $\left(\zeta_{i}, u\left(\zeta_{i}\right)\right)$.) The perturbation in question will have the form of $q$ in (5.10) with

$$
f(\zeta) \equiv \eta \cdot \prod_{i}\left(\zeta-\zeta_{i}^{\prime}\right)
$$

for suitable choices of $\eta$ and $\left\{\zeta_{i}^{\prime}\right\}$. (Thus, $\eta \equiv 0$ is the unperturbed disk.)

To see how (6.4) fits into the strategy, note that the parameters $\left\{\zeta_{i}^{\prime}\right\}$ will be chosen to insure that the perturbed disk goes through each $\left(\zeta_{i}, u\left(\zeta_{i}\right)\right)$. Meanwhile, the parameter $\eta$ will be chosen to insure that the intersection of the perturbed disk 
with $C$ is discrete. The point is that the disks defined by differing $\eta$ in (6.4) will be seen to intersect in a discrete set (courtesy of Aronszajn's unique continuation principle [Ar].) With this understood, a basic measure theoretic fact [Fe, 2.10.27] will insure that there are $\eta$ 's arbitrarily close to zero for which the corresponding disk has discrete intersections with $C$. By the way, Lemma 5.5 guarantees that the embedded disk for any sufficiently small $\eta$ is defined by an admissible map which is homotopic to $\Theta\left(D^{\prime}\right)$ via an admissible homotopy. Thus $I\left(\left.\Theta\right|_{D^{\prime}}\right)$ is the same as that for the embedding defined by any sufficiently small $\eta$, which is larger than $m$-a contradiction.

To choose these parameters, remember that the set $\left\{\zeta_{i}^{\prime}\right\}$ is chosen to make

$$
f\left(\zeta_{i}\right)+\tau\left(\zeta_{i}\right)=0
$$

for all $i$. With $\eta$ fixed (for the time being) with small norm, (6.5) can be thought of as an equation for the set $\left\{\zeta_{i}^{\prime}\right\}$ and the plan is to search for a solution of the form $\zeta_{i}^{\prime}=\zeta_{i}+\mu_{i}$ with each $\mu_{i}$ very small. For this purpose, remark that (6.5) implies that when $\eta$ and $\mu \equiv\left\{\mu_{i}\right\}$ have small norm, then $\mu$ obeys

$$
\sum_{j} \frac{\partial f}{\partial \zeta_{j}^{\prime}}\left(\zeta_{i}\right) \cdot \mu_{j}+\tau\left(\zeta_{i}\right)+\mathcal{N}(\mu)=0,
$$

where $\mathcal{N}$ is the remainder term in an appropriate Taylor's expansion. Furthermore, Lemma 5.5 allows for the following estimate on $\tau\left(\zeta_{i}\right)$ and $\mathcal{N}$ :

$$
\begin{aligned}
& \text { (1) }\left|\tau\left(\zeta_{i}\right)\right| \leq c_{m}|\eta| d^{m+1}, \\
& \text { (2) }|\mathcal{N}(\mu)| \leq c_{m}|\eta|\left(d^{m-2}|\mu|^{2}+d^{m}|\mu|\right), \\
& \text { (3) }\left|\frac{\partial \mathcal{N}}{\partial \mu_{i}}\right| \leq c_{m}|\eta|\left(d^{m-3}|\mu|^{2}+d^{m-2}|\mu|\right) .
\end{aligned}
$$

Here, $c_{m} \leq z^{m}$ for some constant $z$. (Remember: $4 d \leq \rho_{1} \leq 2^{4 m+4} d$.)

Meanwhile, note that the matrix $M_{i j} \equiv \frac{\partial f}{\partial \zeta_{j}}\left(\zeta_{i}\right)$ is diagonal with its $i$ 'th eigenvalue equal to $\eta \prod_{j \neq 1}\left(\zeta_{i}-\zeta_{j}\right)$. Thus, the eigenvalues of $M_{i j}$ have absolute value greater than

$$
\frac{1}{c_{m}}|\eta| d^{m-1}
$$

The contraction mapping theorem with (6.7) and (6.8) finds $d_{m}>0$ (which depends on $m$ ) such that when $d<d_{m}$ and $|\eta|<d_{m}$, then there is a unique small solution $\mu \equiv \mu(\eta)$ to $(6.6)$ with the property that

$$
|\mu| \leq c_{m}^{\prime} d^{2} .
$$

Here, $c_{m}^{\prime}$ is a constant which depends on $m$.

Thus, for each choice of (small) $\eta$, the preceding constructs a map $\sigma_{\eta}: D^{\prime} \rightarrow X$ (given by (5.10)) which is a pseudo-holomorphic embedding. The next task is to examine how these $\sigma_{\eta}$ depend on the parameter $\eta$. In particular, the claim is that for sufficiently small $\eta$ and $\eta^{\prime}$, the images of $\sigma_{\eta}$ and $\sigma_{\eta^{\prime}}$ intersect in a discrete set. Here is the proof: Consider the map, $e_{0}$, of a small disk in $\mathbb{C}$ into $\mathbb{C}$ which sends $\eta$ to the $w_{1}$ coordinate of $\sigma_{\eta}(0)$. (The $w_{0}$ coordinate of $\sigma_{\eta}(0)$ is 0 .) This map is smooth (courtesy of Lemma 5.5). The value of this map at $\eta=0$ is $w$. And, its 
differential at 0 sends $\lambda \in \mathbb{C}$ to

$$
\lambda \cdot \prod_{j}\left(-\zeta_{j}^{\prime}\right)+\mathcal{O}\left(c_{m} d^{m+1}\right)|\lambda| .
$$

The error term here follows from Lemma 5.5 too. Thus (6.3) and (6.10) insure that the differential of the map $e_{0}$ is surjective at $\eta=0$. This means, in particular, that the map $e_{0}$ is a diffeomorphism onto its image on a small disk $U \subset \mathbb{C}$ containing 0 . So, if $\eta$ and $\eta^{\prime}$ are distinct points in $U$, the maps $\sigma_{\eta}$ and $\sigma_{\eta^{\prime}}$ do not share the same range. Thus, by Aronszajn's theorem [Ar], the images of these two maps intersect only at finitely many points.

As remarked above, this last fact implies (with [Fe, 2.10.27]) that there is a full measure subset $U^{\prime} \subset U$ of points $\eta$ for which $\sigma_{\eta}\left(D^{\prime}\right) \cap C$ is discrete.

(c) Lipschitz. The next step in Proposition 6.1's proof argues that the map $\Phi$ in Lemma 6.3 is Lipschitz almost everywhere. However, to make the precise statement, it is necessary to digress for the introduction of the notion of a "stable direction" for a point in $C$. To begin the digression, take $x \in C$ and introduce the map $\Theta_{0}$ of Lemma 6.6. According to Lemma 6.5, there exists $\rho>0$ and a full measure subset $\Omega \subset \mathbb{C P}^{1}$ with the property that when $\kappa \in \Omega$, then $\Theta_{0}\left(\left.E_{\rho}\right|_{\kappa}\right)$ intersects $C$ near $x$ in a finite set.

Lemma 6.7. The set $\Omega$ as defined above is open.

Proof of Lemma 6.7. If $\kappa \in \Omega$, there exists $\rho_{1}>0$ such that the intersection of $C$ with $\Theta_{0}\left(\left.E_{\rho_{1}}\right|_{\kappa}\right)$ consists solely of $x$. Since $C$ is closed, there exists $\epsilon_{1}>$ 0 with the property that the intersection of $C$ with $\Theta_{0}\left(\left.E_{\rho_{1}}\right|_{\kappa^{\prime}}\right)$ is contained in $\Theta_{0}\left(E_{\rho_{1} / 2} \mid \kappa^{\prime}\right)$ when $\operatorname{dist}\left(\kappa^{\prime}, \kappa\right)<\epsilon_{1}$. Indeed, given $\delta>0$, there exists $\epsilon>0$ such that $\operatorname{diam}\left(C \cap \Theta_{0}\left(\left.E_{\rho_{1}}\right|_{\kappa^{\prime}}\right)\right)<\delta$ when $\operatorname{dist}\left(\kappa^{\prime}, \kappa\right)<\epsilon$. Thus, one can argue as in the proof of Lemma 6.4 to find an open neighborhood of $\kappa$ in $\mathbb{C P}^{1}$ where $C \cap \Theta_{0}\left(\left.E_{\rho_{1}}\right|_{\kappa^{\prime}}\right)$ is discrete.

With $\Omega$ as above, (6.1)(4) and (6.1)(5) assign to each $\kappa \in \Omega$ a positive integer, $n_{\kappa}(x)$, which is the value of $I(\cdot)$ on the restriction of $\Theta_{0}$ to $\left.E_{\rho}\right|_{\kappa}$ for all sufficiently small $\rho>0$. It then follows from Lemma 6.7 that

$$
n_{\kappa^{\prime}}(x) \leq n_{\kappa}(x)
$$

if $\kappa^{\prime}$ is sufficiently close to $\kappa$. With the preceding understood, say that $\kappa$ is a stable direction for $x$ if $\kappa \in \Omega$ and if $n_{\kappa^{\prime}}(x)=n_{\kappa}(x)$ for all $\kappa^{\prime}$ in some neighborhood of $\kappa$. Let $\underline{\Omega} \subset \Omega$ denote the set of stable directions. By definition, $\underline{\Omega} \subset \Omega$ is open. Thus, $\Omega \subset \mathbb{C P}^{1}$ is open. This set $\Omega$ is also dense in $\mathbb{C P}^{1}$. This follows from (6.11) and the fact that there is a finite set of positive integers less than any given $n_{\kappa}(x)$. (By the way, let $n(x)$ denote the infimum of $\left\{n_{\kappa}(x): \kappa \in \Omega\right\}$. This is an infimum over a set of positive integers, so it is achieved by some $\kappa \in \Omega$. Such a $\kappa$ is a stable direction for $x$.)

End the digression. Here is a Lipschitz assertion for the map $\Phi$ in Lemma 6.3:

Lemma 6.8. Let $x \in C$ and let $\left(w_{0}, w_{1}\right)$ be the usual complex, Gaussian coordinates at $x$ but with the constraint that $\kappa_{0} \equiv[1,0] \in \mathbb{C P}^{1}$ is a stable direction. Write $C$ near $x$ as in (6.2). Then, there exists $c \geq 0$ such that

$$
\sup _{\zeta \in \Phi(0)}\{\operatorname{dist}(\zeta, \Phi(w))\} \leq c \cdot|w| .
$$


Proof of Lemma 6.8. As $\kappa_{0} \in \Omega$, there exists $\rho_{1}>0$ such that the intersection of $C$ with $\Theta_{0}\left(\left.E_{\rho_{1}}\right|_{\kappa_{0}}\right)$ occurs only at $x$. And, there exists $\epsilon_{1}>0$ such that if $\operatorname{dist}\left(\kappa_{0}, \kappa\right)<$ $\epsilon_{1}$, then the intersection of $C$ with $\Theta_{0}\left(\left.E_{\rho_{1}}\right|_{\kappa}\right)$ is contained in $\Theta_{0}\left(\left.E_{\rho_{1} / 2}\right|_{\kappa}\right)$. Thus, for such $\kappa, I\left(\Theta_{0}\left(\left.E_{\rho_{1}}\right|_{\kappa}\right)\right)$ is well defined and equals $I\left(\Theta_{0}\left(\left.E_{\rho_{1}}\right|_{\kappa_{0}}\right)\right)$ which is $n_{\kappa_{0}}(x)$. However, as $\kappa_{0}$ is stable, there exists $\epsilon_{2} \in\left(0, \epsilon_{1}\right)$ such that if $\operatorname{dist}\left(\kappa_{0}, \kappa\right)<\epsilon_{2}$, then $n_{\kappa}(x)=n_{\kappa_{0}}(x)$ also. Since $I\left(\Theta_{0}\left(\left.E_{\rho_{1}}\right|_{\kappa}\right)\right) \geq n_{\kappa}(x)$ (because of $(6.1)(4)$ and (6.1)(5)), one is forced to conclude that $C \cap \Theta_{0}\left(\left.E_{\rho_{1}}\right|_{\kappa}\right)=x$ when $\operatorname{dist}\left(\kappa, \kappa_{0}\right)<\epsilon_{2}$. This implies (6.12) where $c=\mathcal{O}\left(\rho_{1} / \epsilon_{2}\right)$.

Remark that this lemma asserts that $\Phi$ is Lipschitz at the point $x$, but it does not say anything about nearby points. To consider this issue, introduce the notion of "multiplicity" of a point in $C$. Here, the multiplicity, $n(x)$, of $x \in C$ is defined to be the infimum of the positive integers $n_{\kappa}(x)$ as $\kappa$ varies over the set $\Omega$. With the preceding understood, say that a point $x \in C$ is regular when $n(x) \leq n\left(x^{\prime}\right)$ for all $x^{\prime}$ in some open neighborhood in $C$ of $x$. (For example, $x$ is stable when $n(x)=1$.)

Lemma 6.9. The set of regular points in $C$ is open and dense. Furthermore, if $x$ is a regular point of $C$, then there is a complex Gaussian coordinate system centered at $x$ and $a$ constant $c$ with the following properties:

(1) $C$ near $X$ has the form of (6.2).

(2) The map $\Phi$ in (6.2) maps a neighborhood $D^{\prime} \subset D$ of 0 into the stratum of the space of unordered $m$-tuples where all $m$ points coincide.

(3) The map $\Phi$ is Lipschitz on $D^{\prime}$ in the sense that

$$
\left|\Phi(w)-\Phi\left(w^{\prime}\right)\right|<c\left|w-w^{\prime}\right|
$$

for all pairs $w, w^{\prime} \in D^{\prime}$.

The following is an immediate corollary of the preceding lemma:

Lemma 6.10. There is an open, dense subset of $C$ which has the structure of a Lipschitz submanifold of $X$.

(There is a notion of a 2-dimensional, rectifiable set; this being a set with finite 2dimensional Hausdorff measure which is the union of a countable number of disjoint Lipschitz submanifolds with a set having zero 2-dimensional Hausdorff measure. (See, e.g., [Fe] or [La].) Lemma 6.10 implies that $C$ is rectifiable.)

The proof of this lemma is similar in most respects to that of Lemma 6.8, so the discussion will be brief. The remainder of this subsection is occupied with this discussion.

Proof of Lemma 6.9. There are six steps to the proof.

Step 1. This step constitutes another digression to consider a family of pseudoholomorphic disks which contains Lemma 5.4's family $\left\{\Theta\left(D_{w}\right)\right\}$ and Lemma 6.6's family $\left\{\Theta_{0}\left(\left.E_{\rho}\right|_{\kappa}\right)\right\}$ as subfamilies. For this purpose, introduce $\pi: F_{U} \rightarrow X$, the principal $\mathrm{U}(2)$ reduction of the principal $\mathrm{SO}(4)$ bundle of orthonormal frames in $T X$. This principal bundle parameterizes the complex Gaussian coordinate systems centered at points in $X$. 
Given $\rho>0$, let $D \subset \mathbb{C}$ denote the radius $\rho$ disk with center 0 . Lemma 5.5 gives $\rho>0$ and a smooth map $\underline{\Theta}: D \times D \times F_{U} \rightarrow X$ with the following properties:

(1) $\underline{\Theta}(\cdot, \cdot, u)$ are Lemma 5.4's maps $\Theta$ as defined using the complex

Gaussian coordinate system which is defined by $u$ (and so centered at $x=\pi(u))$.

(2) $\underline{\Theta}\left(D_{0}, 0, u\right)=\Theta_{0}\left(\left.E_{\rho}\right|_{\kappa}\right)$, where $\Theta_{0}$ is from Lemma 6.6 as applied to the point $\pi(u)$ and where $\kappa$ is the complex line given by $w_{1}=0$ in the complex Gaussian coordinates defined by $u$.

Remark that if $x \in X$ is fixed, with a trivialization of $F_{U}$ on a ball $B \subset X$ centered at $x$, then $\underline{\Theta}$ can be restricted to define a smooth map (also called $\underline{\Theta}$ ) from $D \times D \times \mathrm{U}(2) \times B$ into a neighborhood of $x$ in $X$. Furthermore, if $\kappa \in \mathbb{C P}^{1}$ is fixed, then the fibration $\mathrm{U}(2) \rightarrow \mathbb{C P}^{1}$ has a section, $s$, on a neighborhood $U_{\kappa} \subset \mathbb{C P}^{1}$ of $\kappa$. Using this section, the map $\underline{\Theta}$ defines a smooth map,

$$
\underline{\Theta}_{1}: D \times D \times U_{\kappa} \times B \rightarrow X .
$$

Step 2. This step is a preliminary one to consider properties of $\underline{\Theta}_{1}$ with respect to $C$. So, suppose that $x \in C$ and that $\underline{\Theta}_{1}$ is defined near $x$. First of all, there is a direction $\kappa_{0} \in \mathbb{C P}^{1}$ which is a stable direction for $x$ and is such that $n_{\kappa_{0}}(x)=n(x)$. Since $\kappa_{0}$ is a stable direction for $x$, the intersection of $C$ with $\underline{\Theta}_{1}\left(\cdot, 0, \kappa_{0}, x\right)$ is a finite set, and by choosing $\rho$ smaller if necessary, this set can be assumed to be $\{x\}$. The stability of $\kappa_{0}$ for $x$ also implies that the intersection $\underline{\Theta}_{1}(\cdot, 0, \kappa, x) \cap C=\{x\}$ for all $\kappa$ in some open neighborhood in $\mathbb{C P}^{1}$ of $\kappa_{0}$.

If $\kappa$ is close to $\kappa_{0}$, and $w$ is close to 0 , and $x^{\prime}$ is close to $x$, then $\underline{\Theta}_{1}\left(\cdot, w, \kappa, x^{\prime}\right)^{-1}(C)$ is a finite set of points, and this set is contained in the concentric subdisk in $D$ of radius $\rho / 2$. (This is proved by repeating, virtually verbatim, the proof of Lemma 6.4.) In fact, given $\delta>0$, one can be assured that $\underline{\Theta}_{1}\left(\cdot, w, \kappa, x^{\prime}\right)^{-1}(C)$ is contained in the radius $\delta$ disk about 0 when $w$ is sufficiently close to $0, \kappa$ sufficiently close to $\kappa_{0}$, and $x^{\prime}$ sufficiently close to $x$. With the preceding understood, it follows that $I\left(\underline{\Theta}_{1}\left(\cdot, w, \kappa, x^{\prime}\right)\right)$ is well defined and is equal to $n(x)$ if $\kappa$ is close to $\kappa$, and $w$ is close to 0 , and $x^{\prime}$ is close to $x$. This last fact plus (6.1)(4) and (6.1)(5) together imply that $\underline{\Theta}_{1}\left(\cdot, w, \kappa, x^{\prime}\right)^{-1}(C)$ contains no more than $n(x)$ points if $\kappa$ is close to $\kappa_{0}$, and $w$ is close to 0 , and $x^{\prime}$ is close to $x$.

Step 3. Suppose that $x \in C$ is regular. This step proves that $\underline{\Theta}_{1}\left(\cdot, w, \kappa, x^{\prime}\right)^{-1}(C)$ contains precisely one point if $w$ is near 0 , and $\kappa$ is near $\kappa_{0}$, and $x^{\prime}$ is close to $x$. This follows from the assumed regularity of $x$. Indeed, if this set contains two points, say $\left\{x_{1}, x_{2}\right\}$, then both will be close to $x$. And, there is some $\kappa^{\prime} \in \mathbb{C P}^{1}$ which is close to $\kappa$ such that $x_{2} \in \underline{\Theta}_{1}\left(\cdot, 0, \kappa^{\prime}, x_{1}\right)$. (Argue here as in the proof of Lemma 6.8 , and with the help of (6.13).) The point is, the image disk of $\underline{\Theta}_{1}\left(\cdot, 0, \kappa^{\prime}, x_{1}\right)$ contains two points of $C$. This is compatible with its $I$ value being $n(x)$ only if $n\left(x_{1}\right)<n(x)$. The latter contradicts the assumed regularity of $x$.

Step 4. Suppose $x \in C$ is regular. Then, the fact that the set $\underline{\Theta}(\cdot, w, \kappa, x)^{-1}(C)$ is a single point when $w$ is near 0 and $\kappa$ is near $\kappa_{0}$ implies that the map $\Phi$ from Lemma 6.3 (as defined using a complex Gaussian coordinate system which is compatible with $\left.\kappa_{0}\right)$ is Lipschitz near the origin. The proof mimics the proof of Lemma 6.8 (using (6.13) and (6.14) to generalize Lemma 6.6) and is omitted. 
Step 5. This step establishes that the set of regular points in $C$ is open. Indeed, this follows from the claim that if $x$ is regular, then $n(x)=n\left(x^{\prime}\right)$ when $x^{\prime}$ is sufficiently close to $x$. The latter follows directly from the results of Steps 2 and 3.

Step 6. This step proves that the regular points are dense. Indeed, let $x \in C$ and let $V \subset C$ be any open neighborhood of $x$. Consider the function $n(\cdot)$ on $V$. This is a positive integer valued function, so it must achieve its minimum. Such a minimizer is regular.

(d) $C$ is pseudo-holomorphic near regular points. It has now been established that the set $C$ has the structure of a Lipschitz submanifold of $X$ at the open and dense set of regular points. This subsection establishes

Lemma 6.11. Let $x \in C$ be a regular point. There is a neighborhood of $x$ in $C$ which is an embedded, pseudo-holomorphic disk.

The remainder of this subsection is occupied with the

Proof of Lemma 6.11. The proof requires six steps.

Step 1. Fix a regular point $x \in C$. Lemma 6.9 provides complex Gaussian coordinates centered at $x$ with the following properties: Introduce Lemma 5.4's map $\Theta$ as defined by a complex Gaussian coordinate system centered at $x$. This coordinate system should be chosen so that the complex line $\kappa_{0}$ where $w_{1} \equiv 0$ obeys $n_{\kappa_{0}}(x)=n(x)$. With $\Theta$ understood, note that $\Theta^{-1}(C)$ has the form $(\Phi(w), w)$ when $w$ is in some open neighborhood $D^{\prime} \subset D$ of the origin. Here, $\Phi: D^{\prime} \rightarrow D$ is a Lipschitz map sending 0 to 0 .

There is some simplification of notation when $C$ is considered in the complex Gaussian coordinate system instead of the image of this coordinate system by $\Theta$. With this purpose in mind, note that because $\Phi$ is Lipschitz, there exists a disk $D^{\prime \prime} \subset D^{\prime}$ centered at 0 with the property that when $w \in D^{\prime \prime}$, then each $D_{w}$ intersects $C$ in a single point $\Phi_{1}(w)$. And, $\Phi_{1}(\cdot)$ defines a complex valued, Lipschitz map from $D^{\prime \prime}$ to $D$.

Step 2. Lemma 6.11 is proved by demonstrating that $\Phi_{1}$ is holomorphic at $x=0$. One can then let $x$ vary to conclude that $C$ is an embedded, pseudo-holomorphic submanifold at every stable point.

To prove that $\Phi_{1}$ is holomorphic at 0 , introduce the complex valued function $\partial \Phi_{1}: D^{\prime \prime}-0 \rightarrow \mathbb{C}$ by the rule

$$
\partial \Phi_{1}(w) \equiv \frac{\Phi_{1}(w)}{w} .
$$

Since $\Phi_{1}$ is Lipschitz, this map is bounded on $D^{\prime \prime}-0$. The point is that $\Phi_{1}$ is holomorphic at 0 if and only if $\partial \Phi_{1}$ has a continuous extension to $D^{\prime \prime}$.

With the preceding understood, suppose that $\partial \Phi_{1}$ does not extend continuously over to the whole of $D^{\prime}$. This supposition is shown below to contradict the assumptions in (6.1). Indeed, the assumption that $\partial \Phi_{1}$ is not continuous at 0 will allow 
for a map of a disk in $X$ which has the following properties:

(1) $\sigma$ is admissible.

(2) $\sigma$ is homotopic (via a homotopy where the inverse image of $C$ is compact) to the image of the $\sigma_{0}$ which sends $\zeta$ to $\Theta\left(\zeta^{2}, 0\right)$.

(3) $I(\sigma)>I\left(\sigma_{0}\right)$.

Such a map $\sigma$ is prohibited by (6.1) thus voiding the assumption that $\partial \Phi_{1}$ has no continuous extension over $D^{\prime}$. (Note that $\sigma_{0}$ is not, generically, 1-1.)

Step 3. To construct the map $\sigma$, consider that if $\partial \Phi_{1}$ is not continuous at 0 , then there are sequences $\left\{a_{1 i}\right\}$ and $\left\{a_{2 i}\right\}$ in $D^{\prime}-0$ which converge to 0 with the following properties:

$$
\begin{aligned}
& \text { (1) } \lim _{i \rightarrow \infty} \partial \Phi_{1}\left(a_{1 i}\right) \equiv c_{1 \infty} \text { exists. } \\
& \text { (2) } \lim _{i \rightarrow \infty} \partial \Phi_{1}\left(a_{2 i}\right) \equiv c_{2} \text { exists. } \\
& \text { (3) } c_{1 \infty} \neq c_{2^{\infty}} . \\
& \text { (4) }\left|c_{2 \infty}\right| \geq\left|c_{1 \infty}\right| .
\end{aligned}
$$

There is no generality lost in assuming that

$$
2 \delta>\left|\partial \Phi_{1}\left(a_{1 i}\right)-\partial \Phi_{1}\left(a_{2 j}\right)\right|>\delta
$$

for some $\delta>0$ and all pairs $(i, j)$.

With the preceding understood, choose $i$ large and set $a_{1} \equiv a_{1 i}$ and $c_{1} \equiv$ $\partial \Phi_{1}\left(a_{1 i}\right)$. Choose $j \gg i$ so that $a_{2} \equiv a_{2 j}$ obeys

$$
\left|a_{2}\right| \ll\left|a_{1}\right| .
$$

Set $c_{2} \equiv \partial \Phi_{1}\left(a_{2 j}\right)$.

Consider the map $\sigma_{1}: \mathbb{C} \rightarrow \mathbb{C}^{2}$ which sends $\zeta$ to $\left(w_{0}(\zeta), w_{1}(\zeta)\right)$, where

$$
\text { (1) } \begin{aligned}
w_{0}(\zeta) \equiv & \left(\frac{c_{1}-c_{2}}{a_{1}-a_{2}}+\eta_{2}\right) \cdot \zeta^{2}+\left(\frac{c_{2} a_{1}-c_{1} a_{2}}{a_{1}-a_{2}}+\eta_{1}\right) \cdot \zeta+\eta_{0} \\
& +\eta \cdot \zeta\left(\zeta-a_{1}\right)\left(\zeta-a_{2}\right),
\end{aligned}
$$

$$
\text { (2) } w_{1}(\zeta) \equiv \zeta .
$$

Here, $\eta$ and $\eta_{0,1,2}$ are small constants which will be chosen shortly. (Note that when $\eta_{i}$ are all zero, then the image of $\sigma_{1}$ intersects $C$ at three or more points as $\sigma_{1}$ 's image hits $(0,0)$ and $\left(\Phi_{1}\left(a_{1}\right), a_{1}\right)$ and $\left(\Phi_{1}\left(a_{2}\right), a_{2}\right)$. This is the point of choosing $\sigma_{1}$ in this way. The map $\sigma_{1}$ will shortly be perturbed to be pseudo-holomorphic, and then the $\eta$ 's will be chosen to insure that the resulting map has finitely many, but at least three intersections with $C$.)

Step 4. As remarked above, $\sigma_{1}$ is not pseudo-holomorphic. Consider perturbing this map to a pseudo-holomorphic map. To be precise, the plan is to find a pseudoholomorphic $\sigma$ which sends $\zeta$ to

$$
\sigma(\zeta) \equiv\left(w_{0}(\zeta)+\tau, \zeta\right),
$$

where $\tau$ is a smooth function on the complex plane which obeys (5.11) using $f \equiv$ $w_{0}(\zeta)$ and using

$$
\rho \equiv \frac{R\left|a_{1}\right|\left(\left|c_{2}\right|+1\right)}{\delta}
$$


for a fixed $R \geq 100$. In particular, choose $R$ to be 100 or more times the Lipschitz constant of $\Phi$ at 0 . In considering (5.11), take each

$$
\begin{aligned}
& \text { (1) }\left|\eta_{i}\right|<s_{0} \cdot \rho^{2-i} . \\
& \text { (2) }|\eta|<\rho^{4} .
\end{aligned}
$$

Here $s_{0}$ is a constant which depends on the choice of $R$ and on the parameters $\delta$ and $\left|c_{2}\right|$ but not on $\left\{a_{1}, a_{2}\right\}$.

The function $\tau$ is found with the help of Lemma 5.5. In this regard, remember that $w_{0}(\zeta)$ plays the role of the polynomial $f$; and with this understood, note that

$$
\left\|\chi_{\rho} w_{0}\right\| \leq s_{1} \cdot \rho
$$

when (6.23) is satisfied. Here, $s_{1}$ depends on the parameters $R, \delta$ and $\left|c_{2}\right|$, but not on $\left\{a_{1}, a_{2}\right\}$.

Remember that $R$ and $\left|c_{2}\right|$ and $\delta^{-1}$ are uniformly bounded, while $\left|a_{1}\right|$ can be taken as small as desired. Thus, Lemma 5.5 can be invoked when $\left|a_{1}\right|$ is sufficiently small, less than some $s_{2}>0$ which is provided by Lemma 5.5 and the values of $R, \delta$ and $\left|c_{2}\right|$. Thus, with $\left|a_{1}\right|$ small, Lemma 5.5 describes a solution $\tau$ to the $f \equiv w_{0}(\zeta)$ version of (5.11).

Step 5 . The function $\tau$ is implicitly a function of the parameters $\eta$ and $\eta_{0,1,2}$. These parameters will now be fixed by the following requirements:

$$
\begin{aligned}
& \text { (1) } \sigma^{-1}(C) \text { is a discrete set. } \\
& \text { (2) } \sigma(D) \cap C \text { contains } x \text { and } \Theta\left(\Phi\left(a_{1}\right), a_{1}\right) \text { and } \Theta\left(\Phi\left(a_{2}\right), a_{2}\right) \text {. }
\end{aligned}
$$

Here is the strategy for solving the preceding constraints: For fixed but small $\eta$ (as in $(6.23))$, solve the following equations for $\left(\eta_{0}, \eta_{1}, \eta_{2}\right)$ :

$$
\begin{aligned}
& \text { (1) } \eta_{2} a_{1}^{2}+\eta_{1} a_{1}+\eta_{0}+\tau\left(a_{1}\right)=0 \\
& \text { (2) } \eta_{2} a_{2}^{2}+\eta_{1} a_{2}+\eta_{0}+\tau\left(a_{2}\right)=0 \\
& \text { (3) } \eta_{0}+\tau(0)=0
\end{aligned}
$$

If these constraints are satisfied, then so is $(6.25)(2)$. It is an exercise with the implicit function theorem and Lemma 5.5 to prove that (6.26) has a unique solution obeying (6.25)(1) when $\rho$ is small. This solution will then be an implicit function of the parameter $\eta$. With this understood, consider the map, $h$, from the radius $\rho^{3}$ disk in $\mathbb{C}$ to $\mathbb{C}$ which sends $\eta$ to value of $w_{0}(\zeta)+\tau(\zeta)$ at a point $\zeta \equiv \frac{a_{1}}{2}$. It is a second exercise with Lemma 5.5 and the implicit function theorem to prove that the differential of $h$ at $\eta=0$ is surjective. This implies (with Aronszajn's unique continuation theorem $[\mathrm{Ar}]$ ) that the images of the maps $\sigma$ for any two different values of $\eta$ (near 0 ) intersect in a discrete set. One can conclude from this last fact and $[\mathrm{Fe}, 2.10 .27]$ that there is a full measure set of $\eta$ 's $($ near $\eta=0)$ for which the map $\sigma$ intersects the curve $C$ in a discrete set.

Step 6 . Choose $\eta$ now so that $(6.23)(2)$ is obeyed and so that $\sigma^{-1}(C)$ is discrete. The next task is to compute the value of $I(\sigma)$. As indicated at the outset, there are two different ways to compute this number. For the first, remark that when $|\zeta| \geq \frac{3 \rho}{4}$, then

$$
\left|w_{0}+\tau\right|>\frac{R}{2}|\zeta|
$$


Since $R$ is chosen to be at least 100 times the Lipschitz constant of $\Phi$, one can conclude that the map $\sigma$ is admissible and that $\sigma$ is homotopic as described in $(6.16)(2)$ to the map $\sigma_{0}$ which sends $\zeta$ to $\Theta\left(\zeta^{2}, 0\right)$. This implies (with (6.1)(3)) that $I\left(\sigma_{0}\right)=2 \cdot n(x)$.

On the other hand, the map $\sigma$ is a pseudo-holomorphic embedding of a disk which intersects $C$ in a finite set of points. Each such point contributes a positive integer to $I(\sigma)$ because of $(6.1)(4)$ and $(6.1)(5)$. In particular, $\sigma^{-1}(C)$ contains at least 3 points. Thus, for example, if $n(x)=1$, then $I(\sigma) \geq 3$ which is greater than the value 2 computed in the previous paragraph. In the case where $n(x)>1$, then $I(\sigma) \geq 3 \cdot n(x)$ and here is why:

Lemma 6.12. Let $x \in C$, and let $\sigma: D \rightarrow X$ be a pseudo-holomorphic embedding of a disk whose image intersects $C$ only at $x$. Then $I(\sigma) \geq n(x)$.

The proof below of Lemma 6.12 completes the proof of Lemma 6.11.

Proof of Lemma 6.12. Without loss of generality, suppose that $\sigma^{-1}(x)=0$. Because of $(6.1)(4), I(\sigma)=I\left(\sigma^{\prime}\right)$, where $\sigma^{\prime}$ is the restriction of $\sigma$ to any subdisk $D^{\prime} \subset D$ which contains the origin. Remember this fact.

The simplest case occurs when the tangent plane $\kappa$ to the image of $\sigma$ at $x$ is a stable direction. In this case Lemma 6.8 and (6.1)(2) imply that the contribution to $I(\sigma)$ from $x$ is the same as $n_{\kappa}(x)$, which is at least as big as $n(x)$. Indeed, because of Lemma 6.8, the restriction of $\sigma$ to a very small radius disk about 0 (say of radius $\rho>0)$ will be homotopic to $\Theta_{0}\left(\left.E_{\rho}\right|_{\kappa}\right)$ via a homotopy with compact $C$-inverse.

When $\kappa$ is not a stable direction, restrict anyway to a very small radius disk $D^{\prime} \subset D$ which contains the origin. One can suppose that $\left.\sigma\right|_{D^{\prime}}$ is given in some complex Gaussian coordinate system centered at $x$ by (5.10) with $f \equiv \tau \equiv 0$ and with $u$ obeying $u(0)=0$ and $\left.d u\right|_{0}=0$. With this understood, consider perturbing $\left.\sigma\right|_{D^{\prime}}$ by adding $f(\zeta) \equiv \eta_{0}+\eta_{1} \cdot \zeta$ and Lemma 5.7's $\tau$ to (5.10). Here, $\eta_{0}$ and $\eta_{1}$ should be taken very small. Furthermore, with $\eta_{1}$ chosen, one can use the implicit function theorem (with the help of Lemma 5.5) to choose $\eta_{0}$ as a smooth function of $\eta_{1}$ so that the perturbed map, $q$, obeys $q(0)=0$. Since $\eta_{0}$ is now a function of $\eta_{1}$, the assignment to $\eta_{1}$ of the $\left.\frac{\partial(f+\tau)}{\partial \zeta}\right|_{\zeta=0}$ defines a smooth map from a small disk about 0 in $\mathbb{C}$ to $\mathbb{C}$. The estimates in Lemma 5.5 show that the differential of this map at $\eta_{1}=0$ is surjective. Thus, there are arbitrarily small choices for $\eta_{1}$ which make the tangent plane $\kappa^{\prime}$ to $q$ at $x$ a stable direction.

With the preceding understood, remark that because $C$ is closed and only intersects $\sigma\left(D^{\prime}\right)$ at $x$, the map $q$ will be admissible for all sufficiently small $\left|\eta_{1}\right|$. And, for sufficiently small $\left|\eta_{1}\right|$, the map $q$ will be homotopic to $\left.\sigma\right|_{D^{\prime}}$ by a homotopy with compact $C$-inverse image. This implies $I(q)=I(\sigma)$. However, $q\left(D^{\prime}\right) \cap C$ contains at least the point $x$. And one can argue (as in the proof of Lemma 6.4) that $q\left(D^{\prime}\right) \cap C$ is a finite set. Thus, $I(q)$ is at least as big as the $I$ value of $q$ 's restriction to some smaller radius disk $D^{\prime \prime} \subset D^{\prime}$ whose $q$ image intersects $C$ only at $x$. If $\eta_{1}$ is chosen so that the tangent plane to $q$ at 0 is a stable direction, then, as noted previously, this $I$ value is at least $n(x)$. Thus $I(\sigma)=I(q) \geq n(x)$ as claimed.

(e) The singular points. A point $x \in C$ which is not regular will be called singular. Here is the first basic fact about singular points:

Lemma 6.13. There are finitely many singular points of $C$.

The remainder of this subsection is occupied with the proof of this lemma. 
Proof of Lemma 6.13. The proof requires ten steps.

Step 1. To begin, fix attention on a singular point $x \in C$. Choose a complex, Gaussian coordinate system centered at $x$ which is compatible with a stable direction $\kappa$ for which $n_{\kappa}(x)=n(x)$. Introduce the map $\Theta$ of Lemma 5.4. This map defines complex coordinates $(\zeta, u)$ on a neighborhood of $x$ in $X$. (These coordinates are generally not the complex Gaussian coordinates as the disks $u=$ constant are pseudo-holomorphic.) Note that these $(\zeta, u)$ coordinates are not uniquely defined by Lemma 5.4; and it proves convenient to exploit the freedom in choosing $(\zeta, u)$ coordinates to put the almost complex structure $J$ in a nice form.

To begin this task, consider the almost complex $J$ in any $\Theta$ coordinates $(\zeta, u)$ on $D \times D^{\prime}$. Because the disks of constant $u$ are pseudo-holomorphic, $J$ must decompose as follows with respect to the (product induced) splitting $T\left(D \times D^{\prime}\right)=T D \oplus T D^{\prime} \approx$ $\mathbb{R}^{2} \oplus \mathbb{R}^{2}$ :

$$
J=\left(\begin{array}{cc}
a & b \\
0 & a^{\prime}
\end{array}\right)
$$

where $a, a^{\prime}$ and $b$ are $2 \times 2$ matrix valued functions on $D \times D^{\prime}$ which obey

$$
\begin{aligned}
& \text { (1) } a \cdot a=a^{\prime} \cdot a^{\prime}=-\mathbb{I} \text {. } \\
& \text { (2) } a \cdot b+b \cdot a^{\prime}=0 . \\
& \text { (3) }\left.a a^{\mathrm{T}}\right|_{0}=\left.a^{\prime} a^{\prime \mathrm{T}}\right|_{0}=\mathbb{I} . \\
& \text { (4) }\left.b\right|_{0}=0 .
\end{aligned}
$$

Here, $\mathbb{I}$ is the identity matrix, and the superscript $\mathrm{T}$ denotes the transpose.

It proves useful to refine this choice of coordinates by imposing further constraints on the matrices $a, a^{\prime}$ and $b$. To begin, remark that by taking a smaller diameter $D^{\prime}$ if necessary, Lemma 5.4 provides a smooth map $\tau: D^{\prime} \rightarrow \mathbb{R}^{2}$ with the following properties: First, $\tau(0)=0$. Second, the embedding of $D^{\prime}$ into $D \times D^{\prime}$ which sends $u$ to $(\tau(u), u)$ is pseudo-holomorphic. With the preceding understood, change to the coordinates $\left(\zeta_{1} \equiv \zeta+\tau(u), u_{1} \equiv u\right)$. With these coordinates, the $u_{1}=$ constant disks are pseudo-holomorphic as is the disk $\zeta_{1}=0$. Thus, in the $\left(\zeta_{1}, u_{1}\right)$ coordinates, $J$ has the same form as in (6.28)-(6.29) and the matrix $b$ obeys

$$
b(0, \cdot) \equiv 0 .
$$

A further change to $\left(\zeta_{2} \equiv g\left(\zeta_{1}, u\right) \cdot \zeta_{1}, u_{2} \equiv g^{\prime}\left(u_{1}\right)\right.$ ) (for suitable smooth matrix valued function $g$ and map $g^{\prime}$ ) can be made so that $J$ in (6.28) obeys (6.29) and (6.30) and also

$$
a \equiv\left(\begin{array}{cc}
0 & -1 \\
1 & 0
\end{array}\right) \quad \text { and } \quad a^{\prime}(0, \cdot) \equiv\left(\begin{array}{cc}
0 & -1 \\
1 & 0
\end{array}\right) .
$$

With the preceding understood, henceforth use $(\zeta, u)$ to denote $\left(\zeta_{2}, u_{2}\right)$. That is, the $(\zeta, u)$ coordinates will always be chosen so that $J$ obeys (6.29)-(6.31).

Step 2. Note that in the $\Theta$ coordinates $(\zeta, u)$ above, the set $C$ has the form in (6.2) where $\Phi$ is a continuous map from $D^{\prime}$ into the space, $\mathbb{C}_{n}$, of $n$-tuples of points in $\mathbb{C}$. Here $n \equiv n(x)$. This step serves as a digression to describe this space. To begin, observe that there are two natural analytic structures on $\mathbb{C}_{n}$. For the first, $\mathbb{C}_{n}$ has the structure of a stratified space, where a typical stratum is labeled by an $n$-tuple $L \equiv\left(n_{1}, \ldots, n_{n}\right)$ of non-negative integers subject to the constraint $\sum_{j} j \cdot n_{j}=n$. If $L$ is such an $n$-tuple, use $\mathbb{C}_{n, L}$ to denote the corresponding 
stratum. Here, $\mathbb{C}_{n, L}$ corresponds to those $n$-tuples where there are $n_{1}$ distinct points, $n_{2}$ coinciding pairs, $n_{3}$ coinciding triples, etc. The top dimensional stratum is labeled by $L_{0} \equiv(n, 0, \ldots, 0)$. The lowest dimensional stratum is labeled by $(0,0, \ldots, 1)$.

The stratum $\mathbb{C}_{n, L_{0}}$ is naturally diffeomorphic to $\left(\times_{n} \mathbb{C}-\Delta\right) / S_{n}$, where $\Delta$ is the union of the subsets of ordered $n$-tuples with coinciding points, and where $S_{n}$ is the symmetric group on $n$ letters. In general each stratum is a smooth manifold; here

$$
\mathbb{C}_{n, L} \approx \times_{j}\left(\mathbb{C}_{n j, L_{0}}\right) \text {. }
$$

(Set $\mathbb{C}_{0, L_{0}} \equiv$ point.)

There is a partial ordering to the strata which is defined as follows: Say that $L \leq L^{\prime}$ if $L$ is in the closure of $L^{\prime}$. For this condition to hold, the corresponding $n$-tuples of $L$ and $L^{\prime}$ must obey

$$
\sum_{j \geq k} j \cdot n_{j} \geq \sum_{j \geq k} j \cdot n_{j}^{\prime} \quad \text { for all } k \in\{1,2, \ldots, n\} .
$$

Note that the codimension of the strata $L$ is equal to $2 \cdot\left(n-\sum_{j} n_{j}\right)$.

The second picture of $\mathbb{C}_{n}$ comes by thinking of $n$-complex numbers as the roots of an $n$ 'th order polynomial

$$
\zeta^{n}+v_{1} \zeta^{n-1}+\cdots+v_{n}
$$

The coefficients $\left(v_{1}, \ldots, v_{n}\right)$ identify $\mathbb{C}_{n}$ with $\mathbb{C}^{n}$.

Note that if $t \equiv\left(\zeta_{1}, \ldots, \zeta_{n}\right)$ is an $n$-tuple in $\mathbb{C}_{n}$, then

$$
v_{j}(t)=\sum_{\sigma} \zeta_{\sigma_{1}} \cdots \zeta_{\sigma_{j}}
$$

where the sum is over all unordered subsets $\sigma$ of $j$ distinct elements in $\{1, \ldots, n\}$. In any event, (6.35) defines a standard, 1-1 onto map of $\mathbb{C}_{n}$ onto $\mathbb{C}^{n}$. Note, as an aside, that the tautological map from $\left(\times_{n} \mathbb{C}\right)-\Delta$ into $\mathbb{C}^{n}$ is a smooth covering map. Likewise, if $L$ is any stratum, then the tautological map from $\times_{j}\left(\left(\times_{n j} \mathbb{C}\right)-\Delta\right)$ into $\mathbb{C}_{n, L}$ is also a smooth covering map.

In the $\mathbb{C}^{n}$ picture, each stratum $\mathbb{C}_{n, L}$ of $\mathbb{C}_{n}$ corresponds to a Zariski open subset of some algebraic subset (defined by polynomial equations) of $\mathbb{C}^{n}$. It is important to note that in terms of the variables $\left(\zeta_{1}, \ldots, \zeta_{n}\right)$ for $\mathbb{C}_{n}$, these polynomials are homogeneous and symmetric, but they are generally neither in terms of the variables $\left(v_{1}, \ldots, v_{n}\right)$ for $\mathbb{C}^{n}$. For example, in the variables $\left(\zeta_{1}, \ldots, \zeta_{n}\right), \mathbb{C}_{n, L_{0}}$ is the complement in $\mathbb{C}^{n}$ of the zero set of the discriminant function $\prod_{i \neq j}\left(\zeta_{i}-\zeta_{j}\right)$. But, in the case where $n=2$, this polynomial is written in terms of the $v$ 's as set of the equation $v_{1}^{2}-4 v_{2}=0$.

Step 3. Let $\pi: C \rightarrow D^{\prime}$ denote the projection onto the second coordinate. (To save notation here and henceforth in this subsection, $C$ is identified with its inverse image via $\Theta$ in $D \times D^{\prime}$. Thus, $\pi^{-1}(u)=\Phi(u)$.) It has already been established that there is a dense, open subset in $C$ which has the structure of a complex manifold. With this understood, Sard's theorem gives a dense open subset $D_{0}^{\prime} \subset D$ with the following properties:

(1) $C_{0} \equiv C \cap \pi^{-1}\left(D_{0}^{\prime}\right)$ is a smooth, pseudo-holomorphic submanifold of $D \times D^{\prime}$.

(2) The projection $\pi: C_{0} \rightarrow D_{0}^{\prime}$ is a covering map. 
With the preceding understood, it follows that $\Phi$ maps $D_{0}^{\prime}$ smoothly into $\mathbb{C}^{n}$.

Step 4 . Let $f: \mathbb{C}^{n} \rightarrow \mathbb{C}$ be a polynomial in the complex coordinates which is symmetric and homogeneous as a function of the coordinates $\left(\zeta_{1}, \ldots, \zeta_{n}\right)$. This function is pulled back to $D^{\prime}$ by $\Phi$ and so gives a complex valued function on $D^{\prime}$. (Denote this pull-back function by $f$ also.) The purpose of this step is to show that $f$ 's restriction to $D_{0}^{\prime}$ obeys a variant of the Cauchy-Riemann equations.

A digression is required to derive this equation. To start the digression, fix a point $w \in D_{0}^{\prime}$. On a neighborhood $U \subset D_{0}^{\prime}$ of $w$, the map $\pi: C \rightarrow D^{\prime}$ is a trivial covering map with some number $m \leq n$ sheets. Thus, there are some $m$ smooth, complex valued functions $\left(\varphi_{1}, \ldots, \varphi_{m}\right)$ on a neighborhood of $U \subset D_{0}^{\prime}$ of $w$ such that the $m$ sheets of $C$ over $U$ are the graphs of these functions. (Needless to say, each $\varphi_{j}$ maps into $D$.) It proves convenient to take $m=n$, and for this purpose, remark that each sheet of $C$ over $U$ has an associated positive integer multiplicity, this being the value of $n\left(x^{\prime}\right)$ for any $x^{\prime}$ in said sheet. Note that the sum over the $m$ sheets of these multiplicities is equal to $n$. With the preceding understood, add as many copies of $\varphi_{j}$ as is the multiplicity of the $j$ th sheet and then renumber the resulting $n$ functions from 1 to $n$.

Each sheet over $U$ is a pseudo-holomorphic submanifold of $D \times D^{\prime}$. This implies that $\varphi \in\left\{\varphi_{j}\right\}_{j=1}^{n}$ obeys a differential equation which is described as follows: Think of $\varphi$ as a complex valued function on a domain in $\mathbb{R}^{2}$, and let $d \varphi$ denote its differential. Then, at a point $u \in U$, the function $\varphi$ obeys the equation

$$
d \varphi+i \cdot a^{\prime} \cdot d \varphi+\mathfrak{b}=0 .
$$

Here, $a^{\prime}$ is evaluated at the point $(\varphi(u), u)$, and $\mathfrak{b}$ is a complex valued differential whose real and imaginary parts are linear combinations of the components of the $2 \times 2$ matrix $b$ at the point $(\varphi(u), u)$.

Because of (6.29) and (6.31), this last equation is obeyed if and only if its projection onto the $(0,1)$ part of the cotangent bundle of $U$ is zero. Thus, $(6.37)$ can be replaced by an equation of the form

$$
\frac{\partial \varphi}{\partial \bar{u}}+s_{0}(\varphi(u), u) \cdot \frac{\partial \varphi}{\partial u}+s_{1}(\varphi(u), u)=0,
$$

where $s_{0}$ and $s_{1}$ are smooth functions on $D \times U$. Furthermore, both $s_{0}$ and $s_{1}$ vanish along $0 \times U$. This follows from (6.30) and (6.31).

With the preceding understood, return now to the pull-back, $f$, of a complex polynomial on $\mathbb{C}^{n}$. Assume that as a polynomial in the variables $\left(\zeta_{1}, \ldots, \zeta_{n}\right)$ for $\mathbb{C}_{n}$, this function is symmetric and homogeneous in each $\zeta_{j}$ of degree $p \geq 0$. Then, it follows from (6.38) that $f$ obeys the equation

$$
\frac{\partial f}{\partial \bar{u}}+h \cdot f=0
$$

where

$$
h \equiv p \cdot \sum_{j} \frac{1}{\varphi_{j}}\left(s_{0}\left(\varphi_{j}, \cdot\right) \cdot \frac{\partial \varphi_{j}}{\partial u}+s_{1}\left(\varphi_{j}, \cdot\right)\right) .
$$

Remark that these last equations are not local to $U$, but make sense over the whole of $D_{0}^{\prime}$.

Step 5. The investigations of $f$ require an understanding of the function $h$ in (6.40). The following lemma summarizes: 
Lemma 6.14. The function $h$ extends over $D^{\prime}$ as an $L^{2}$ function.

Proof of Lemma 6.14. First of all, both $s_{0}$ and $s_{1}$ are differentiable and $s_{0}(0, \cdot)=$ $0=s_{1}(0, \cdot)$, so the question is really about the behavior of

$$
\sum_{j}\left|d \varphi_{j}\right|^{2}
$$

To compute the integral of $(6.41)$ over $D^{\prime}$, first remember that $C$ has finite 2dimensional Hausdorff measure. This measure is the same as that of the regular points of $C$. Because the latter subset is a pseudo-holomorphic submanifold, its area is the same as the integral of the pull-back of the symplectic form. The integral of the latter over $\pi^{-1}(U)$ can be expressed as an integral over $U$. Using (6.38) (with the fact that $\omega \approx \omega_{0}$ in the $\Theta$ coordinates), one finds that the latter integral gives a uniform bound for integral over $U$ of (6.41). This implies that $h$ is in $L^{2}\left(D^{\prime}\right)$.

Step 6 . Let $D_{2} \subset D^{\prime \prime}$ denote the concentric disk whose radius is $\frac{1}{2}$ that of $D^{\prime}$. This step decomposes $f$ on $D_{2}$ as

$$
f=f_{0} \cdot \exp (v)
$$

where $f_{0}$ is a bounded, holomorphic function and where $v$ is an $L_{1}^{2}$ function.

The function $v$ is found by solving the equation $\frac{\partial v}{\partial \bar{u}}=h$. Standard estimates with the Euclidean Laplacian on $D^{\prime}$ find such a $v$ which is square integrable with square integrable first derivative. (The $L_{1}^{2}$ norm of $v$ can be assumed to be bounded by a uniform multiple of $\epsilon \cdot\|h\|_{L^{2}}$, where $\epsilon$ is the diameter of $D^{\prime}$.)

To understand $f_{0}$ in (6.42), remark that the Sobolev inequalities in dimension 2 constrain the singular behavior of an $L_{1}^{2}$ function. In particular, let $D_{1} \subset D^{\prime}$ be the concentric disk with $\frac{3}{4}$ the radius of $D^{\prime}$. Then, for any real number $\lambda, \exp (\lambda v)$ is an $L^{1}$ function on $D_{1}$ (see, e.g., [Au, Theorem 2.46].) This last fact implies that $f_{0} \equiv \exp (-v) \cdot f$ is a square integrable function on $D_{1}$ whose derivatives are integrable. Furthermore, because of (6.39), $f_{0}$ is holomorphic on the dense, open subset $D_{1} \cap D_{0}^{\prime}$. This last fact implies that $f_{0}$ is a bounded, holomorphic function on the concentric disk $D_{2} \subset D^{\prime}$.

Step 7. This step studies the zero set of $f_{0}$ and of $\exp (v)$ in the disk $D_{2}$. The case of $f_{0}$ is simple - being holomorphic, $f_{0}$ has a finite number of zeros in $D_{2}$ if it is not identically zero. As for $\exp (v)$, the claim here is that $\exp (v)$ vanishes at most on a countable set of points in $D_{2}$. (This assumes that $f_{0}$ is not identically zero.) In the last step below, $\exp (v)$ will be shown to be non-vanishing.

Remark here that there is probably an argument like that which proves Lemma 6.9 , which directly gives a pointwise bound on (6.41). The argument would presumably come by computing in two different ways the invariant $I$ for some cleverly chosen embedded, pseudo-holomorphic disks. Such a bound would imply directly that $v$ is bounded and so that $\exp (v)$ is never zero. Thus, such a bound would complete the proof of Lemma 6.13 right here and obviate the need for the remainder of the proof.

In any event, to prove the claim that $\exp (v)$ has countably many zeros, remark that $\exp (v)$ vanishes at the points where $v=-\infty$, so the issue here is the singular set of the $L_{1}^{2}$ function $v$. To study this set, first slice the closure of $D_{2}$ by the line segments with constant $\operatorname{real}(u)$. Then $v$ restricts to almost every real $(u)=$ constant line segment as an $L_{1}^{2}$ function. Second, note that $v$ is bounded on almost every $\operatorname{real}(u)=$ constant line segment. (An $L_{1}^{2}$ function on a line segment is continuous.) 
Third, since $f$ in (6.39) is continuous, the singular set of $v$ is closed. Thus, $v$ is bounded on an open, dense set of $\operatorname{real}(u)=$ constant line segments. Fourth, note that the complement of an open dense set in an interval is a countable set of points. Thus, $v$ is unbounded on at most a countable set of $\operatorname{real}(u)=$ constant line segments. Finally, by replacing the real part of $u$ by the imaginary part of $u$, one can conclude that the singular set of $v$ in $D_{2}$ consists of at most a countable subset of points.

Step 8. One can consider Steps $5-7$ for the finite set $\{f\}$ of polynomials which delineate the various strata of $\mathbb{C}_{n} \approx \mathbb{C}^{n}$. The conclusion is that each such polynomial is either identically zero on $D_{2}$ or else it vanishes on, at most, a countable set. Thus, the singular set of $C$ in $D \times D_{2}$ has, at most, countably many members. The next two steps demonstrate that these zeros are finite in number, and that they are determined by the $f_{0}$ parts of the polynomials (with respect to the decomposition in (6.42)). This is a "pull yourself up by your bootstraps" sort of argument. This step outlines the argument.

The idea is based on the fact that most members of a countable set in $\mathbb{C}$ are isolated. Thus, if one can demonstrate that a countable set has only finitely many isolated members, then that set must itself be finite. In the case at hand, all but finitely many of the zeros of the polynomials in the set $\{f\}$ are due to the zeros of the $\exp (v)$ parts in (6.42). Thus, if one can show that $v$ is bounded near the image in $D_{2}$ of an isolated singular point, then one has shown that there are at most finitely many such isolated singular points, and so finitely many singular points in all. With the preceding understood, the strategy for the required bound on $v$ is to control (6.41) by exploiting knowledge of $C$ near an isolated singular point.

Step 9 . This step uses the isolated property of a singularity of $C$ to obtain estimates for the integral of (6.41) over small diameter disks in $D_{2}$ near the singularity's image in $D_{2}$.

To begin, consider a point $u_{0} \in D_{2}$ whose inverse image via $\pi: C \rightarrow D_{2}$ contains only one singular point, $x_{0}$, of $C$. (The argument in the general case is only notationally more complicated, and is left to the reader.) Let $D_{3} \subset D_{2}$ be a small radius disk whose center is $u_{0}$ and is such that $\pi^{-1}\left(D_{3}\right)$ contains only one singular point $x_{0}$. Lemma 6.3 (and the discussion in the preceding steps) can be used to describe a neighborhood in $C$ of $x_{0}$ as follows: A neighborhood is a union of some number $m \leq n$ topologically embedded disks. Furthermore, the complement in this neighborhood of $x_{0}$ consists of disjoint, embedded, pseudo-holomorphic, punctured disks with finite energy. (Remember that $C$ has finite area.) Fix attention on one such disk $V$. The projection $\pi$ restricts to $V-x_{0}$ as an $m_{1} \leq m$ fold covering map of $D_{3}-u_{0}$. This implies that there is a diffeomorphism $\psi$, from the punctured, standard disk in $\mathbb{C}$, onto $V-x_{0}$ which composes with $\pi$ to send a point $\eta$ to $u_{0}+\delta \cdot \eta^{m_{1}}$. Here $\delta>0$ is fixed. That is,

$$
\psi(\eta)=\left(\theta(\eta), u_{0}+\delta \cdot \eta^{m_{1}}\right) .
$$

The function $\theta$ obeys an equation of the same form as in (6.38). Indeed, at each $\eta \neq 0$, the value of $\theta(\eta)$ is equal to that of $\varphi_{j}\left(u_{0}+\delta \cdot \eta^{m_{1}}\right)$ for some choice of $j$. The difference between the $\theta$ equation and (6.38) is the following. First, $s_{0}$ is multiplied by a factor of $\left(\eta^{*} / \eta\right)^{m_{1}-1}$. Second, $s_{1}$ is multiplied by a factor of $\left(\eta^{*}\right)^{m_{1}-1}$.

This equation for $\theta$ implies various a priori estimates for $|d \theta|$. Of course, $|d \theta|$ is in $L^{2}$ because the map to $D_{3}$ is conformal. However, the equation for $\theta$ can also 
be used to estimate the $L^{2}$ norm of $|d \theta|$ over a sub-disk of some radius $r<1$ in the radius $1 / 2$ disk in $\mathbb{C}$ with center 0 . Indeed, fix a point $\eta_{0}$ in this disk, and for $s>0$, let $\kappa(s)$ denote the $L^{2}$ norm of $|d \theta|^{2}$ over the disk of radius $s$ and center $\eta_{0}$. Then, integration by parts bounds $\kappa(s)$ by a sum of two terms. The first is a uniform multiple of $s^{2}$. (This comes from the $s_{1}$ term in (6.38).) The second is a uniform multiple of the integral over the boundary of the radius $s$ disk of $\left|\theta-\theta_{0}\right| \cdot|d \theta|$. Here, $\theta_{0}$ is the average value of $\theta$ on said boundary. (This second term comes by integrating by parts to relate the $L^{2}$ norm of $\frac{\partial \theta}{\partial \bar{\eta}}$ to that of $d \theta$. Note that the term with $s_{1}$ in (6.38) only affects the size of the "uniform multiple" since $s_{1}$ can be assumed much less than 1 in absolute value.) Anyway, the aforementioned boundary integral is itself bounded by a uniform multiple of the integral over the boundary of $s \cdot|d \theta|^{2}$. (Remember that the $L^{2}$-norm of a function on the circle of radius $s$ (such as $\theta-\theta_{0}$ ) whose integral is zero is bounded by $s$ times the $L_{1}^{2}$ norm of said function.)

Since the integral over the boundary circle of $|d \theta|^{2}$ is the $s$-derivative of $\kappa(s)$, one finds that $\kappa(s)$ obeys an equation of the form

$$
s \frac{d \kappa}{d s} \geq c \cdot \kappa-s^{2} .
$$

This last equation integrates to give $\kappa(s) \leq z \cdot s^{c}$ for some constants $c>0$ and $z$.

Finally remark that this last bound on $\kappa(s)$ implies a similar bound on the $L^{2}$ norm of $(6.41)$ on a radius $s$ disk in $D_{3}$. That is, the $L^{2}$ norm of $(6.41)$ on a radius $s$ disk near $u_{0}$ is bounded a priori by $z^{\prime} \cdot s^{c^{\prime}}$ for some constants $c^{\prime}>0$ and $z^{\prime}$.

Step 10. With the preceding understood, return to the function $f$ in (6.42) and in particular to the question of the vanishing of $\exp (v)$. The point is that the preceding integral estimate for the $L^{2}$ norm of $(6.41)$ on a radius $s$ disk near $s_{0}$ implies that the function $v$ is a priori bounded near $u_{0}$. (In fact, $v$ is Hölder continuous with exponent determined by $c^{\prime}$. See, e.g., [Mo, Theorem 3.5.2].) Thus, $f$ in (6.42) vanishes at $u_{0}$ only if $u_{0}$ is a zero of the holomorphic function $f_{0}$.

Apply the preceding analysis to each of the finite set of polynomials which delineate the strata of $\mathbb{C}_{n} \approx \mathbb{C}^{n}$ to learn that the zeros of these polynomials which are isolated (i.e. most of them) are zeros only of their holomorphic parts - the $f_{0}$ in (6.42). It follows that there are only finitely many isolated zeros of these polynomials, and hence only isolated zeros. This last conclusion implies Lemma 6.13.

(f) Proposition 6.1-final arguments. This section completes the proof of Proposition 6.1 with a description of the complex curve $\Sigma$ and the pseudo-holomorphic map $\varphi$.

To obtain $\Sigma$, start with the set $C_{0} \subset C$ of regular points. This set comprises an embedded, finite area, pseudo-holomorphic submanifold of $X$. And, Lemmas 6.3 and 6.13 imply that there is an open set in $C_{0}$ with compact closure (in $C_{0}$ ) whose complement is diffeomorphic to a finite, disjoint union of cylinders $[0, \infty) \times S^{1}$.

The embedding of $C_{0}$ into $X$ endows $C_{0}$ with a complex structure. And, because $C_{0}$ has finitely many annular ends, $C_{0}$ is complex analytically equivalent to the complement of a finite number of points in a compact complex curve. This compact curve is the curve $\Sigma$ for Proposition 6.1 .

As for the map $\varphi$, consider that the embedding into $X$ of $\Sigma$ minus these finite points is a pseudo-holomorphic map which extends as a continuous map from the whole of $\Sigma$ into $X$. The latter is the map $\varphi$. Standard elliptic theory can be invoked 
to prove that $\varphi$ is a smooth map and is everywhere pseudo-holomorphic. (One need not refer to the removable singularities theorem for pseudo-holomorphic maps [SU] (see, e.g., [MS, Section 4.2]) which also asserts that $\varphi$ is pseudo-holomorphic on the whole of $\Sigma$. Indeed, the non-classical part of the removable singularities theorem is the one that establishes $\varphi$ 's continuity.)

\section{Constraints ON Symplectic 4-MANifoldS}

The purpose of this section is to discuss the theorems of the introductory section. Aside from Theorem 1.3, the principal player in these discussions is the genericity result which is quoted below as Proposition 7.1. The first two subsections of this section discuss Proposition 7.1; and the remaining subsections discuss the theorems of the Introduction.

(a) Regularity for generic $J$. For applications to the proofs of the assertions in the Introduction, it proves useful to have some a priori regularity results for the maps $\varphi$ which arise in Proposition 6.1. The proposition below serves this purpose by analyzing the possibilities for $\varphi$ for a suitably generic almost complex structure (though still one which is compatible with the given symplectic form $\omega$.) In this regard, remember that an almost complex $J$ is $\omega$-compatible when $\omega(\cdot, J(\cdot))$ defines a Riemannian metric on $X$. The form $\omega$ is self-dual for such a metric with length $\sqrt{2}$. Conversely, if $g$ is a metric for which $\omega$ is self-dual with length $\sqrt{2}$, then the almost complex structure defined by $g$ and $\omega$ is $\omega$-compatible.

The genericity results stated in Proposition 7.1 are probably known to pseudoholomorphic curve experts (see [Rua] and [MS]); however, the author has found no statement in the literature which summarizes the situation.

The proposition below requires a four part digression to introduce some terminology. Part 1 of the digression introduces the Fréchet space $\mathcal{C}_{\omega}$ of smooth almost complex structures which are $\omega$-compatible. For part 2 of the digression, let $e \in H^{2}(X ; \mathbb{Z})$ be a non-zero class and let $d \equiv-c \cdot e+e \cdot e$, where $c \equiv c_{1}(K)$ and where $\cdot$ is the cup product pairing. If $d \geq 0$, let $\Omega \subset X$ be a set of $d / 2$ distinct points. If $d \leq 0$, set $\Omega \equiv \varnothing$. Given $J \in \mathcal{C}_{\omega}$, use $\mathfrak{m}(e)$ to denote the space of pairs $(\varphi, \Sigma)$, where

(1) $\Sigma$ is a compact, complex curve.

(2) $\varphi: \Sigma \rightarrow X$ is a pseudo-holomorphic map which is not constant on any component of $\Sigma$.

(3) $\varphi_{*}[\Sigma]$ is Poincaré dual to $e$.

(4) $\Omega \subset \varphi(\Sigma)$.

(With regard to (7.1)(3), remark that complex curves have canonical orientations.)

For part 3 of the digression, suppose that $\Sigma$ is a compact, complex curve and that $\varphi: \Sigma \rightarrow X$ is a pseudo-holomorphic map. Say that $\varphi$ has multiplicity one if $\varphi$ does not factor as $\varphi^{\prime} \circ \psi$, where $\psi$ is a holomorphic map of $\Sigma$ to a complex curve $\Sigma^{\prime}$ with $\psi_{*}[\Sigma] \neq\left[\Sigma^{\prime}\right]$, and where $\varphi^{\prime}: \Sigma^{\prime} \rightarrow X$ is a pseudo-holomorphic map.

Part 4 introduces the notion of Baire subset of a complete metric space. This is, by definition, a countable intersection of open, dense sets. In particular, such a subset is dense. (And, any countable intersection of such sets is also Baire.)

Here is the regularity theorem: 
Proposition 7.1. Let $X$ be a compact, oriented 4-manifold with symplectic form $\omega$. There is a Baire set in $\mathcal{C}_{\omega}$ whose members have the following properties: First, fix a non-zero class $e \in H^{2}(X, \mathbb{Z})$ and set $d \equiv-c \cdot e+e \cdot e$. Then

(A) Suppose that $\mathfrak{m}(e)$ contains $(\varphi, \Sigma)$ such that $\varphi$ has multiplicity 1 on the union $S \subset \Sigma$ of those genus zero components whose fundamental classes have $\varphi_{*}$-images with negative square. Then, the following is true:

(1) If $d<0$, then $\mathfrak{m}(e)=\varnothing$.

(2) When $(\varphi, \Sigma) \in \mathfrak{m}(e)$, then $\varphi$ embeds all genus $g \neq 1$ components of $\Sigma$.

(3) When $(\varphi, \Sigma) \in \mathfrak{m}(e), \varphi$ on any genus 1 component of $\Sigma$ is a covering map onto some embedded, pseudo-holomorphic torus in $X$.

(4) When $(\varphi, \Sigma) \in \mathfrak{m}(e)$, the images of the components of $\Sigma$ under $\varphi$ are disjoint.

(5) The set of images $\{\varphi(\Sigma):(\varphi, \Sigma) \in \mathfrak{m}(e)\}$ is finite.

(B) In general, let $S \subset \Sigma$ be as in (A). Then the image of $S$ under $\varphi$ is a disjoint union of embedded, pseudo-holomorphic spheres with self-intersection number -1 .

(b) Proof of Proposition 7.1. This subsection contains the proof of Proposition 7.1 .

There are two ingredients to the arguments. The first ingredient is the SardSmale theorem [SS] and transversality theory on Banach manifolds. The second ingredient is the adjunction formula. The latter gives the genus $g$ of an immersed (multiplicity 1) pseudo-holomorphic curve in terms of the degree of the normal bundle of the curve and the cohomology class $e$ of its Poincaré dual. The simplest case is when $\Sigma$ is a compact, connected, complex curve and $\varphi: \Sigma \rightarrow X$ is an embedding. Then

$$
g=1+\frac{1}{2}(c \cdot e+e \cdot e)
$$

where $c$ is the first Chern class of the canonical bundle $K$. In the case where $\varphi$ is an immersion with $n$ double points,

$$
g=1+\frac{1}{2}(c \cdot e+e \cdot e-2 n) .
$$

(Note that all of the double points of an immersed pseudo-holomorphic curve have positive local intersection number. Thus, $e \cdot e-2 n$ is the degree of the normal bundle of $\varphi(\Sigma)$.) The numerology also involves an integer

$$
d \equiv-c \cdot e+e \cdot e-2 \cdot n .
$$

The proof of Proposition 7.1 is established in a series of steps. For this purpose, introduce $H(e)$ to denote a component of the space of $(\varphi, \Sigma)$ which obey $(7.1)(1)$ (7.1)(3).

Step 1. Consider first the case where $H(e)$ contains some pair $(\varphi, \Sigma)$ where $\Sigma$ is connected and where $\varphi$ has multiplicity one. If $\Sigma$ has genus zero, arguments in [Rua] establish a Baire subset $\mathcal{C}^{1} \subset \mathcal{C}_{\omega}$ with the property that $H(e)=\varnothing$ unless $d \geq 0$. By the adjunction formula, this requires $d=(1-2 n+e \cdot e) \geq 0$, which requires $e \cdot e \geq-1$. Likewise, the arguments in [Rua] establish a path connected, Baire subset $\mathcal{C}^{1} \subset \mathcal{C}_{\omega}$ for which $H(e)=\varnothing$ unless $d \geq 0$ and $e \cdot e \geq g-1+2 n$. (Thus, genus positive curves have non-negative self-intersections.)

Step 2. Suppose that $H(e)$ contains a pair $(\varphi, \Sigma)$ where $\Sigma$ has just one component and $\varphi$ has multiplicity 1 . The arguments in [Rua] prove that there is a Baire subset $\mathcal{C}^{2} \subset \mathcal{C}^{1}$ for which $H(e)$ is a stratified space whose smooth strata are the inverse 
images of the smooth strata of the tautological map to the moduli space of complex structures on $\Sigma$. The dimension of the top strata is equal to $d$ in the case where $g \geq 2$, and it equals $d+2$ and $d+6$ when $g=1$ and 0 , respectively. (In these last two cases, there are a 2 and 6 dimensional family of degree one holomorphic maps from $\Sigma$ to itself.)

Step 3. Suppose that $H(e)$ contains a pair $(\varphi, \Sigma)$ where $\Sigma$ has one component and $\varphi$ has multiplicity 1. Let $H(e)_{c} \subset H(e)$ denote the set of $(\varphi, \Sigma)$ for which $\varphi$ is not an immersion. The arguments in Chapter 6 of [MS] generalize readily to the case of genus $g>0$ surfaces to prove that there is a Baire subset $\mathcal{C}^{3} \subset \mathcal{C}^{2}$ for which the set $H(e)_{c} \subset H(e)$ is a subvariety which intersects each stratum transversely as a codimension 2 subvariety. Note that this step plus the previous two imply Assertion B.

Step 4. Suppose that $H(e)$ contains a pair $(\varphi, \Sigma)$ where $\Sigma$ has one component and $\varphi$ has multiplicity 1 . Let $\Omega \subset X$ be a set of some number $m>0$ distinct points. The arguments in [Rua] prove that there is a Baire subset $\mathcal{C}^{4} \subset \mathcal{C}^{3}$ for which the subspace $H(e ; \Omega) \subset H(e)$ of pairs $(\varphi, \Sigma)$ with $\Omega \subset \varphi(\Sigma)$ is a subvariety which intersects each stratum and each stratum of $H(e)_{c}$ transversely as a codimension $m$ submanifold.

Step 5 . Let $e, e^{\prime} \in H^{2}(X ; \mathbb{Z})$ be classes and suppose that corresponding spaces $H(e)$ and $H\left(e^{\prime}\right)$ both contain pairs $(\varphi, \Sigma)$ where $\Sigma$ is connected and $\varphi$ has multiplicity 1. The arguments in Chapter 6 of $[\mathrm{MS}]$ generalize readily to establish a Baire subset $\mathcal{C}^{5} \subset \mathcal{C}^{3}$ for which the subspace of $\left((\varphi, \Sigma),\left(\varphi^{\prime}, \Sigma^{\prime}\right)\right) \in H(e) \times H\left(e^{\prime}\right)$ which do not intersect transversely at regular points of both $\varphi$ and $\varphi^{\prime}$ is a codimension 2 subvariety of $H(e) \times H\left(e^{\prime}\right)$. Furthermore, if, say, finite subsets $\Omega, \Omega^{\prime} \subset X$ have been a priori specified, then this variety in $H(e) \times H\left(e^{\prime}\right)$ can be assumed to intersect the product of any of the previously specified strata transversely as a codimension 2 subvariety.

(Remember that the local intersection number of two pseudo-holomorphic curves at a transversal intersection point equals 1.)

Step 6. Now, take the almost complex structure from $\mathcal{C}^{2}$. Let $(\varphi, \Sigma) \in H(e)$ have the property that each 2 -sphere component is mapped with multiplicity 1 . The components of $\Sigma$ form a set $\left\{\Sigma_{i}\right\}$, and suppose that $\left.\varphi\right|_{\Sigma_{i}}=\varphi_{i} \circ \psi_{i}$, where $\psi_{i}$ is a degree $m_{i} \geq 1$ holomorphic map from $\Sigma_{i}$ to a compact complex curve $\Sigma_{i}^{\prime}$, and where $\varphi_{i}$ is a multiplicity one, pseudo-holomorphic map from $\Sigma_{i}^{\prime}$ into $X$. (If $m_{i}=1$, one can assume that $\Sigma_{i}^{\prime}=\Sigma_{i}$ and $\psi_{i}$ is the identity map.)

Write the Poincaré dual to $\varphi_{*}\left[\Sigma_{i}\right]$ as $m_{i} \cdot e_{i}$. Thus, $e=\sum_{i} m_{i} \cdot e_{i}$. Each pair $\left(\varphi_{i}, \Sigma_{i}\right)$ is in the corresponding $H\left(e_{i}\right)$, a stratified space of dimension $d_{i} \equiv$ $-c \cdot e_{i}+e_{i} \cdot e_{i}-2 n_{i}+\kappa_{i}$, where $n_{i}$ is the number of double points for the generic pair in $H\left(e_{i}\right)$ and where $\kappa_{i}=0,2$ or 6 depending on whether the genus of $\Sigma_{i}$ is greater than 1,1 or 0 .

When $i \neq j$, let $n_{i j}$ denote the intersection number of $\Sigma_{i}$ with $\Sigma_{j}$. Note that this is a priori non-negative.

Let $d \equiv-c \cdot e+e \cdot e$. Note that

$$
d=\sum_{i} m_{i} \cdot d_{i}+2 \cdot \sum_{i} n_{i}+\sum_{i}\left(m_{i}^{2}-m_{i}\right) e_{i} \cdot e_{i}+\sum_{i \neq j} n_{i j}
$$


is a sum of non-negative terms. In particular,

$$
d \geq \sum_{i} d_{i}
$$

with equality if and only if

(1) For all $i, m_{i}=1$ unless $d_{i}=e_{i} \cdot e_{i}=0$.

(2) For all $i, n_{i}=0$.

(3) For all $i \neq j, n_{i j}=0$.

This last equation with the previous steps (especially Step 4) establishes the first four parts of assertion (A) of Proposition 7.1.

Step 7. The fifth part of assertion (A) is proved in [Rua] except in the case where $\mathfrak{m}(e)$ contains a pair $(\varphi, \Sigma)$ where $\Sigma$ contains a genus 0 component for which the corresponding $m_{i}$ is greater than 1 and the corresponding $e_{i}$ obeys $c \cdot e_{i}=e_{i} \cdot e_{i}=0$. To make the proof for this case, it is sufficient to assume that $m(e)$ contains a pair $(\varphi, \Sigma)$ where $\Sigma$ is a torus which is mapped with multiplicity $m$ by $\varphi$ onto an embedded, pseudo-holomorphic torus $\Sigma^{\prime} \subset X$. Note that the map $\varphi$ must be, a priori, an $m$-fold covering map.

It follows from the preceding steps that there are no pseudo-holomorphic tori $\Sigma^{\prime \prime} \subset X$ which are homologous to $\Sigma^{\prime}$ and close to $\Sigma^{\prime}$ in $X$. Thus, the only way the assertion in question can fail is if there is a sequence $\left\{\Sigma_{\nu}\right\}$ of embedded, pseudoholomorphic tori in $X$ with the following properties:

(1) $\left[\Sigma_{\nu}\right]=n \cdot\left[\Sigma^{\prime}\right]$ for some $n>1$ which divides $m$.

(2) $\lim _{\nu \rightarrow \infty}\left\{\rho_{\nu} \equiv \sup \left\{\operatorname{dist}\left(x, \Sigma^{\prime}\right): x \in \Sigma_{\nu}\right\}\right\}=0$.

Note that (7.8)(1) implies that no $\Sigma_{\nu}$ can intersect $\Sigma^{\prime}$ because the self-intersection number of $\Sigma^{\prime}$ is, by assumption, zero. And, (7.8)(2) asserts that for $\nu$ large, $\Sigma_{v}$ lies in any a priori specified tubular neighborhood of $\Sigma^{\prime}$ as a pseudo-holomorphic curve.

With the preceding understood, remark that the exponential map for the Riemannian metric identifies a tubular neighborhood of $\Sigma^{\prime}$ with a disk bundle in the normal bundle, $N$, to $\Sigma^{\prime}$. With this understood, one can identify $\Sigma_{\nu}$ (for $\nu$ large) with an embedded torus in the normal bundle to $\Sigma^{\prime}$; this will, henceforth, be done. With this understood, rescale the fibers of $N$ by a $\nu$ dependent constant so that the rescaled $\Sigma_{\nu}$, call it $\Sigma_{\nu}^{\prime}$, is such that the point of maximum distance from the zero section has distance 1 . Note that the rescaling produces, for large $\nu$, a new almost complex structure, $J_{\nu}$, on the radius 2 disk bundle.

The sequence $\left\{J_{\nu}\right\}$ of almost complex structures will converge to an almost complex structure, $J_{0}$, which extends over the total space of $N$ as a homogeneous almost complex structure. That is, $J_{0}$ is invariant under the group of diffeomorphisms of $N$ which multiply the fibers by a non-zero real number. Meanwhile, the sequence $\left\{\Sigma_{\nu}^{\prime}\right\}$ will have a subsequence which converges to a $J_{0}$-pseudo-holomorphic torus $\Sigma^{1}$ in the normal bundle to $\Sigma^{\prime}$ with the property that the projection to $\Sigma^{\prime}$ is an $n$-fold covering map. With the preceding understood, the relevant question is: Which homogeneous almost complex structures admit such a $\Sigma^{1}$ ?

To analyze homogeneous almost complex structures, remark that one can choose fiber coordinates $\xi$ for $N$ so that the fibers of $N$ are $J_{0}$-pseudo-holomorphic, as is 
the zero section $\xi \equiv 0$. One can even choose a globally defined complex coordinate $\xi$ for the fiber of $N$ so that $J_{0}$ is as follows:

$$
J_{0} \cdot d \xi=i \cdot d \xi+B_{+} \xi+B_{-} \xi^{*}
$$

where $B_{ \pm}$are complex valued, $(0,1)$-forms on $\Sigma^{\prime}$. (Remember that $N$ is a topologically trivial vector bundle since $\Sigma^{\prime}$ has square zero.) Note that this $J_{0}$ is integrable where $B_{-}$vanishes.

With $J_{0}$ understood, remark that $\Sigma^{1}$ can be viewed as a section $s$ of a certain flat (hence holomorphic), rank $n$, complex vector bundle over $\Sigma^{\prime}$. The section $s$ must also obey the equation:

$$
\partial^{*} s+\frac{1}{2 i}\left(B_{+} s+B_{-} s^{*}\right)=0 .
$$

The task now is to prove that (7.10) has no solutions for a suitably generic choice of data $B_{ \pm}$. To prove that such is the case, fix sections $B_{+}^{0}, B_{-}^{0}$ and $b$ of $T^{0,1}$ with $b$ nowhere vanishing. (Because $\Sigma^{\prime}$ is a torus, $T^{0,1}$ is topologically trivial.) Then, consider (7.10) with $B_{+} \equiv B_{+}^{0}$ and with $B_{-} \equiv B_{-}^{0}+t \cdot b$ with $t \in \mathbb{C}$ allowed to vary. The first observation (using the Weitzenböch formula for (7.10)) is that there are no solutions when $|t|$ is sufficiently large. (This is because $b$ is nowhere vanishing.) Meanwhile, simple arguments from analytic perturbation theory prove that the set of $t$ for which (7.10) has a solution is either the whole of $\mathbb{C}$ or else a discrete set. Here is the point: For given $t \in \mathbb{C}$, let $L_{t}$ denote the operator in (7.10). Now, fix $t_{0} \in \mathbb{C}$. Perturbation theory produces a neighborhood $D \subset \mathbb{C}$ of $t_{0}$ and, when $t \in D$, a matrix $M_{t}$ from the (finite dimensional) kernel of $L_{t_{0}}$ to the cokernel of $L_{t_{0}}$ with the following properties: $\operatorname{First}, \operatorname{kernel}\left(M_{t}\right)$ and $\operatorname{kernel}\left(L_{t}\right)$ are naturally identified by $L^{2}$-orthogonal projection of the latter onto kernel $\left(L_{t_{0}}\right)$. Second, the matrix $M_{t}$ can be constructed to vary complex analytically with $t \in D$. Now, the index of $L_{t_{0}}$ is zero, so the kernel of $M_{t}$ is non-trivial if and only if the determinant of $M_{t}$ is zero. But, $M_{t}$ varies analytically with $t \in D$, so its determinant defines an analytic function on $D$ and so vanishes discretely or completely.

(c) Proofs for minimal $X$ of Theorems 0.1 and 0.2. In discussing Theorems 0.1 and 0.2 , it is convenient to first establish both in the case where $X$ is minimal in the following sense:

$$
X \text { has no embedded 2-spheres with self-intersection number }-1 \text {. }
$$

Consider first the proof of Theorem 0.1 with this assumption:

Proof of Theorem 0.1. First of all, take one of Proposition 7.1's generic, $\omega$-compatible almost complex structures, and use this almost complex structure and $\omega$ to define the Riemannian metric on $X$. As $X$ has $b_{2}^{+}>1$, the Seiberg-Witten invariants are well defined; in particular, they are independent of the perturbation $\mu$ in (1.8). Thus, if the Seiberg-Witten invariant of $e$ is non-zero, then (1.20) has a solution for the Spin ${ }^{\mathbb{C}}$ structure in (1.14) for every value of $r$. With this understood, invoke Theorem 1.3 and Proposition 7.1. Theorem 1.3 finds a smooth, compact, complex curve $\Sigma$ and a pseudo-holomorphic map $\varphi: \Sigma \rightarrow X$ which pushes the fundamental class of $\Sigma$ forward as the Poincaré dual of $e$. Proposition 7.1 then describes the possibilities for such a map. In particular, $\varphi$ has to be an embedding except possibly on genus zero or genus 1 components of $\Sigma$. The minimality assumption and Proposition 7.1 imply that $\Sigma$ has no genus zero components. If $\Sigma$ has a genus one component, then $\varphi$ will map this component as a multiple cover onto a 
pseudo-holomorphic submanifold $\Sigma_{1} \subset X$ which itself has genus 1. Proposition 7.1 plus the adjunction formula (7.2) imply that $\Sigma_{1}$ has trivial normal bundle. Thus, for $n \geq 1$, the class $n \cdot\left[\Sigma_{1}\right]$ can be represented by a symplectically embedded torus in the normal bundle to $\Sigma_{1}$.

Proof of Theorem 0.2. Theorem 0.1's proof finds a set of $\omega$-compatible, almost complex structures with the property that:

$$
\text { A non-zero class } e \in H^{2}(X ; \mathbb{Z}) \text { with } \mathrm{SW}(e) \neq 0 \text { is Poincaré dual to the }
$$

fundamental class of a pseudo-holomorphic submanifold $\Sigma \subset X$.

In the discussions below all almost complex structures under consideration are assumed to satisfy (7.12).

Assertion (1). This assertion follows from Theorem 0.1 and the main theorem in [T2] which asserts that $\mathrm{SW}\left(c_{1}(K)\right)$ is equal to \pm 1 . (Also, $\mathrm{SW}(0)= \pm 1$.)

The second assertion is inoperative for a minimal $X$.

Assertion (3). By (7.10) and the main theorem in [T2], there is an embedded, pseudo-holomorphic submanifold $\Sigma$ whose fundamental class is Poincaré dual to $c_{1}(K)$. Let $\left\{\Sigma_{i}\right\}$ denote the components of $\Sigma$. It follows from Proposition 7.1 and the adjunction formula that the only components of $\Sigma$ with negative self-intersection number are spheres of self-intersection -1 . Thus, $c_{1}(K)$ has positive square.

Assertion (4). This assertion follows from assertion (3) and the fact that $d$ in (1.9) is equal to zero for the canonical $\operatorname{Spin}^{\mathbb{C}}$ structure. (Note that $L=K^{-1}$ in this case.)

Assertion (5). Note first that if $c_{1}(K)$ has zero square, then (7.12), the adjunction formula (7.2) and Proposition 7.1 imply that $c_{1}(K)$ is Poincaré dual to a disjoint union of embedded tori.

Now, let $e \in H^{2}(X ; \mathbb{Z})$ be a class with $\mathrm{SW}(e) \neq 0$. The case for $e=0$ is automatically true, so assume that $e$ is not trivial. Note that (7.12) and Proposition 7.1 insure that $e \cdot e \geq 0$ since $X$ is assumed to be minimal.

To go farther, it is necessary to digress momentarily to introduce a generalized adjunction formula from [KM1] and [MST]. Here is this formula in the symplectic context:

The Generalized Adjunction Formula. Let $X$ be a compact, oriented symplectic manifold with $b_{2}^{+}>1$. Fix a class $k \in H^{2}(X ; \mathbb{Z})$ with $\mathrm{SW}(k) \neq 0$; and fix a class $s \in H^{2}(X ; \mathbb{Z})$ with non-negative square. Let $g_{s}$ denote the smallest integer that is the genus of an embedded surface whose fundamental class is Poincaré dual to $s$. Then

$$
g_{s} \geq 1+\frac{1}{2}\left(\left|-c_{1}(K) \cdot s+2 k \cdot s\right|+s \cdot s\right) .
$$

(Compare with the celebrated $\mathrm{SU}(2)$-gauge theory version of this inequality in [KM2].)

End the digression.

Consider the preceding adjunction formula with $k=e$ and with $s$ equal to the Poincare dual to the fundamental class of any of the components of the pseudoholomorphic submanifold which is Poincaré dual to $c_{1}(K)$. (These are supplied courtesy of (7.12).) Since each of the aforementioned components is a torus with 
self-intersection zero, the inequality in $(7.13)$ can be satisfied only if $c_{1}(K) \cdot e=0$. In fact, since embedded pseudo-holomorphic curves intersect positively, (7.13) implies that the components of the pseudo-holomorphic curve which represents $e$ are all disjoint from those that represent $c_{1}(K)$.

To complete the argument, return again to (7.13) but this time take $s$ and $k$ both equal to $e$. The resulting inequality is compatible with the adjunction formula in (7.2) only if $e \cdot e=0$. In this case, (7.12), the minimality of $X$ and Proposition 7.1 imply that each component of the pseudo-holomorphic submanifold which is Poincaré dual to $e$ must be a torus with zero self-intersection number.

Assertion (6). This assertion is also a consequence of (7.13). To start, use (7.12) to obtain a pseudo-holomorphic submanifold of $X$ whose fundamental class is Poincaré dual to $e$. Once again, the minimality of $X$ and Proposition 7.1 imply that $e \cdot e \geq 0$. With this understood, suppose that $d \equiv-c_{1}(K) \cdot e+e \cdot e>0$. (Note that $\mathrm{SW}(e) \equiv 0$ by definition if $d<0$.) Positivity of $d$ implies that $e \cdot e>c_{1}(K) \cdot e$ and this makes (7.12) incompatible with (7.2) in the case where $k=e$ and $s=e$.

(d) Proof of Theorems 0.1 and 0.2 when $X$ is not minimal. Consider first the case for Theorem 0.1 .

Proof of Theorem 0.1 . The only new issue is that $\Sigma$ can have one or more 2 -sphere components. In this case, Proposition 7.1 asserts that with a generic choice of $\omega$ compatible almost complex structure, $\varphi$ maps $S$ onto a union of disjoint, embedded, pseudo-holomorphic 2 -spheres in $X$, each with square -1 . There is nothing more to say if $\varphi$ is an embedding, and the claim is that such is always the case.

To prove the claim assume to the contrary that $\varphi$ on $S$ is not 1-1 onto some 2sphere component $\Sigma_{0}$ of its image. To obtain a contradiction, note first that $\Sigma_{0}$ is, in any case, pseudo-holomorphic with square -1 . This means that $X$ is a symplectic blow up. That is, $X=Y=\underline{\mathbb{C P}}^{2}$, and $Y$ is a symplectic manifold. Furthermore, let $e_{0}$ denote the Poincaré dual to the fundamental class of $\Sigma_{0}$. Since $\varphi$ multiply covers $\Sigma_{0}$, there is an integer $m>1$ such that $e_{1} \equiv e-m \cdot e_{0}$ is Poincaré dual to a sum of positive multiples of the Poincare duals of the components of $\Sigma_{1} \equiv \Sigma-\Sigma_{0}$. Note that $\Sigma_{1}$ can be assumed to sit in $Y$, and by Poincaré duality, this identifies $e_{1}$ with a non-zero class $e_{1}^{\prime} \in H^{2}(Y ; \mathbb{Z})$.

A digression is now required to point out that there is a blow-up formula which relates the Seiberg-Witten invariants of $Y$ to those of $X$. (This was proved independently by Kronheimer and Mrowka [Mr] and by Fintushel and Stern [FS].) In particular, the blow-up formula has the following relevant consequences:

(1) Define simple type as in assertion (6) of Theorem 0.2. If $Y$ has simple type, then so does $X$.

(2) $\operatorname{SW}(e)=0$ if and only if $\operatorname{SW}\left(e_{1}^{\prime}\right)=0$.

End the digression.

To use (7.14), remark first that (7.14)(1) implies that any symplectic manifold has simple type. That is, (7.14)(1) implies assertion (6) of Theorem 0.2 in the general case. Indeed, the proof for minimal $Y$ is given above, and any symplectic $Y$ can be obtained from a minimal one by a sequence of symplectic blowups.

With the preceding understood, note that as $m>1$, the integer $d \equiv-c_{1}(K) \cdot e$ $+e \cdot e$ will be negative unless $d_{1} \equiv-c_{1}(K) \cdot e_{1}+e_{1} \cdot e_{1}>0$. And, this $d_{1}$ is the dimension of the relevant Seiberg-Witten moduli space for computing $\mathrm{SW}\left(e_{1}^{\prime}\right)$. 
Thus, in the case where $m>1$, one finds that $\mathrm{SW}(e) \neq 0$ requires that $Y$ not have simple type as described in assertion (6) of Theorem 0.2. This contradiction gives the initial claim and Theorem 0.1 in the non-minimal case.

Proof of Theorem 0.2. Only assertions (1) and (2) remain to be proved. (Assertion (6) was proved above.) As in the minimal case, the first assertion follows from Theorem 0.1 and the main result in [T2].

Consider assertion (2): To begin, remember that [T2] asserts in part that SW(0) and $\mathrm{SW}\left(c_{1}(K)\right)$ are both \pm 1 . Using the sphere of square -1 , one can construct a diffeomorphism of $X$ which acts on $H^{2}(X ; \mathbb{Z})$ as reflection in the hyperplane which is orthogonal to $e$. The author was reminded by Daniel Ruberman that $c_{1}(K) \cdot e \neq 0$ because $c_{1}(K)$ is a characteristic class. Thus, $c_{1}(K)$ is not fixed by the diffeomorphism. According to Proposition 1.2, the Seiberg-Witten invariant as a map from Spin to $H^{2}(X ; \mathbb{Z})$ is diffeomorphism invariant. However, the identification in (0.2) is not diffeomorphism invariant. Sorting this out, one finds that $\mathrm{SW}(m \cdot e)= \pm 1$, where $m=-c_{1}(K) \cdot e$ and where SW is as in (0.3). Theorem 1.3 and Proposition 7.1 provide a Baire set of $\omega$-compatible, almost complex structures for which $e$ is represented as $s+t$, where $s$ is Poincaré dual to a pseudo-holomorphic sphere with self-intersection -1 and where $t$ is Poincaré dual to the fundamental class of a symplectic submanifold which is disjoint from the 2 -sphere for $s$. It remains now to prove that $t \equiv 0$.

To begin, remark that because $s$ and $t$ are Poincaré dual to disjoint subsets in $X$, one has $t \cdot t=0$ because $e \cdot e=-1=s \cdot s$.

The next step argues that $m \equiv-c_{1}(K) \cdot e=1$. To prove this claim, consider first the orbit $\mathcal{O} \subset H_{2}(X ; \mathbb{Z})$ of $-c_{1}(K)$ under the action of the group $\mathcal{G}$ which is generated by the reflections in the planes orthogonal to $s$ and to $e$. Note that every element in $\mathcal{G}$ is generated by a diffeomorphism of $X$. Thus, if $-c_{1}(K)+2 \tau \in \mathcal{O}$, then $\operatorname{SW}(\tau)= \pm 1$. With the preceding understood, look now at the element $T \in \mathcal{G}$ which is given by $T(\tau) \equiv \tau+2[\tau \cdot s-\tau \cdot e] s+2(\tau \cdot e) t$. (To obtain $T$, first reflect through the plane orthogonal to $e$ and then reflect through the plane orthogonal to $s$.) Since the Seiberg-Witten invariant is non-zero on only finitely many classes [W], the set of classes $\left\{T^{n}\left(-c_{1}(K)\right)\right\}_{n \geq 1}$ must be finite. The latter requirement will be seen to require that $m=1$.

To analyze $c^{n} \equiv T^{n}\left(c_{1}(K)\right)$, observe that

$$
\begin{aligned}
& \text { (1) } c^{n} \cdot e=c^{n-1} \cdot e+2 \cdot\left(c^{n-1} \cdot e-c^{n-1} \cdot s\right) . \\
& \text { (2) } c^{n} \cdot s-c^{n} \cdot e=c^{n-1} \cdot e-c^{n-1} \cdot s .
\end{aligned}
$$

Thus, the coefficient of $t$ in $c^{n}$ will grow in absolute value with $n$ unless $c_{1}(K) \cdot e=$ $c_{1}(K) \cdot s$. As the latter is equal to -1 , the claim is proved.

The third step asserts that $-c_{1}(K)-2 \cdot t$ is in $\mathcal{O}$. Given this claim, it follows that $t=0$ since both $\pm t$ cannot be Poincaré dual to fundamental classes of symplectic submanifolds. (If $\tau$ is the Poincaré dual to the fundamental class of a symplectic submanifold, then $[\omega] \cdot \tau>0$.) To prove the assertion, observe first that reflection in the plane orthogonal to $s$ sends $-c_{1}(K)$ to $-c_{1}(K)+2 s$. Then, reflection in the plan orthogonal to $e=s+t$ sends the latter class to $-c_{1}(K)-2 t$ (since $t \cdot c_{1}(K)=0$ because $\left.c_{1}(K) \cdot e=1\right)$.

(e) Proof of Theorem 0.3. Remember that $\mathbb{C P}^{2}$ has a metric with positive scalar curvature. This implies that the Seiberg-Witten invariants as computed using (1.7) 
all vanish. Furthermore, since $\mathbb{C P}^{2}$ has positive definite intersection form, the invariants as computed using (1.7) vanish for any choice of metric. Now, let $\omega$ be a symplectic form for $\mathbb{C P}^{2}$ and choose an $\omega$-compatible almost complex structure $J$ from the Baire set from Proposition 7.1.

The key observation is that for larger $r$ in (1.20), the invariants will not vanish. In particular, let $e \in H^{2}\left(\mathbb{C P}^{2} ; \mathbb{Z}\right)$ denote the generator which has $e \cdot[\omega]>0$. (Note that [T5] proves that $c_{1}(K) \cdot[\omega]$ is negative.) Then argue as in [T5] that for $r>r_{0} \equiv 2 \pi e \cdot[\omega]$, the invariant SW as computed by solutions to (1.20) has $\mathrm{SW}(e) \neq 0$. (At $r=r_{0}$, the moduli space of solutions consists solely of $(A,(0,0))$, where $A$ has curvature equal to $r_{0} \cdot i \cdot \omega$. For $r$ slightly greater than $r_{0}$, perturbation theory identifies the moduli space of solutions to (1.20) as a copy of $\mathbb{C P}^{2}$, and the invariant $\mathrm{SW}(e)$ can be calculated to equal 1.) There are no other wall crossings for $r>r_{0}$. With this understood, apply Theorem 1.3 and Proposition 7.1 to find an embedded, pseudo-holomorphic 2-sphere for the almost complex structure $J$. Given such a 2-sphere, a theorem of Gromov in $[\mathrm{Gr}]$ asserts that there is a diffeomorphism of $\mathbb{C P}^{2}$ which takes $\omega$ to a multiple of the standard Kähler form.

\section{REFERENCES}

[Ar] N. Aronszajn, A unique continuation theorem for elliptic differential equations or inequalities of the second order, J. Math. Pures Appl. 36 (1957), 235-249. MR 19:1056c

[Au] T. Aubin, Nonlinear Analysis on Manifolds, Monge-Ampere Equations, Springer-Verlag, New York, 1982. MR 85j:58002

[Ch] S. Chang, Two dimensional area minimizing integral currents are classical minimal surfaces, JAMS 1 (1988), 699-778. MR 89i:49028

[Do] S. K. Donaldson, Lectures at MIT, September 1994.

[DN] A. Douglis and L. Nirenberg, Interior estimates for elliptic systems of partial differential equations, Comm. Pure Appl. Math. 8 (1955), 503-538. MR 17:743b

[Fe] H. Federer, Geometric Measure Theory, Springer-Verlag, New York, 1969. MR 41:1976

$[\mathrm{FF}]$ H. Federer and W. Fleming, Normal and integral currents, Ann. of Math. 72 (1960), 458-520. MR 23:A588

[FS] R. Fintushel and R. Stern, Immersed spheres in 4-manifolds and the immersed Thom Conjecture, preprint, 1995.

[FU] D. Freed and K. K. Uhlenbeck, Instantons and Four Manifolds, Springer-Verlag, New York, 1984. MR 86c:57031

[Gr] M. Gromov, Pseudo-holomorphic curves in symplectic manifolds, Invent. Math. 82 (1985), 307-347. MR 87j:53053

[JT] A. Jaffe and C. H. Taubes, Vortices and Monopoles, Birkhäuser, Boston, 1980. MR 82m:81051

[Ki] J. R. King, The currents defined by analytic varieties, Acta Math. 127 (1971), 185-220. MR 52:14359

[KM1] P. B. Kronheimer and T. S. Mrowka, The genus of embedded surfaces in the projective plane, Math. Res. Letters 1 (1994), 797-808. MR 96a:57073

[KM2] - Recurrence relations and asymptotics for four-manifold invariants, Bull. Amer. Math. Soc. 30 (1994), 215-221. MR 94k:57046

[La] H. B. Lawson, Minimal Varieties in Real and Complex Geometry, Sem. Math. Sup. Vol. 57, Presses Université de Montreal, 1974. MR 57:13798

[MS] D. McDuff and D. Salamon, J-Holomorphic Curves and Quantum Cohomology, preprint.

[MST] J. W. Morgan, Z. Szabó, and C. H. Taubes, A product formula for the Seiberg-Witten invariants and the generalized Thom conjecture, preprint, 1995.

[Mo] C. B. Morrey, Multiple integrals in the Calculus of Variations, Springer-Verlag, Berlin, 1966. MR 34:2380

[Mr] T. S. Mrowka, Lectures at Harvard University, Spring, 1995. 
[Rua] Y. Ruan, Symplectic topology and complex surfaces, in Geometry and Topology on Complex Manifolds, T. Mabuchi, J. Noguchi, T. Ochial, eds., World Scientific Publications, Singapore, 1994.

[Rut] H. Rutishauser, Uber die Folgen und Scharen von analytishen und meromorphen Funktionen mehrerer Variabeln, sowie von analytishen Abbildungen, Acta Math. 83 (1950), 249-325. MR 12:90f

[SU] J. Sacks and K. K. Uhlenbeck, The existence of minimal immersions of 2-spheres, Ann. of Math. 113 (1981), 1-24. MR 82f:58035

[SW1] N. Seiberg and E. Witten, Electro-magnetic duality, monopole condensation and confinement in $N=2$ supersymmetric Yang-Mills theory, Nucl. Phys. B426 (1994), 19-52. MR 95m:81202a; MR 95m:81202b

[SW2] _ Monopoles, duality and chiral symmetry breaking in $N=2$ supersymmetric $Q C D$, Nucl. Phys. B431 (1994), 485-550. MR 95m:81203

[SS] S. Smale, An infinite dimensional version of Sard's theorem, Amer. J. Math. 87 (1965), 861-866. MR 32:3067

[St] W. Stoll, The growth of the area of a trancendental analytic set. I, Math. Ann. 156 (1964), 47-78. MR 29:3670

[T1] C. H. Taubes, The Seiberg-Witten and the Gromov invariants, Math. Res. Letters 2 (1995), 221-238. MR 96a:57076

[T2] - The Seiberg-Witten invariants and symplectic forms, Math. Res. Letters 1 (1995), 809-822. MR 95j:57039

[T3] - On the equivalence of first and second order equations for gauge theories, Commun. Math. Phys. 75 (1980), 207-227. MR 83b:81098

[T4] Arbitrary n-vortex solutions to the first order Ginzburg-Landau equations, Commun. Math. Phys. 72 (1980), 277-292. MR 83c:81124

[T5] More constraints on symplectic manifolds from the Seiberg-Witten invariants, Math. Res. Letters 2 (1995), 9-13. MR 96a:57075

[W] E. Witten, Monopoles and 4-manifolds, Math. Res. Letters 1 (1994), 769-796. CMP 95:05

[Ye] R. Ye, Gromov's compactness theorem for pseudo-holomorphic curves, Trans. Amer. Math. Soc. 342 (1994), 671-694. MR 94f:58030

Department of Mathematics, Harvard university, Cambridge, Massachusetts 02138

E-mail address: chtaubes@math.harvard.edu 